\title{
The $\ell$-modular local Langlands correspondence and local constants
}

\author{
R. Kurinczuk*, N. Matringe ${ }^{\dagger}$
}

October 8, 2019

\begin{abstract}
Let $F$ be a non-archimedean local field of residual characteristic $p, \ell \neq p$ be a prime number, and $\mathrm{W}_{F}$ the Weil group of $F$. We classify equivalence classes of $\mathrm{W}_{F}$-semisimple Deligne $\ell$ modular representations of $\mathrm{W}_{F}$ in terms of irreducible $\ell$-modular representations of $\mathrm{W}_{F}$, and extend constructions of Artin-Deligne local constants to this setting. Finally, we define a variant of the $\ell$-modular local Langlands correspondence which satisfies a preservation of local constants statement for pairs of generic representations.
\end{abstract}

\section{Introduction}

Let $F$ denote a non-archimedean local field of residual cardinality $q$ and residual characteristic $p$. Let $\ell$ be a prime different to $p$. We consider only smooth representations of locally profinite

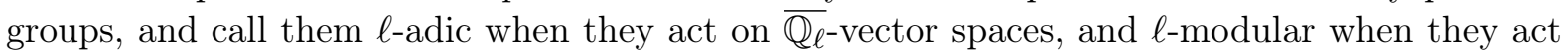
on $\overline{\mathbb{F}_{\ell}}$-vector spaces. Let $\mathrm{W}_{F}$ denote the Weil group of $F$.

The local Langlands correspondence LLC for $\mathrm{GL}_{n}(F)$ is a canonical bijection between the set of isomorphism classes of $\ell$-adic irreducible representations of $\mathrm{GL}_{n}(F)$ and the set of isomorphism classes of $\ell$-adic $n$-dimensional $\mathrm{W}_{F}$-semisimple Deligne representations, generalizing the Artin reciprocity map of local class field theory. An important property of LLC is that the RankinSelberg local constants of a pair of irreducible $\ell$-adic representations of $\mathrm{GL}_{n}(F)$ and $\mathrm{GL}_{m}(F)$, and the Artin-Deligne local constants of the corresponding tensor product of Deligne representations of $\mathrm{W}_{F}$ are equal, and moreover this condition characterizes LLC completely.

In [19], Vignéras develops the theory of $\ell$-modular representations of reductive $p$-adic groups. For general linear groups, this culminates in the $\ell$-modular local Langlands correspondence [24]: a bijection between the set of isomorphism classes of $\ell$-modular irreducible representations of $\mathrm{GL}_{n}(F)$ and the set of isomorphism classes of $\ell$-modular $n$-dimensional $\mathrm{W}_{F}$-semisimple Deligne representations with nilpotent Deligne operator. Vignéras characterizes her correspondence by compatibility with LLC and congruences, although not naively (see Section 6.2 where we recall Vignéras' results precisely).

\footnotetext{
${ }^{*}$ Robert Kurinczuk, Department of Mathematics, Imperial College London, SW7 2AZ. U.K. Email: robkurinczuk@gmail.com, Tel: +44(0)7921 221967

${ }^{\dagger}$ Nadir Matringe, Université de Poitiers, Laboratoire de Mathématiques et Applications, Téléport 2 - BP 30179, Boulevard Marie et Pierre Curie, 86962, Futuroscope Chasseneuil Cedex. France.

Email: Nadir.Matringe@math.univ-poitiers.fr
} 
The theory of Rankin-Selberg local constants of Jacquet, Piatetski-Shapiro and Shalika has a natural extension to $\ell$-modular generic representations of $\mathrm{GL}_{n}(F)$ and $\mathrm{GL}_{m}(F)$, 12. However, via the $\ell$-modular local Langlands correspondence these factors do not agree with the factors of Artin-Deligne.

In this work, we classify the $\ell$-modular indecomposable $\mathrm{W}_{F}$-semisimple Deligne representations, extend the definitions of Artin-Deligne factors to this setting, and define an $\ell$-modular local Langlands correspondence where in the generic case, the Rankin-Selberg factors of representations on one side equal the Artin-Deligne factors of the corresponding representations on the other.

We now recall our definitions and conventions, and state our results precisely. Let $R$ be an algebraically closed field of characteristic $\ell$ different from $p$. Let $\nu: \mathrm{W}_{F} \rightarrow R^{\times}$be the unique character trivial on the inertia subgroup of $\mathrm{W}_{F}$ and sending a geometric Frobenius element to $q^{-1}$. Fix a nontrivial character $\psi: F \rightarrow R^{\times}$.

A Deligne $R$-representation of $\mathrm{W}_{F}$ is a pair $(\Phi, U)$ with $\Phi$ a finite dimensional smooth representation of $\mathrm{W}_{F}$ acting on an $R$-vector space, and $U \in \operatorname{Hom}_{\mathrm{W}_{F}}(\nu \Phi, \Phi)$ the associated Deligne operator. We say a Deligne $R$-representation $(\Phi, U)$ of $\mathrm{W}_{F}$ is nilpotent if $U$ is a nilpotent endomorphism of the vector space of $\Phi$, and $\mathrm{W}_{F}$-semisimple if $\Phi$ is a semisimple representation of $\mathrm{W}_{F}$. We write $(\Phi, U)^{\vee}$ for the dual of $(\Phi, U)$, see Definition 4.3 . All Deligne $\overline{\mathbb{Q}_{\ell}}$-representations of $\mathrm{W}_{F}$ are nilpotent, however as we shall soon see this is not the case for Deligne $\overline{\mathbb{F}_{\ell}}$-representations.

We say that indecomposable Deligne $R$-representations $(\Phi, U),\left(\Phi^{\prime}, U^{\prime}\right)$ of $\mathrm{W}_{F}$ are equivalent, and write $(\Phi, U) \sim\left(\Phi^{\prime}, U^{\prime}\right)$, if there exists $\lambda \in R^{\times}$such that $(\Phi, \lambda U) \simeq\left(\Phi^{\prime}, U^{\prime}\right)$, and we extend this definition and notation to general Deligne $R$-representations of $\mathrm{W}_{F}$ thanks to the Krull-Schmidt theorem (see Definition 4.6 and Remark 4.7).

Before stating our classification results, we need to define two fundamental examples. For $\Psi$ an irreducible $R$-representation $\mathrm{W}_{F}$, we denote by $\mathbb{Z}_{\Psi}$ the set $\left\{\nu^{k} \Psi / \simeq, k \in \mathbb{Z}\right\}$ of isomorphism classes.

(1) Let $\Psi$ be an irreducible $\overline{\mathbb{F}_{\ell}}$-representation of $\mathrm{W}_{F}$, then there is a minimal positive integer $o(\Psi)$ such that we have an isomorphism $\nu^{o(\Psi)} \Psi$ to $\Psi$. Let $I$ be such an isomorphism. Define a Deligne $\overline{\mathbb{F}_{\ell}}$-representation

$$
\begin{aligned}
\mathcal{C}(\Psi, I) & =\left(\Phi(\Psi), \mathcal{C}_{I}\right) \\
\Phi(\Psi) & =\bigoplus_{k=0}^{o(\Psi)-1} \nu^{k} \Psi \\
\mathcal{C}_{I}\left(x_{0}, \ldots, x_{o(\Psi)-1}\right) & =\left(I\left(x_{o(\Psi)-1}\right), x_{0}, \ldots, x_{o(\Psi)-2}\right) .
\end{aligned}
$$

In Proposition 4.18, we show that $\mathcal{C}(\Psi, I)$ is irreducible. The equivalence class of $\mathcal{C}(\Psi, I)$ depends only on $\mathbb{Z}_{\Psi}$, and we denote it by $\mathcal{C}\left(\mathbb{Z}_{\Psi}\right)$.

(2) For a positive integer $r$, we define a $\mathrm{W}_{F}$-semisimple nilpotent Deligne $\overline{\mathbb{F}_{\ell}}$-representation

$$
\begin{aligned}
{[0, r-1] } & =(\Phi(r), N(r)) \\
\Phi(r) & =\bigoplus_{k=0}^{r-1} \nu^{k}, \\
N(r)\left(x_{0}, \ldots, x_{r-1}\right) & =\left(0, x_{0}, \ldots, x_{r-2}\right) .
\end{aligned}
$$

In Lemma 4.27, we show that $[0, r-1]$ is indecomposable. 
We prove the following classification:

Main Theorem 1 (Theorems 4.24 and 4.35). Let $(\Phi, U)$ be an indecomposable $\mathrm{W}_{F}$-semisimple Deligne $\overline{\mathbb{F}_{\ell}}$-representation.

(1) There exists an irreducible $\overline{\mathbb{F}_{\ell}}$-representation $\Psi$ of $\mathrm{W}_{F}$ and a positive integer $r$ such that either:

(a)

$$
(\Phi, U) \sim[0, r-1] \otimes \Psi
$$

(b) there exists an isomorphism $I: \nu^{o(\Psi)} \Psi \rightarrow \Psi$ such that

$$
(\Phi, U) \sim[0, r-1] \otimes \mathcal{C}(\Psi, I) .
$$

(2) Under the notation of the last part, $(\Phi, U)$ is irreducible if and only if $r=1$.

(3) In case (1a), the isomorphism class of $[0, r-1] \otimes \Psi$ coincides with its equivalence class, and determines the isomorphism class of $\Psi$ and $r$ uniquely.

(4) In case $(1 \mathrm{~b})$, the equivalence class of $[0, r-1] \otimes \mathcal{C}(\Psi, I)$ only depends on $r$ and $\mathbb{Z}_{\Psi}$, and we denote it by $[0, r-1] \otimes \mathcal{C}\left(\mathbb{Z}_{\Psi}\right)$. The equivalence class determines $\mathbb{Z}_{\Psi}$ and $r$ uniquely.

Let $(\Phi, U)$ be a $\mathrm{W}_{F}$-semisimple Deligne $R$-representation of $\mathrm{W}_{F}$. For an indeterminant $X$, we put

$$
L(X,(\Phi, U))=\operatorname{det}\left(\left.(\mathrm{Id}-X \Phi(\operatorname{Frob}))\right|_{\operatorname{Ker}(U)^{I_{F}}}\right)^{-1},
$$

it is an Euler factor. Using results of [8], for $\psi$ a nontrivial character of $F$, we associate to $(\Phi, U)$ a local $\gamma$-factor $\gamma(X,(\Phi, U), \psi)$, which does not see the operator $U$, and put

$$
\epsilon(X,(\Phi, U), \psi)=\gamma(X,(\Phi, U), \psi) \frac{L(X,(\Phi, U))}{L\left(q^{-1} X^{-1},(\Phi, U)^{\vee}\right)} .
$$

Of course, these definitions coincide with the standard ones when $R=\overline{\mathbb{Q}_{\ell}}$, as well as in the case when $R=\overline{\mathbb{F}_{\ell}}$ and $(\Phi, U)$ is of the form $(\Phi, 0)$, see Section 5 .

Following the $\overline{\mathbb{Q}_{\ell}}$-case, we wish to define Artin-Deligne local constants of pairs of $\mathrm{W}_{F}$-semisimple Deligne $\overline{\mathbb{F}_{\ell}}$-representations of $\mathrm{W}_{F}$ via their tensor product. However, there are two immediate obstacles:

(1) The tensor product of semisimple representations of $\mathrm{W}_{F}$ is not necessarily semisimple, see Example 3.11 (3).

(2) The tensor product does not preserve equivalence, see Example 4.11 .

Both of these problems have natural solutions and we define the semisimple tensor product $\otimes_{\mathrm{ss}}$ of equivalence classes of $\mathrm{W}_{F}$-semisimple Deligne $\overline{\mathbb{F}_{\ell}}$-representations of $\mathrm{W}_{F}$ in Section 4.4

Finally, we move on to our results on the $\ell$-modular local Langlands correspondence. We call an isomorphism class of $\mathrm{W}_{F}$-semisimple nilpotent Deligne $\overline{\mathbb{F}_{\ell}}$-representations a $\mathrm{V}$-parameter and, for a positive integer $n$, denote by $\mathrm{V}$ the bijection of [24]:

$$
\left\{\begin{array}{c}
\text { Irreducible } \ell \text {-modular representations } \\
\text { of } \mathrm{GL}_{n}(F) \text { up to isomorphism }
\end{array}\right\} \stackrel{\mathrm{V}}{\longrightarrow}\{\mathrm{V} \text {-parameters of dimension } n\}
$$


For $\psi$ a nontrivial character of $F$ and $\pi, \pi^{\prime}$ generic $\overline{\mathbb{F}_{\ell}}$-representations of $\mathrm{GL}_{n}(F), \mathrm{GL}_{m}(F)$ respectively we denote by

$$
L\left(X, \pi, \pi^{\prime}\right), \quad \epsilon\left(X, \pi, \pi^{\prime}, \psi\right), \quad \gamma\left(X, \pi, \pi^{\prime}, \psi\right),
$$

the local constants defined in [12].

The motivation for our next results is that the correspondence $\mathrm{V}$ does not preserve local constants of pairs of generic representations, for example it is not true that

$$
L\left(X, \pi, \pi^{\prime}\right)=L\left(X, \mathrm{~V}(\pi) \otimes_{\mathrm{ss}} \mathrm{V}\left(\pi^{\prime}\right)\right),
$$

see Example 6.12. In Definition 6.7, we define an injective map:

$$
\mathrm{CV}:\{\mathrm{V} \text {-parameters }\} \longrightarrow\left\{\begin{array}{c}
\text { Equivalence classes of } \mathrm{W}_{F^{-} \text {-semisimple }} \\
\text { Deligne } \overline{\mathbb{F}}_{\ell} \text {-representations of } \mathrm{W}_{F}
\end{array}\right\}
$$

which is not the natural inclusion, we call an element in its image a C-parameter. Explicitly, a $\mathrm{V}$-parameter $(\Phi, U)$ can be written as:

$$
(\Phi, U)=\bigoplus_{i \geqslant 1} \bigoplus_{k=0}^{o(\Psi)-1} a_{i, k}[0, i-1] \otimes \nu^{k} \Psi
$$

with all $a_{i, k}$ except possibly a finite number equal to zero, and we define $\operatorname{CV}(\Phi, U)$ by, for each $i$, replacing every instance of a complete cycle $\bigoplus_{k=0}^{o(\Psi)-1}[0, i-1] \otimes \nu^{k} \Psi$ with $[0, i-1] \otimes \mathcal{C}\left(\mathbb{Z}_{\Psi}\right)$ :

$$
\mathrm{CV}(\Phi, U)=\bigoplus_{i \geqslant 1} \bigoplus_{k=0}^{o(\Psi)-1} c_{i, k}[0, i-1] \otimes \nu^{k} \Psi \oplus \bigoplus_{i \geqslant 1} b_{i}[0, i-1] \otimes \mathcal{C}\left(\mathbb{Z}_{\Psi}\right)
$$

where $b_{i}=\min \left\{a_{i, k}: 0 \leqslant k \leqslant o(\Psi)-1\right\}$ and $c_{i, k}=a_{i, k}-b_{i}$ (see Section 6.3 for further details).

We can now state our main result, the first three properties of which are immediate consequence of their analogues for $\mathrm{V}$ and the definition of $\mathrm{CV}$ :

Main Theorem 2. [Lemma 6.11 and Theorem 6.13 For positive integers $n$, the bijections $\mathrm{C}=$ $\mathrm{CV} \circ \mathrm{V}$ :

$$
\left\{\begin{array}{c}
\text { Irreducible } \ell \text {-modular representations } \\
\text { of } \mathrm{GL}_{n}(F) \text { up to isomorphism }
\end{array}\right\} \stackrel{\mathrm{C}}{\longrightarrow}\{\text { C-parameters of dimension } n\}
$$

satisfy the following properties:

Let $\pi$ be an irreducible $\ell$-modular representation of $\mathrm{GL}_{n}(F)$.

(1) For all characters $\chi: \mathrm{GL}_{n}(F) \rightarrow{\overline{\mathbb{F}_{\ell}}}^{\times}$,

$$
\mathrm{C}(\chi \pi)=\chi \mathrm{C}(\pi)
$$

(2) Letting $c_{\pi}$ denote the central character of $\pi$, then via the Artin reciprocity map of local class field theory

$$
c_{\pi}=\operatorname{det}(\mathrm{C}(\pi)) .
$$


(3) Commutation with (smooth) duals:

$$
\mathrm{C}\left(\pi^{\vee}\right)=\mathrm{C}(\pi)^{\vee}
$$

(4) If $\pi$ is generic, for all positive integers $m$ and all generic $\ell$-modular representations $\pi^{\prime}$ of $\mathrm{GL}_{m}(F)$,

$$
\begin{aligned}
L\left(X, \pi, \pi^{\prime}\right) & =L\left(X, \mathrm{C}(\pi) \otimes_{\mathrm{ss}} \mathrm{C}\left(\pi^{\prime}\right)\right), \\
\gamma\left(X, \pi, \pi^{\prime}, \psi\right) & =\gamma\left(X, \mathrm{C}(\pi) \otimes_{\mathrm{ss}} \mathrm{C}\left(\pi^{\prime}\right), \psi\right), \\
\epsilon\left(X, \pi, \pi^{\prime}, \psi\right) & =\epsilon\left(X, \mathrm{C}(\pi) \otimes_{\mathrm{ss}} \mathrm{C}\left(\pi^{\prime}\right), \psi\right) .
\end{aligned}
$$

In Example 6.10, we give examples of C-parameters of special classes of representations.

As a corollary of our results, if one defines the $L, \epsilon, \gamma$-factors of a $\mathrm{V}$-parameter $\Phi$ to be the local constants of the corresponding C-parameter $\mathrm{CV}(\Phi)$, then we have a preservation of local factors for generic representations result for $\mathrm{V}$. However, as this construction goes via the associated C-parameter it feels more natural to us to state the result in terms of C.

It is tempting to say that $\mathrm{C}$ should preserve local factors of pairs of irreducible $\ell$-modular representations of $\mathrm{GL}_{n}(F)$ beyond the generic setting, for example the Godement-Jacquet local factors defined in [13. This holds for $n=2$ for the factors of [ibid.], thanks to the explicit computations of Mínguez in this case.

Vignéras' correspondence is characterized by a non-naive, but precise compatibility with reduction modulo $\ell$. At present there could be other correspondences to $\mathrm{C}$ which satisfy preservation of local factors, and even after fixing the set of C-parameters it is not clear whether or not the list of properties of Theorem 2 characterize the correspondence. We leave this question for future work.

Finally, we note other complementary results in the literature. Dat has partially realized Vignéras' correspondence (denoted V above) in cohomology in [5, 6]. Starting with Vignéras' correspondence in the supercuspidal case, Emerton and Helm [9] have introduced a modified mod $\ell$ local Langlands correspondence; this has a different flavour: it is a map from (isomorphism classes of) $n$-dimensional continuous representations of the absolute Galois group of $F$ to a class of modular representations of $\mathrm{GL}_{n}(F)$ called essentially $A I G$ (which are not necessarily irreducible). It would be interesting to further elucidate the connections between these two correspondences beyond the supercuspidal case, and to understand the structure of the modular essentially AIG representations which currently are only fully understood when $n=2$ [11].

We now describe the structure of the paper: Section 2 introduces the most general notation of the paper. In Section 3 we recall elements from the representation theory of $\mathrm{W}_{F}$, paying special attention to the tensor product. The first key section is Section 4, where we introduce the definition of a Deligne representation and prove Main Theorem 1. In Section 5, we extend the definitions of Artin-Deligne local constants to our setting of V and C-parameters. The second key section is the final section which recalls the $\mathrm{V}$-correspondence, defines the $\mathrm{C}$-correspondence, and proves Main Theorem 2.

Acknowledgements. We thank the referee for their corrections and suggestions which have improved the article. We thank David Helm and Alberto Mínguez for useful conversations. This work was started at the Imperial College London and continued at the Université de Poitiers, and the authors would like to thank them for their hospitality. We would like to thank the Heilbronn Institute for Mathematical Research and the Université de Poitiers for supporting these visits. We finally thank the anonymous referee for his helpful comments allowing to clarify some parts 
of the paper. The second author benefited from support from the grant ANR-13-BS01-0012 FERPLAY.

\section{General Notation}

Let $F$ be a non-archimedean local field with finite residue field $k_{F}$ of characteristic $p$ and cardinality $q$. For a positive integer $n$, we denote by $F_{n}$ the unique (up to isomorphism) unramified extension of $F$ of degree $n$.

Let $\ell$ be a prime number different from $p$. We fix an algebraic closure $\overline{\mathbb{Q}_{\ell}}$ of the $\ell$-adic numbers $\mathbb{Q}_{\ell}$. We denote by $\overline{\mathbb{Z}_{\ell}}$ the ring of integers of $\overline{\mathbb{Q}_{\ell}}$ and by $\mathfrak{m}$ its maximal ideal. We put $\overline{\mathbb{F}_{\ell}}=\overline{\mathbb{Z}_{\ell}} / \mathfrak{m}$, it is an algebraic closure of the finite field $\mathbb{F}_{\ell}$ with $\ell$ elements.

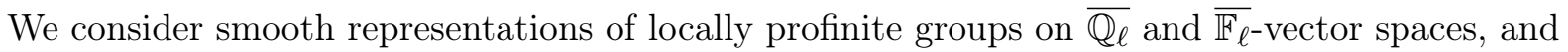
the connections between them. Henceforth, all representations considered are implicitly assumed to be smooth and, for convenience of stating results which apply to both cases $\overline{\mathbb{Q}_{\ell}}$ and $\overline{\mathbb{F}_{\ell}}$, we let $R$ denote either field. We abbreviate "representation on an $R$-vector space" to $R$-representation. When $R=\overline{\mathbb{F}_{\ell}}$ we say we are in the $\ell$-modular case, and when $R=\overline{\mathbb{Q}_{\ell}}$ we say we are in the $\ell$-adic case.

Let $H$ be a locally profinite group. Let $\operatorname{Rep}(H, R)$ denote the abelian category of $R$-representations of $H$, and $\operatorname{Irr}(H, R)$ denote the set of isomorphism classes of irreducible $R$-representations of $H$. Let $\pi, \pi^{\prime}$ be $R$-representations of $H$. We denote by $\pi^{\vee}$ the (smooth) contragredient of $\pi$, and put

$$
\operatorname{End}_{H}(\pi)=\operatorname{Hom}_{H}(\pi, \pi) \text {, and } \operatorname{Iso}_{H}\left(\pi, \pi^{\prime}\right)=\left\{\phi \in \operatorname{Hom}_{H}\left(\pi, \pi^{\prime}\right): \phi \text { is bijective }\right\} .
$$

Let $K$ be a closed subgroup of $H$. We fix a square root of $q$ in $\overline{\mathbb{Z}_{\ell}}$ and take its image in $\overline{\mathbb{F}_{\ell}}$ so we have a fixed square root of $q$ in $R$, which is compatible with reduction modulo $\ell$. We denote by

$$
\operatorname{Ind}_{K}^{H}: \operatorname{Rep}(K, R) \rightarrow \operatorname{Rep}(H, R)
$$

the usual normalized (with respect to this square root of $q$ ) induction functor.

Strictly speaking an $R$-representation consists of a pair $(\Phi, V)$ with $V$ an $R$-vector space and

$$
\Phi: H \rightarrow \mathrm{GL}_{R}(V)
$$

a group homomorphism. However, as we have done already, we will often denote the pair $(\Phi, V)$ just by $\Phi$, and in this case we will use $V_{\Phi}$, or even $\Phi$, to denote the underlying vector space upon which $H$ acts via $\Phi$. We also often make no distinction between an $R$-representation and its isomorphism class. Finally, we will write $\Phi \in \operatorname{Rep}(H, R)$ for $\Phi$ is an object in the category $\operatorname{Rep}(H, R)$, i.e. an $R$-representation of $H$.

\section{Representations of $\mathrm{W}_{F}$}

\subsection{Notation}

We refer to [3, Chapter 7] for the definitions and facts stated here concerning Weil groups. We fix a separable algebraic closure $\bar{F}$ of $F$, and will suppose that all finite extensions we consider 
are contained in $\bar{F}$. For a finite extension $E / F$ we let $\mathrm{G}_{E}=\operatorname{Gal}(\bar{F} / E)$ denote the absolute Galois group of $E ; I_{E}$ denote the inertia subgroup of $\mathrm{G}_{E} ; P_{E}$ denote the wild inertia subgroup, it is the pro- $p$ Sylow subgroup of $I_{E} ; \mathrm{W}_{E}$ denote the Weil group of $E$. We fix a geometric Frobenius element Frob in $\mathrm{W}_{F}$. We have

$$
\mathrm{W}_{F}=I_{F} \rtimes \mathrm{Frob}^{\mathbb{Z}},
$$

and, in particular, $\mathrm{W}_{F}$ is unimodular as $I_{F}$ is compact.

If $E$ is a finite extension of $F$, and $\pi$ is a representation of $\mathrm{W}_{F}$, we denote by $\pi_{E}$ the restriction of $\pi$ to $\mathrm{W}_{E}$. An $R$-representation of $\mathrm{W}_{F}$ is called unramified if it is trivial on $I_{F}$. If such a representation is irreducible, then it is necessarily a character as $\mathrm{W}_{F} / I_{F}$ is abelian. In fact, if $\Psi$ is an irreducible representation of $\mathrm{W}_{F}$ such that $\Psi^{I_{F}}$ (the $I_{F}$-fixed subspace of $\Psi$ ) is nonzero, then $\Psi^{I_{F}}$ is a nonzero $\mathrm{W}_{F}$-invariant subspace of $\Psi$, hence $\Psi=\Psi^{I_{F}}$ is an unramified character. We denote by $\nu$ the unramified character of $\mathrm{W}_{F}$ which satisfies $\nu($ Frob $)=q^{-1}$, and by $X^{u}\left(\mathrm{~W}_{F}\right)$ the group of unramified characters of $\mathrm{W}_{F}$.

An $R$-irreducible representation of $\mathrm{W}_{F}$ is called tamely ramified if it is trivial on $P_{F}$. We denote by $\operatorname{Irr}{ }^{\operatorname{tr}}\left(\mathrm{W}_{F}, R\right)$ the set of isomorphism classes of tamely ramified irreducible representations of $\mathrm{W}_{F}$.

Let $\Psi \in \operatorname{Irr}\left(\mathrm{W}_{F}, R\right)$, we put

$$
\mathbb{Z}_{\Psi}=\left\{\nu^{k} \Psi / \simeq, k \in \mathbb{Z}\right\}
$$

and call such a set of isomorphism classes an irreducible line. When $R=\overline{\mathbb{F}_{\ell}}$, the set $\mathbb{Z}_{\Psi}$ is finite, and we denote by $o(\Psi)$ its cardinality

$$
o(\Psi)=\left|\mathbb{Z}_{\Psi}\right|
$$

The integer $o(\Psi)$ clearly divides the order of $\nu$ which is the order of $q$ modulo $\ell$. In particular $o(\Psi)$ divides $\ell-1$ and is hence prime to $\ell$. We say that $\Psi$ is banal if $o(\Psi)>1$, that is $\Psi \neq \Psi \nu$.

\subsection{Irreducible representations of $\mathrm{W}_{F}$}

We now recall a description of the irreducible $R$-representations of $\mathrm{W}_{F}$ as induced representation which is well suited to studying congruences between $\overline{\mathbb{Q}_{\ell}}$-representations.

Let $E$ be a finite Galois extension of $F$ and $\pi \in \operatorname{Irr}\left(\mathrm{W}_{E}, R\right)$. For $\sigma \in \operatorname{Gal}(E / F) \simeq \mathrm{W}_{F} / \mathrm{W}_{E}$, we denote by $\pi^{\sigma}$ the $R$-representation of $\mathrm{W}_{E}$ defined on the same underlying space as $\pi$ by

$$
\pi^{\sigma}(w)=\pi\left(\sigma w \sigma^{-1}\right),
$$

for all $w \in \mathrm{W}_{E}$.

We say that $\pi$ is $\operatorname{Gal}(E / F)$-regular if the set

$$
\left\{\pi^{\sigma}: \sigma \in \operatorname{Gal}(E / F)\right\} \subseteq \operatorname{Irr}\left(\mathrm{W}_{E}, R\right)
$$

is of cardinality $|\operatorname{Gal}(E / F)|$.

Lemma 3.1. Let $E$ be a finite Galois extension of $F$ and $\tau$ an $R$-representation of $\mathrm{W}_{F}$. Then $\operatorname{Ind}_{\mathrm{W}_{E}}^{\mathrm{W}_{F}}(\tau)$ is irreducible if and only if $\tau$ is irreducible and $\operatorname{Gal}(E / F)$-regular. 
Proof. If $\operatorname{Ind}_{\mathrm{W}_{E}}^{\mathrm{W}_{F}}(\tau)$ is irreducible then $\tau$ is as well, by exactness of the induction functor. Moreover its endomorphism algebra is isomorphic to $R$ by Schur's lemma. However by Frobenius reciprocity and Mackey theory we have:

$$
\begin{aligned}
& \operatorname{End}_{W_{F}}\left(\operatorname{Ind}_{\mathrm{W}_{E}}^{\mathrm{W}_{F}}(\tau)\right) \simeq \operatorname{Hom}_{W_{E}}\left(\left.\operatorname{Ind}_{\mathrm{W}_{E}}^{\mathrm{W}_{F}}(\tau)\right|_{W_{E}}, \tau\right) \\
& \simeq \operatorname{Hom}_{W_{E}}\left(\bigoplus_{\sigma \in \operatorname{Gal}(E / F)} \tau^{\sigma}, \tau\right) \\
& \simeq \prod_{\sigma \in \operatorname{Gal}(E / F)} \operatorname{Hom}_{W_{E}}\left(\tau^{\sigma}, \tau\right) \text {. }
\end{aligned}
$$

This latter ring is isomorphic to $R$ if and only if $\tau$ is regular.

Conversely, if $\tau$ is irreducible and $\operatorname{Gal}(E / F)$-regular, let $W$ be a nonzero $\mathrm{W}_{F}$-stable subspace of $\operatorname{Ind}_{\mathrm{W}_{E}}^{\mathrm{W}_{F}}(\tau)$. Then it is a $\mathrm{W}_{E}$-stable subspace of $\bigoplus_{\sigma \in \operatorname{Gal}(E / F)} \tau^{\sigma}$ by Mackey theory. In particular as the regularity assumption implies that this direct sum is the decomposition into isotypic components of $\left.\operatorname{Ind}_{\mathrm{W}_{E}}^{\mathrm{W}_{F}}(\tau)\right|_{\mathrm{W}_{E}}$, the space $W$ contains $\tau^{\sigma}$ for some $\sigma$. As it is stable under $\mathrm{W}_{F}$, it contains all $\tau^{\sigma}$, hence it is equal to $\operatorname{Ind}_{\mathrm{W}_{E}}^{\mathrm{W}_{F}}(\tau)$.

We recall the following well known description of the irreducible $R$-representations of $\mathrm{W}_{F}$. It is a consequence of [4, Lemma 2.3, Proposition 2.1] in the $\ell$-adic case, and of [25, 2.6] in the $\ell$-modular case.

Theorem 3.2. (1) Let $\Psi \in \operatorname{Irr}^{\operatorname{tr}}\left(\mathrm{W}_{F}, R\right)$ of dimension $n$, then there is a $\operatorname{Gal}\left(F_{n} / F\right)$-regular tamely ramified character $\chi$ of $\mathrm{W}_{F_{n}}$ such that

$$
\Psi=\operatorname{Ind}_{\mathrm{W}_{F_{n}}}^{\mathrm{W}_{F}}(\chi)
$$

the character $\chi$ is unique up to conjugation by $\operatorname{Gal}\left(F_{n} / F\right)$.

(2) Let $\Psi \in \operatorname{Irr}\left(\mathrm{W}_{F}, R\right)$, then there exist a (finite) tamely ramified extension $E$ of $F, \Psi^{\mathrm{tr}} \in$ $\operatorname{Irr}^{\operatorname{tr}}\left(\mathrm{W}_{E}, R\right)$, and $\tau \in \operatorname{Irr}\left(\mathrm{W}_{E}, R\right)$ which restricts irreducibly to $P_{F}$, such that

$$
\Psi=\operatorname{Ind}_{\mathrm{W}_{E}}^{\mathrm{W}_{F}}\left(\Psi^{\mathrm{tr}} \otimes \tau\right),
$$

the representation $\Psi^{\operatorname{tr}} \otimes \tau$ is unique up to to conjugation by $\operatorname{Gal}(E / F)$. Moreover for fixed $\Psi$ and $\tau$, the representation $\Psi^{\text {tr }}$ is unique.

For $\Psi \in \operatorname{Irr}\left(\mathrm{W}_{F}, R\right)$, we denote by $R(\Psi)$ the group of unramified characters of $\mathrm{W}_{F}$ fixing $\Psi$ :

$$
R(\Psi)=\left\{\mu \in X^{u}\left(\mathrm{~W}_{F}\right), \mu \Psi=\Psi\right\} .
$$

Corollary 3.3. Let $\Psi \in \operatorname{Irr}\left(\mathrm{W}_{F}, R\right)$ and write $\Psi=\operatorname{Ind}_{\mathrm{W}_{E}}^{\mathrm{W}_{F}}\left(\Psi^{\operatorname{tr}} \otimes \tau\right)$ and $\Psi^{t r}=\operatorname{Ind}_{\mathrm{W}_{E_{n}}}^{\mathrm{W}_{E}}(\chi)$, for $E / F$ tamely ramified, a positive integer $n$, and $\chi$ a $\operatorname{Gal}\left(E_{n} / E\right)$-regular tamely ramified character of $\mathrm{W}_{E_{n}}$ as in Theorem 3.2. Put $r=\left[E_{n}: F\right]$, and $e=e\left(E_{n} / F\right)$ the ramification index of $E_{n} / F$. Then the map $\mu \mapsto \mu($ Frob $)$ is an isomorphism between $R(\pi)$ and the group of $r / e$-th roots of unity in $R^{\times}$.

Proof. The integer $e$ is also the ramification index of $E / F$ and we set $t=[E: F]$. One can ${\text { take } \text { Frob }_{E}=\text { Frob }^{t / e} \text { (where Frob }}_{E}$ stands for a geometric Frobenius element in $\mathrm{W}_{E}$ ). Then an unramified character $\mu: W_{F} \rightarrow R^{\times}$belongs to $R(\Psi)$ if and only if

$$
\operatorname{Ind}_{\mathrm{W}_{E}}^{\mathrm{W}_{F}}\left(\mu_{E} \Psi^{\mathrm{tr}} \otimes \tau\right)=\operatorname{Ind}_{\mathrm{W}_{E}}^{\mathrm{W}_{F}}\left(\Psi^{\mathrm{tr}} \otimes \tau\right),
$$


which is equivalent to

$$
\mu_{E} \Psi^{\mathrm{tr}}=\Psi^{\mathrm{tr}} .
$$

This is in turn equivalent to $\mu_{E_{n}} \chi$ is conjugate to $\chi$ by $\operatorname{Gal}\left(E_{n} / E\right)$, i.e. $\mu_{E_{n}}$ is of the form $\chi^{\sigma} / \chi$ for $\sigma \in \operatorname{Gal}\left(E_{n} / E\right)$. Now $\operatorname{Frob}_{E_{n}}^{\sigma} / \operatorname{Frob}_{E_{n}} \in I_{E_{n}}$, hence $\mu_{E_{n}}$ is trivial on Frob $E_{n}$, hence trivial as it is unramified. As one can take Frob $_{E_{n}}=\left(\operatorname{Frob}_{E}\right)^{n}=\operatorname{Frob}^{n t / e}$ :

$$
\mu \in R(\Psi) \Leftrightarrow \mu_{E_{n}}=1 \Leftrightarrow \mu_{E_{n}}\left(\operatorname{Frob}_{E_{n}}\right)=1 \Leftrightarrow \mu(\text { Frob })^{n t / e}=1 \Leftrightarrow \mu(\text { Frob })^{r / e}=1 .
$$

As an immediate consequence we have:

Corollary 3.4. With the same notation as Corollary 3.3, we obtain that $o(\Psi)=o\left(\nu^{r / e}\right)=o\left(\nu_{E}^{n}\right)$.

\subsection{Lattices and reduction modulo $\ell$}

An $\ell$-adic finite dimensional representation $\Theta$ of $\mathrm{W}_{F}$ is called integral if there is a $\overline{\mathbb{Z}_{\ell}}\left[\mathrm{W}_{F}\right]$-stable lattice in $V$. In general, when we say a lattice in the space of an integral representation of $\mathrm{W}_{F}$, we are always referring to a $\mathrm{W}_{F}$-stable lattice. We denote by $\operatorname{Irr}\left(\mathrm{W}_{F}, \overline{\mathbb{Q} \ell}\right)_{e}$ the set of isomorphism classes of integral irreducible representations of $\mathrm{W}_{F}$.

By [3, Proposition 28.6], if $\Theta \in \operatorname{Irr}\left(\mathrm{W}_{F}, \overline{\mathbb{Q}_{\ell}}\right)$, then there is an unramified character $\mu$ of $\mathrm{W}_{F}$ such that $\mu \Theta$ extends to an element of $\operatorname{Irr}\left(\mathrm{G}_{F}, \overline{\mathbb{Q}}\right)$. As the group $\mathrm{G}_{F}$ is profinite, the representation $\mu \Theta$ is automatically integral, and we deduce that $\Theta$ is integral if and only if $\mu$ takes values in $\overline{\mathbb{Z}}_{\ell} \times$. As explained in [20, 1.8], this happens if and only if $\operatorname{det}(\Theta)$ takes values in $\overline{\mathbb{Z}}_{\ell} \times$, hence one deduces the well known fact:

Lemma 3.5 ([20, Lemma 1.9]). The representation $\Theta \in \operatorname{Irr}\left(\mathrm{W}_{F}, \overline{\mathbb{Q}_{\ell}}\right)$ is integral if and only if $\operatorname{det}(\Theta)$ is integral.

Let $\Theta \in \operatorname{Irr}\left(\mathrm{W}_{F}, \overline{\mathbb{Q}_{\ell}}\right)_{e}$ and choose a lattice $L$ in $V_{\Theta}$. In this setting, we have a Brauer-Nesbitt principle:

Lemma 3.6 ([20,1.10]). The semisimplification of the $\overline{\mathbb{F}_{\ell}}$-representation $L / \mathfrak{m} L$ is independent of the choice of $L$.

(In fact, we did not need to assume $\Theta$ was irreducible, finite length would have been enough.) We let $r_{\ell}(\Theta)=(L / \mathfrak{m} L)_{\text {ss }}$ denote this semisimple $\overline{\mathbb{F}_{\ell}}$-representation. If $r_{\ell}(\Theta)$ is irreducible, we let

$$
\bar{\Theta}=r_{\ell}(\Theta)
$$

and say that $\Theta$ lifts $\bar{\Theta}$.

Suppose now $\Psi \in \operatorname{Irr}\left(\mathrm{W}_{F}, \overline{\mathbb{F}_{\ell}}\right)$ (because we treat $\ell$-adic and $\ell$-modular representations uniformly, in both cases we will often use $\Psi$ for an element of $\operatorname{Irr}\left(\mathrm{W}_{F}, \overline{\mathbb{F}_{\ell}}\right)$, but when needed for reduction modulo $\ell$, we will use $\Theta$ in the $\ell$-adic case as we did above). In this case, one can always lift $\Psi$ to an irreducible representation in $\operatorname{Irr}\left(\mathrm{W}_{F}, \overline{\mathbb{Q}_{\ell}}\right)_{e}$, this follows from the description of $\operatorname{Irr}\left(\mathrm{W}_{F}, R\right)$ given in Theorem 3.2, and is explained in [25, 2.6]. While not unique in general, when we wish to choose a lift of $\Psi$ to $\operatorname{Irr}\left(\mathrm{W}_{F}, \overline{\mathbb{Q}_{\ell}}\right)_{e}$ we will denote it by $\tilde{\Psi}$.

On the other hand, it is not true that the reduction modulo $\ell$ of a representation $\Theta \in \operatorname{Irr}\left(\mathrm{W}_{F}, \overline{\mathbb{Q}_{\ell}}\right)_{e}$ is irreducible in general. However it is well understood, and described in [20, 1.16]. We first fix 
some notation. Take $\Theta \in \operatorname{Irr}\left(\mathrm{W}_{F}, \overline{\mathbb{Q}_{\ell}}\right)_{e}$ and let $\beta$ be an integral unramified character of $\mathrm{W}_{F}$ such that $\beta \Theta$ extends to $\mathrm{G}_{F}$. Write $\Theta=\operatorname{Ind}_{\mathrm{W}_{E}}^{\mathrm{W}_{F}}\left(\Theta^{\text {tr }} \otimes \tau\right)$ with $\Theta^{\text {tr }}=\operatorname{Ind}_{\mathrm{W}_{E_{n}}}^{\mathrm{W}_{E}}(\chi)$ for some positive $n$ and some $\operatorname{Gal}\left(E_{n} / E\right)$-regular tamely ramified character $\chi$ of $\mathrm{W}_{E_{n}}$, as in Theorem 3.2. Both $\Theta^{\text {tr }}$ and $\chi$ are integral, and $\chi^{\prime}=\beta_{E_{n}} \chi$ is a character of finite order, which can uniquely be written $\chi^{\prime}=\alpha_{E_{n}} \chi_{\ell}^{\prime}$ for $\alpha$ a $\operatorname{Gal}\left(E_{d} / E\right)$-regular character of $\mathrm{W}_{E_{d}}$ where $d$ is a divisor of $n$, and $\chi_{\ell}^{\prime}$ is of order a power of $\ell$. The representation $\operatorname{Ind}_{\mathrm{W}_{E_{d}}}^{\mathrm{W}_{E}}(\bar{\alpha})$ is an irreducible representation of $\mathrm{W}_{E}$, so

$$
\Psi(\alpha, \beta)={\overline{\beta_{E}}}^{-1} \operatorname{Ind}_{\mathrm{W}_{E_{d}}}^{\mathrm{W}_{E}}(\bar{\alpha})=\operatorname{Ind}_{\mathrm{W}_{E_{d}}}^{\mathrm{W}_{E}}\left(\overline{\beta_{E_{d}}^{-1} \alpha}\right)
$$

is too. The representation $r_{\ell}(\tau)$ is also irreducible, and we thus write it $\bar{\tau}$. The quotient $m=n / d$ is either equal to 1 , or of the form $o\left(\nu_{E}^{d}\right) \ell^{a}$ for an integer $a \geqslant 0$.

Proposition 3.7. Let $\Theta \in \operatorname{Irr}\left(\mathrm{W}_{F}, \overline{\mathbb{Q}_{\ell}}\right)_{e}$, then under the notation above one has

$$
r_{\ell}(\Theta)=\bigoplus_{k=0}^{m-1} \nu^{k} \operatorname{Ind}_{\mathrm{W}_{E}}^{\mathrm{W}_{F}}(\Psi(\alpha, \beta) \otimes \bar{\tau})
$$

where $\operatorname{Ind}_{\mathrm{W}_{E}}^{\mathrm{W}_{F}}(\Psi(\alpha, \beta) \otimes \bar{\tau})$ is irreducible.

According to Corollary 3.4, we can restate Proposition 3.7 in the following less precise but simpler form:

Proposition 3.8. Let $\Theta \in \operatorname{Irr}\left(\mathrm{W}_{F}, \overline{\mathbb{Q}_{\ell}}\right)_{e}$, then either $r_{\ell}(\Theta)$ is irreducible, or it is of the form

$$
r_{\ell}(\Theta)=\ell^{a}\left(\bigoplus_{k=0}^{o(\Psi)-1} \nu^{k} \Psi\right)
$$

for $a \geqslant 0$ and $\Psi \in \operatorname{Irr}\left(\mathrm{W}_{F}, \overline{\mathbb{F}_{\ell}}\right)$.

Proof. In Proposition 3.7, the integer $m$ is of the form $o\left(\nu_{E}^{d}\right) \ell^{a}$ when not equal to 1 , but $\Psi=\operatorname{Ind}_{\mathrm{W}_{E}}^{\mathrm{W}_{F}}(\Psi(\alpha, \beta) \otimes \bar{\tau})$ satisfies $o(\Psi)=o\left(\nu_{E}^{d}\right)$ thanks to Corollary 3.4. The result is now a consequence of the definition of $o(\Psi)$.

We will denote by $\mathrm{St}_{a}\left(\mathbb{Z}_{\Psi}\right)$ the representation $\ell^{a}\left(\bigoplus_{k=0}^{o(\Psi)-1} \nu^{k} \Psi\right)$ of the above proposition.

We deduce the following corollary we shall use later:

Corollary 3.9. Let $\Theta \in \operatorname{Irr}\left(\mathrm{W}_{F}, \overline{\mathbb{Q}_{\ell}}\right)_{e}$. Then the following are equivalent:

(1) $r_{\ell}(\Theta)^{I_{F}} \neq 0$.

(2) There is a positive integer $n$, equal to 1 or otherwise of the form $n=o(\nu) \ell^{r}$ with $r \geqslant 0$, and an integral character $\chi$ of $\mathrm{W}_{E_{n}}$ with $\bar{\chi}=\mu_{E_{n}}$ for $\mu$ an unramified character of $\mathrm{W}_{F}$, such that

$$
\Theta=\operatorname{Ind}_{\mathrm{W}_{F_{n}}}^{\mathrm{W}_{F}}(\chi) \text {. }
$$

When they are satisfied we have

$$
r_{\ell}(\Theta)^{I_{F}}=r_{\ell}(\Theta)=\bigoplus_{k=0}^{n-1} \nu^{k} \mu=\ell^{r}\left(\bigoplus_{k=0}^{o(\nu)-1} \nu^{k} \mu\right)
$$


Proof. If $\Theta=\operatorname{Ind}_{\mathrm{W}_{F_{n}}}^{\mathrm{W}_{F}}(\chi)$ with $\chi$ as in the statement, then

$$
r_{\ell}(\Theta)^{I_{F}}=r_{\ell}(\Theta)=\sum_{k=0}^{n-1} \nu^{k} \mu
$$

thanks to Proposition 3.7. Conversely, suppose that $r_{\ell}(\Theta)^{I_{F}} \neq 0$. Write $\Theta=\operatorname{Ind}_{\mathrm{W}_{E}}^{\mathrm{W}_{F}}\left(\Theta^{\operatorname{tr}} \otimes \tau\right)$ and $\Theta^{\mathrm{tr}}=\operatorname{Ind}_{\mathrm{W}_{E_{n}}}^{\mathrm{W}_{E}}(\chi)$ as discussed before Proposition 3.7. From the same proposition, we have

$$
r_{\ell}(\Theta)=\bigoplus_{k=0}^{m-1} \nu^{k} \operatorname{Ind}_{\mathrm{W}_{E}}^{\mathrm{W}_{F}}(\Psi(\alpha, \beta) \otimes \bar{\tau})
$$

with all of the $\nu^{k} \operatorname{Ind}_{\mathrm{W}_{E}}^{\mathrm{W}_{F}}(\Psi(\alpha, \beta) \otimes \bar{\tau})$ irreducible. However one of them has nonzero $I_{F}$-fixed subspace by assumption, so they must all be unramified characters. The fact that they are characters implies that $F=E=E_{d}$, i.e. $d=1$, and that $\chi=\left(\beta^{-1} \alpha\right)_{E_{n}} \chi_{\ell}^{\prime}$, so that $\bar{\chi}=$ $\left(\overline{\beta^{-1} \alpha}\right)_{E_{n}}$. Moreover setting $\mu=\overline{\beta^{-1} \alpha}$, the character $\mu_{E_{n}}$ must be unramified, hence $\mu$ as well, as $I_{E_{n}}=I_{F}$. This proves the converse implication.

\subsection{Tensor products of $\ell$-modular representations of $\mathrm{W}_{F}$}

We now study $\Psi \otimes \Psi^{\prime}$ when $\Psi$ and $\Psi^{\prime}$ belong to $\operatorname{Irr}\left(\mathrm{W}_{F}, \overline{\mathbb{F}_{\ell}}\right)$. First we observe some fundamental differences with the case of characteristic zero, where such a tensor product is semisimple: this will have consequences later in the paper, we will have to define a semisimple tensor product for $\mathrm{W}_{F}$-semisimple Deligne representations. In fact when $R=\overline{\mathbb{Q}_{\ell}}$, by [3, Proposition 28.7], a finite dimensional representation $\Phi$ of $\mathrm{W}_{F}$ is semisimple if and only if it is Frobenius semisimple, i.e. if and only if $\Phi$ (Frob) is semisimple. As an immediate consequence, the tensor product of two semisimple $\overline{\mathbb{Q}_{\ell}}$-representations of $\mathrm{W}_{F}$ is itself semisimple. In the $\ell$-modular case the situation is totally different: in general, Frobenius semisimple does not imply semisimple, neither does semisimple imply Frobenius semisimple, and the tensor product does not preserve semisimplicity! We give a list of examples illustrating these differences, we use the following lemma:

Lemma 3.10. Let $E / F$ be a quadratic extension and $\ell=2$. The $\overline{\mathbb{F}_{\ell}}$-representation $\operatorname{Ind}_{\mathrm{W}_{E}} \mathrm{~W}_{W_{E}}\left(\mathbf{1}_{W_{E}}\right)$ is uniserial of length 2 , with both subquotients isomorphic to $\mathbf{1}_{\mathrm{W}_{F}}$.

Proof. It is two-dimensional, and contains $\mathbf{1}_{\mathrm{W}_{F}}$ as a submodule with multiplicity one by Frobenius reciprocity law. The quotient $\operatorname{Ind}_{\mathrm{W}_{E}}^{\mathrm{W}_{F}}\left(\mathbf{1}_{\mathrm{W}_{E}}\right) / \mathbf{1}_{\mathrm{W}_{F}}$ is a character $\mu$, so $\mu^{-1}$ is a submodule of $\operatorname{Ind}_{\mathrm{W}_{E}}^{\mathrm{W}_{F}}(\mathbf{1})$ which is self-dual. Hence by Frobenius reciprocity again $\mu_{E}=\mathbf{1}$ so $\mu^{2}=\mathbf{1}$, but the condition $\ell=2$ implies that $\mu$ is then trivial. All in all, $\operatorname{Ind}_{\mathrm{W}_{E}}^{\mathrm{W}_{F}}\left(\mathbf{1}_{\mathrm{W}_{E}}\right)$ is indecomposable with socle and cosocle $\mathbf{1}_{\mathrm{W}_{F}}$.

Of course, one can concoct similar examples for any prime $\ell$ using degree $\ell$ extensions.

Example 3.11. For these examples we take $\ell=2$. In all our examples we choose a separable quadratic extension $E / F$ with Galois involution $\sigma$; a character $\chi: \mathrm{W}_{E} \rightarrow{\overline{\mathbb{F}_{2}}}^{\times}$, and consider the 2-dimensional $\overline{\mathbb{F}_{2}}$-representation induced from $\chi$

$$
\Phi=\operatorname{Ind}_{\mathrm{W}_{E}}^{\mathrm{W}_{F}}(\chi) .
$$

We fix a geometric Frobenius element Frob ${ }_{E}$ in $\mathrm{W}_{E}$. 
By the Artin reciprocity isomorphism of local class field theory, we can identify the topologically abelianized quotient $\mathrm{W}_{E}^{\mathrm{ab}}$ of $\mathrm{W}_{E}$ with the group $E^{\times}$, and similarly $\mathrm{W}_{F}^{a b}$ with $F^{\times}$. Via this isomorphism the Frobenius elements Frob, Frob ${ }_{E}$ corresponds to uniformizers $\varpi, \varpi_{E}$ of $F$ and $E$ respectively. We also recall that the restriction functor from $\mathrm{W}_{F}^{\mathrm{ab}}$ to $\mathrm{W}_{E}^{\mathrm{ab}}$ (relative to the natural inclusion $\mathrm{W}_{E}^{a b}$ into $\mathrm{W}_{F}^{a b}$ ) corresponds to the norm $N_{E / F}: E^{\times} \rightarrow F^{\times}$. In the second example, we use this identification to go from characters of $\mathrm{W}_{F}$ and $\mathrm{W}_{E}$ to characters of $F^{\times}$and $E^{\times}$ respectively.

(1) Frobenius semisimple does not imply semisimple: We take $E / F$ ramified and $\chi=\mathbf{1}_{\mathrm{W}_{E}}$. Then Frob ${ }_{E}=$ Frob acts trivially on $\Phi$ as $\Phi_{E}=\mathbf{1}_{\mathrm{W}_{E}} \oplus \mathbf{1}_{\mathrm{W}_{E}}$, however $\Phi$ is not semisimple according to Lemma 3.10 .

(2) Semisimple does not imply Frobenius semisimple: take $F=\mathbb{Q}_{2}$ and $E=F_{2}$ the unique (up to isomorphism) quadratic unramified extension. Write

$$
E^{\times} \simeq \mathbb{Z} \times(\mathbb{Z} / 3 \mathbb{Z}) \times\left(1+2 \mathbb{Z}_{2}\right),
$$

and, by abuse of notation, we identify $E^{\times}$with this product. Notice that the norm $N_{k_{E} / k_{F}}$ is trivial as a character of $k_{E}^{\times}$because $k_{F}^{\times}=\{1\}$, in particular

$$
N_{E / F}(\mathbb{Z} / 3 \mathbb{Z})=\{1\} .
$$

We take $\chi$ a non trivial character of $\mathbb{Z} / 3 \mathbb{Z}$ with values in $\overline{\mathbb{F}_{2}}$ and extend it trivially on $\mathbb{Z}$ and extend it on $\left(1+2 \mathbb{Z}_{2}\right)$ to a character $\chi$ of $E^{\times}$. Then $\chi$ does not factor through $N_{E / F}$, but it satisfies $\chi\left(\varpi_{E}\right)=1$. The corresponding character $\chi$ of $\mathrm{W}_{E}$ is thus $\operatorname{Gal}(E / F)$-regular, but satisfies $\chi\left(\right.$ Frob $\left._{E}\right)=1$. Hence the representation $\operatorname{Ind}_{\mathrm{W}_{E}}^{\mathrm{W}_{F}}(\chi)$ is an irreducible representation of $\mathrm{W}_{F}$. If Frob acted via a scalar on this representation, then $\operatorname{Ind}_{\mathrm{W}_{E}}^{\mathrm{W}_{F}}(\chi)$ would be an irreducible representation of $I_{F}=I_{E}(E / F$ is unramified). This is absurd as its restriction to $\mathrm{W}_{E}$ is a direct sum of two lines. However Frob $_{E}$ acts trivially on $\operatorname{Ind}_{\mathrm{W}_{E}}^{\mathrm{W}_{F}}(\chi)_{E}=\chi \oplus \chi^{\sigma}$, and moreover one can take Frob ${ }_{E}=$ Frob $^{2}$.

We conclude that $\Phi$ is irreducible hence semisimple, $\Phi$ (Frob) is not a scalar hence not Id, but $\Phi(\text { Frob })^{2}=\mathrm{Id}$, hence $\Phi$ (Frob) is not semisimple because $\ell=2$.

$(3) \otimes$ does not preserve semi-simplicity: take $\chi$ to be $\operatorname{Gal}(E / F)$-regular, hence $\Phi$ and $\Phi^{\vee}$ are irreducible. The following decomposition of their tensor product, which follows from Mackey theory,

$$
\Phi \otimes \Phi^{\vee}=\operatorname{Ind}_{\mathrm{W}_{E}}^{\mathrm{W}_{F}}\left(\mathbf{1}_{\mathrm{W}_{E}}\right) \oplus \operatorname{Ind}_{\mathrm{W}_{E}}^{\mathrm{W}_{F}}\left(\chi^{-1} \chi^{\sigma}\right)
$$

and Lemma 3.10 show that it is not semisimple.

Remark 3.12. By the main result of [17], the tensor product of two semisimple $\overline{\mathbb{F}_{\ell}}$-representations of respective dimensions $d$ and $d^{\prime}$ is semisimple if $d+d^{\prime}-2<\ell$. In Example 3.11 (3) $d=d^{\prime}=\ell=2$ which just falls out of the range of Serre's result, so fortunately does not provide a counterexample!

If $\Phi$ and $\Phi^{\prime}$ are two semisimple representations of $\mathrm{W}_{F}$, we denote by

$$
\Phi \otimes_{\mathrm{ss}} \Phi^{\prime}=\left(\Phi \otimes \Phi^{\prime}\right)_{\mathrm{ss}}
$$

the semisimplifaction of their tensor product.

To end this section, we describe $\left(\Psi \otimes \Psi^{\prime}\right)^{I_{F}}$ when $\Psi$ and $\Psi^{\prime}$ are two irreducible banal $\overline{\mathbb{F}_{\ell^{-}}}$ representations of $\mathrm{W}_{F}$. It is the central result of this section. 
Proposition 3.13. Let $\Psi$ and $\Psi^{\prime}$ be two banal irreducible $\overline{\mathbb{F}_{\ell}}$-representations of $\mathrm{W}_{F}$, and let $\tilde{\Psi}$ and $\tilde{\Psi}^{\prime}$ be $\ell$-adic lifts of $\Psi$ and $\Psi^{\prime}$ respectively. Choose lattices $L$ in $V_{\tilde{\Psi}}$ and $L^{\prime}$ in $V_{\tilde{\Psi}^{\prime}}$, and put $M=L \otimes_{\overline{\mathbb{Z}_{\ell}}} L^{\prime}$. For any $\overline{\mathbb{Q}_{\ell}}$-subspace $W$ of $V_{\tilde{\Psi}} \otimes V_{\tilde{\Psi}^{\prime}}$, set $\bar{W}=(W \cap M) \otimes_{\overline{\mathbb{Z}_{\ell}}} \overline{\mathbb{F}_{\ell}}$. Then

$$
\overline{\left(V_{\tilde{\Psi}} \otimes V_{\tilde{\Psi}^{\prime}}\right)^{I_{F}}} \simeq\left(V_{\Psi} \otimes V_{\Psi^{\prime}}\right)^{I_{F}} \quad \text { and } \quad\left(\left(V_{\Psi} \otimes V_{\Psi^{\prime}}\right)^{I_{F}}\right)_{\mathrm{SS}}=\left(V_{\Psi} \otimes_{\mathrm{SS}} V_{\Psi^{\prime}}\right)^{I_{F}} .
$$

Proof. We write

$$
\tilde{\Psi} \otimes \tilde{\Psi}^{\prime}=\bigoplus_{i} \mu_{i} \oplus \bigoplus_{k} \Psi_{k}
$$

as a (finite) sum of unramified characters $\mu_{i}$ and ramified irreducible representations $\Psi_{k}$. In particular,

$$
\left(\tilde{\Psi} \otimes \tilde{\Psi}^{\prime}\right)^{I_{F}}=\bigoplus_{i} \mu_{i}
$$

The inclusion

$$
\overline{\left(V_{\tilde{\Psi}} \otimes V_{\tilde{\Psi}^{\prime}}\right)^{I_{F}}} \subseteq\left(V_{\Psi} \otimes V_{\Psi^{\prime}}\right)^{I_{F}}
$$

is clear. Suppose that the inclusion was strict, this would imply that one of the $\Psi_{k}$ 's is such that $\left(\overline{V_{\Psi_{k}}}\right)^{I_{F}}$ is nonzero. For the sake of contradiction we suppose that it is the case. According to Corollary 3.9, setting either $E=F$ or $E=F_{o(\nu) \ell^{m}}$ for $m \geqslant 0$, one has

$$
\Psi_{k}=\operatorname{Ind}_{\mathrm{W}_{E}}^{\mathrm{W}_{F}}(\chi)
$$

for $\chi$ an integral $\operatorname{Gal}(E / F)$-regular character of $E^{\times}$such that $r_{\ell}(\chi)=\mu_{E}$ for $\mu$ an unramified character of $\mathrm{W}_{F}$.

Now by definition, the space $\operatorname{Hom}_{\mathrm{W}_{F}}\left(\tilde{\Psi} \otimes \tilde{\Psi}^{\prime}, \operatorname{Ind}_{\mathrm{W}_{E}}^{\mathrm{W}_{F}}(\chi)\right)$ is nonzero. Take $U$ a nonzero element in this space, as $\operatorname{Ind}_{\mathrm{W}_{E}}^{\mathrm{W}_{F}}(\chi)$ is irreducible $U$ is surjective. By Mackey theory

$$
\left.\operatorname{Ind}_{\mathrm{W}_{E}}^{\mathrm{W}_{F}}(\chi)\right|_{\mathrm{W}_{E}}=\bigoplus_{s \in \mathrm{W}_{F} / \mathrm{W}_{E}} s \cdot D
$$

where $D$ is a line over $\overline{\mathbb{Q}_{\ell}}$ on which $\mathrm{W}_{E}$ acts as $\chi$, and $\mathrm{W}_{E}$ more generally acts on $s . D$ as $\chi^{s}$. Denote by $p$ the projection on $D$ with respect to $\bigoplus_{s \neq 1} s$. D, then $p(U(M))=O_{D}$ is a lattice in $D$ by [19, 9.3, (vi)], and

$$
N=\bigoplus_{s \in \mathrm{W}_{F} / \mathrm{W}_{E}} s \cdot O_{D}=\operatorname{Ind}_{\mathrm{W}_{E}}^{\mathrm{W}_{F}}\left(O_{D}\right)
$$

is a $\mathrm{W}_{F}$-stable lattice in $\operatorname{Ind}_{\mathrm{W}_{E}}^{\mathrm{W}_{F}}(\chi)$. Notice that for $s \in \mathrm{W}_{F} / \mathrm{W}_{E}$, the map $p_{s}=s \circ p \circ s^{-1}$ the projection on $D$ with respect to $\bigoplus_{s^{\prime} \neq s} s^{\prime}$. D. We have

$$
p_{s}(U(M))=s\left(p\left(U\left(s^{-1} \cdot M\right)\right)\right)=s(p(U(M)))=s . O_{D},
$$

hence

$$
U(M)=\bigoplus_{s \in \mathrm{W}_{F} / \mathrm{W}_{E}} p_{s}(U(M))=\bigoplus_{s \in \mathrm{W}_{F} / \mathrm{W}_{E}} s \cdot O_{D}=N .
$$

Tensoring by $\overline{\mathbb{F}_{\ell}}$, we obtain a surjective $\mathrm{W}_{F^{-}}$-intertwining operator $\bar{U}$ from $\Psi \otimes \Psi^{\prime}$ onto

$$
\operatorname{Ind}_{\mathrm{W}_{E}}^{\mathrm{W}_{F}}\left(r_{\ell}(\chi)\right)=\operatorname{Ind}_{\mathrm{W}_{E}}^{\mathrm{W}_{F}}\left(\mu_{E}\right)
$$

i.e. $\operatorname{Ind}_{\mathrm{W}_{E}}^{\mathrm{W}_{F}}\left(\mu_{E}\right)$ is a quotient of $\Psi \otimes \Psi^{\prime}$. Dualizing, we obtain that

$$
\operatorname{Ind}_{\mathrm{W}_{E}}^{\mathrm{W}_{F}}\left(\mu_{E}^{-1}\right)
$$


is a submodule of $\Psi^{\vee} \otimes \Psi^{\prime \vee}$. By Frobenius reciprocity, we also have for any $k \in \mathbb{Z}$ :

$$
\begin{aligned}
\operatorname{Hom}_{\mathrm{W}_{F}}\left(\mu^{-1} \nu^{-k}, \operatorname{Ind}_{\mathrm{W}_{E}}^{\mathrm{W}_{F}}\left(\mu_{E}^{-1}\right)\right) & \simeq \operatorname{Hom}_{\mathrm{W}_{E}}\left(\left(\mu^{-1} \nu^{-k}\right)_{E}, \mu_{E}^{-1}\right) \\
& \simeq \operatorname{Hom}_{E}\left(\mu_{E}^{-1}, \mu_{E}^{-1}\right) \simeq \overline{\mathbb{F}_{\ell}}
\end{aligned}
$$

Hence for any $k, \mu^{-1} \nu^{-k}$ is a submodule of $\operatorname{Ind}_{\mathrm{W}_{E}}^{\mathrm{W}_{F}}\left(\mu_{E}^{-1}\right)$ which is itself a submodule of $\Psi^{\vee} \otimes \Psi^{\prime \vee}$. Dualizing again, we deduce that $\mu \nu^{k}$ is a quotient of $\Psi \otimes \Psi^{\prime}$ for all $k$. However this would imply that $\Psi^{\prime \vee} \simeq \mu \nu^{k} \Psi$ for all $k$, and this would in particular imply that $\nu \Psi \simeq \Psi$ which is absurd by our banality assumption. Hence we just proved that

$$
\overline{\left(V_{\tilde{\Psi}} \otimes V_{\tilde{\Psi}^{\prime}}\right)^{I_{F}}} \simeq\left(V_{\Psi} \otimes V_{\Psi^{\prime}}\right)^{I_{F}} .
$$

In fact, we proved that $\bar{\Psi}_{k} I_{F}=\{0\}$ for all $\Psi_{k}$. However $r_{\ell}\left(\Psi_{k}\right)^{I_{F}}=\{0\}$ if and only if $\bar{\Psi}_{k} I_{F}=\{0\}$ according to Corollary 3.9 , hence the isomorphism

$$
\left[\left(V_{\Psi} \otimes V_{\Psi^{\prime}}\right)^{I_{F}}\right]_{\mathrm{Ss}} \simeq\left(V_{\Psi} \otimes_{\mathrm{Ss}} V_{\Psi^{\prime}}\right)^{I_{F}} .
$$

\section{Deligne representations}

In this section, we classify $\mathrm{W}_{F}$-semisimple Deligne representations (see Definition 4.1), in terms of irreducible representations of $\mathrm{W}_{F}$, up to a certain equivalence relation (Definition 4.6). The purpose of doing this is that in the $\ell$-modular case, we will parametrize (see Section 6.3 $\ell$ modular irreducible representations of $\mathrm{GL}_{n}(F)$ by equivalence classes of $\ell$-modular $n$-dimensional semisimple Deligne representations. The classification of $\mathrm{W}_{F}$-semisimple Deligne representations we obtain in the $\ell$-adic setting is well known, see [7, Proposition 3.1.3] and [16, Sections 3, 5].

\subsection{Definitions, notation and basic properties}

Here is our definition of Deligne representations, which as we shall see, specializes to the usual definition when $R=\overline{\mathbb{Q}_{\ell}}$.

Definition 4.1. A Deligne R-representation of $\mathrm{W}_{F}$ is a pair $(\Phi, U)$ where $\Phi$ is a finite dimensional $R$-representation of $\mathrm{W}_{F}$, and $U \in \operatorname{Hom}_{\mathrm{W}_{F}}(\nu \Phi, \Phi)$. Moreover, we say that $(\Phi, U)$ is a $\mathrm{W}_{F}$-semisimple Deligne $R$-representation if $\Phi$ is semisimple as an $R$-representation of $\mathrm{W}_{F}$.

Let $(\Phi, U),\left(\Phi^{\prime}, U^{\prime}\right)$ be Deligne $R$-representations of $\mathrm{W}_{F}$. We write

$$
\begin{aligned}
\operatorname{Hom}_{\mathrm{D}}\left((\Phi, U),\left(\Phi^{\prime}, U^{\prime}\right)\right) & =\left\{f \in \operatorname{Hom}_{F}\left(\Phi, \Phi^{\prime}\right): f \circ U=U^{\prime} \circ f\right\}, \\
\operatorname{End}_{\mathrm{D}}(\Phi, U) & =\operatorname{Hom}_{\mathrm{D}}((\Phi, U),(\Phi, U)), \\
\operatorname{Iso}_{\mathrm{D}}\left((\Phi, U),\left(\Phi^{\prime}, U^{\prime}\right)\right) & =\operatorname{Hom}_{\mathrm{D}}\left((\Phi, U),\left(\Phi^{\prime}, U^{\prime}\right)\right) \cap \operatorname{Isow}_{F}\left(\Phi, \Phi^{\prime}\right) .
\end{aligned}
$$

If this latter space is non-empty, we say that $(\Phi, U)$ and $\left(\Phi, U^{\prime}\right)$ are isomorphic. A Deligne subrepresentation of $(\Phi, U)$ is a subspace of $V_{\Phi}$ which is stable under $\Phi\left(\mathrm{W}_{F}\right)$ and $U$, considered as a Deligne $R$-representation.

We will sometimes write $V_{(\Phi, U)}$ for $V_{\Phi}$, and also $d_{(\Phi, U)}$ or $d_{\Phi}$ for $\operatorname{dim}_{R}\left(V_{\Phi}\right)$.

For $U$ an endomorphism of a finite dimensional $R$-vector space, we denote by $\operatorname{Spec}(U)$ the set of its eigenvalues. 
Remark 4.2. (1) Take $\Phi$ a finite dimensional representation of $\mathrm{W}_{F}$, and $U \in \operatorname{End}_{W_{F}}\left(V_{\Phi}\right)$ with Jordan decomposition $S+N$ ( $S$ semisimple and $N$ nilpotent). Then $U \in \operatorname{Hom}_{W_{F}}(\nu \Phi, \Phi)$ if and only if $S$ and $N$ are in $\operatorname{Hom}_{W_{F}}(\nu \Phi, \Phi)$.

(2) If $R=\overline{\mathbb{Q} \ell}$, and $U \in \operatorname{Hom}_{W_{F}}(\nu \Phi, \Phi)$, then $U=N$ is nilpotent as $\operatorname{Spec}(U)$ is stable under multiplication by $q$.

The Jordan decomposition of $U$ will always be denoted by $S+N$, unless explicitly stated.

As we only consider Deligne $R$-representations of $\mathrm{W}_{F}$, we will suppress the "of $\mathrm{W}_{F}$ " in our notation.

Definition 4.3. Let $(\Phi, U),\left(\Phi^{\prime}, U^{\prime}\right)$ be Deligne $R$-representations and let $U=S+N$.

(1) The direct sum of $(\Phi, U)$ and $\left(\Phi^{\prime}, U^{\prime}\right)$ is defined by

$$
(\Phi, U) \oplus\left(\Phi^{\prime}, U^{\prime}\right)=\left(\Phi \oplus \Phi^{\prime}, U \oplus U^{\prime}\right) .
$$

(2) The dual of $(\Phi, U)$ is defined by

$$
(\Phi, U)^{\vee}=\left(\Phi^{\vee}, S^{\vee}-N^{\vee}\right)
$$

(3) The tensor product of of $(\Phi, U)$ and $\left(\Phi^{\prime}, U^{\prime}\right)$ is defined by

$$
(\Phi, U) \otimes\left(\Phi^{\prime}, U^{\prime}\right)=\left(\Phi \otimes \Phi^{\prime}, U \otimes \operatorname{Id} \oplus \operatorname{Id} \otimes U^{\prime}\right) .
$$

Notation 4.4. We introduce the following notation:

- $\operatorname{Rep}_{\mathrm{ss}}(\mathrm{D}, R)$ for the set of isomorphism classes of $\mathrm{W}_{F}$-semisimple Deligne $R$-representations.

- $\operatorname{Indec}_{\mathrm{ss}}(\mathrm{D}, R)$ for the isomorphism classes of Deligne $R$-representations in $\operatorname{Rep}_{\mathrm{ss}}(\mathrm{D}, R)$ which are indecomposable under direct sum of Deligne $R$-representations.

- $\operatorname{Irr}_{\mathrm{ss}}(\mathrm{D}, R)$ for the isomorphism classes of irreducible Deligne $R$-representations in $\operatorname{Rep}_{\mathrm{ss}}(\mathrm{D}, R)$. (Note that $(\Phi, U) \in \operatorname{Irr}_{\mathrm{ss}}(\mathrm{D}, R)$ does not imply $\Phi \in \operatorname{Irr}\left(W_{F}, R\right)$.)

- $\operatorname{Nilp}_{\mathrm{ss}}(\mathrm{D}, R)$ for the isomorphism classes of Deligne $R$-representations $(\Phi, U) \in \operatorname{Rep}_{\mathrm{ss}}(\mathrm{D}, R)$ with $U=N$ nilpotent. In particular, by Remark $4.2(2), \operatorname{Rep}_{\mathrm{ss}}\left(\mathrm{D}, \overline{\mathbb{Q}_{\ell}}\right)=\operatorname{Nilp}_{\mathrm{ss}}\left(\mathrm{D}, \overline{\mathbb{Q}_{\ell}}\right)$.

Clearly, $\operatorname{Rep}_{\mathrm{ss}}(\mathrm{D}, R)$ is stable under direct sum and duals:

Lemma 4.5. For $(\Phi, U),\left(\Phi^{\prime}, U^{\prime}\right) \in \operatorname{Rep}_{\mathrm{ss}}(\mathrm{D}, R)$, then

$$
(\Phi, U) \oplus\left(\Phi^{\prime}, U^{\prime}\right),(\Phi, U)^{\vee} \in \operatorname{Rep}_{\mathrm{ss}}(\mathrm{D}, R) .
$$

In contrast, the set $\operatorname{Rep}_{\mathrm{ss}}(\mathrm{D}, R)$ is not stable under the tensor product of Deligne $R$-representations, according to Example 3.11. (3). To remedy this problem, we will introduce a semisimple tensor product $\otimes_{\mathrm{ss}}$ in Section 4.4. Of course, whenever the tensor product is $\mathrm{W}_{F}$-semisimple we will have $\otimes_{\mathrm{ss}}=\otimes$, which will be the case when the characteristic is not too small in relation to the dimensions of the representations by Remark 3.12 .

Now we introduce an equivalence relation $\sim$ on $\operatorname{Rep}_{\mathrm{ss}}(\mathrm{D}, R)$. We do this as we shall later parametrize irreducible representations of the group $\mathrm{GL}_{n}(F)$ by equivalence classes rather than isomorphism classes of Deligne $R$-representations. 
Definition 4.6. The definition is in two steps:

(1) Deligne $R$-representations $(\Phi, U),\left(\Phi^{\prime}, U^{\prime}\right) \in \operatorname{Indec}_{\mathrm{ss}}(\mathrm{D}, R)$ are equivalent, denoted

$$
(\Phi, U) \sim\left(\Phi^{\prime}, U^{\prime}\right)
$$

if there exists $\lambda \in R^{\times}$such that

$$
\left(\Phi^{\prime}, U^{\prime}\right)=(\Phi, \lambda U)
$$

(2) In the general case, $(\Phi, U),\left(\Phi^{\prime}, U^{\prime}\right) \in \operatorname{Rep}_{\mathrm{ss}}(\mathrm{D}, R)$ are equivalent, still denoted

$$
(\Phi, U) \sim\left(\Phi^{\prime}, U^{\prime}\right),
$$

if one can decompose $\left(\Phi^{\prime}, U^{\prime}\right)=\bigoplus_{i=1}^{r}\left(\Phi_{i}, U_{i}\right)$ and $(\Phi, U)=\bigoplus_{i=1}^{r}\left(\Phi_{i}, U_{i}\right)$ such that $\left(\Phi_{i}, U_{i}\right) \sim$ $\left(\Phi_{i}, U_{i}\right)$ in $\operatorname{Indec}_{\mathrm{ss}}(\mathrm{D}, R)$.

Remark 4.7. To see that Definition 4.6 defines an equivalence relation we use that the decomposition of a $\mathrm{W}_{F^{-}}$-semisimple Deligne $R$-representation into indecomposable (necessarily $\mathrm{W}_{F^{-}}$ semisimple) Deligne $R$-representations is unique. This will be a consequence of our classification of indecomposable $\mathrm{W}_{F}$-semisimple Deligne representations: we show as a consequence of Propositions 4.28 and 4.30 , and Theorems 4.29 that the endomorphism ring of such a representation is local, which is what is needed in the proof of the Krull-Schmidt theorem.

Notation 4.8. We introduce square brackets to denote equivalence classes:

- For $(\Phi, U) \in \operatorname{Rep}_{\mathrm{ss}}(\mathrm{D}, R)$, we let $[\Phi, U]$ denote its equivalence class.

- $\left[\operatorname{Rep}_{\mathrm{ss}}(\mathrm{D}, R)\right]=\operatorname{Rep}_{\mathrm{ss}}(\mathrm{D}, R) / \sim$.

- $\left[\operatorname{Irr}_{\mathrm{ss}}(\mathrm{D}, R)\right]=\operatorname{Irr}_{\mathrm{ss}}(\mathrm{D}, R) / \sim$.

- $\left[\operatorname{Indec}_{\mathrm{ss}}(\mathrm{D}, R)\right]=\operatorname{Indec}_{\mathrm{ss}}(\mathrm{D}, R) / \sim$.

- $\left[\operatorname{Nilp}_{\mathrm{ss}}(\mathrm{D}, R)\right]=\mathrm{Nilp}_{\mathrm{ss}}(\mathrm{D}, R) / \sim$.

Let $[\Phi, U],\left[\Phi^{\prime}, U^{\prime}\right] \in\left[\operatorname{Rep}_{\mathrm{sS}}(\mathrm{D}, R)\right]$, then the equivalence class of the direct sum and dual Deligne $R$-representations are independent of choice of representatives, so we let

$$
\begin{aligned}
{[\Phi, U] \oplus\left[\Phi^{\prime}, U^{\prime}\right] } & =\left[(\Phi, U) \oplus\left(\Phi^{\prime}, U^{\prime}\right)\right] \\
{[\Phi, U]^{\vee} } & =\left[(\Phi, U)^{\vee}\right]
\end{aligned}
$$

giving a well defined direct sum and dual on $\left[\operatorname{Rep}_{\mathrm{ss}}(\mathrm{D}, R)\right]$.

In the $\ell$-adic case, we recall that $\operatorname{Rep}_{\mathrm{ss}}\left(\mathrm{D}, \overline{\mathbb{Q}_{\ell}}\right)=\operatorname{Nilp}_{\mathrm{ss}}\left(\mathrm{D}, \overline{\mathbb{Q}_{\ell}}\right)$, hence the following proposition shows that in this case one gains nothing by introducing the equivalence relation $\sim$.

Proposition 4.9. The canonical surjection from $\operatorname{Nilp}_{\mathrm{ss}}(\mathrm{D}, R)$ onto $\left[\operatorname{Nilp}_{\mathrm{ss}}(\mathrm{D}, R)\right]$ is the identity, we write:

$$
\left[\operatorname{Nilp}_{\mathrm{ss}}(\mathrm{D}, R)\right]=\operatorname{Nilp}_{\mathrm{ss}}(\mathrm{D}, R)
$$

Proof. Take $(\Phi, N)$ a Deligne representation with $N$ nilpotent, it is enough to prove that $(\Phi, N)$ and $(\Phi, \lambda N)$ are isomorphic if $\lambda \in R^{\times}$. Let $r$ be the nilpotency index of $N$. As the iterated kernels $\operatorname{Ker}\left(N^{k}\right)$ are all $\mathrm{W}_{F}$-stable, we can construct a $\mathrm{W}_{F}$-stable complement $Q_{r-1}$ of $\operatorname{Ker}\left(N^{r-1}\right)$ in $\operatorname{Ker}\left(N^{r}\right)=V_{\Phi}$. Then $N\left(Q_{r-1}\right)$ is also $\mathrm{W}_{F}$-stable, and

$$
N\left(Q_{r-1}\right) \cap \operatorname{Ker}\left(N^{r-2}\right)=\{0\} .
$$


Hence $N\left(Q_{r-1}\right) \oplus \operatorname{Ker}\left(N^{r-2}\right)$ admits a $\mathrm{W}_{F}$-stable complement $U_{r-1}$ in $\operatorname{Ker}\left(N^{r-1}\right)$, and

$$
Q_{r-2}=N\left(Q_{r-1}\right) \oplus U_{r-1}
$$

is a $\mathrm{W}_{F}$-stable complement of $\operatorname{Ker}\left(N^{r-2}\right)$ in $\operatorname{Ker}\left(N^{r-1}\right)$ such that $N\left(Q_{r-1}\right) \subseteq Q_{r-2}$. Contin-

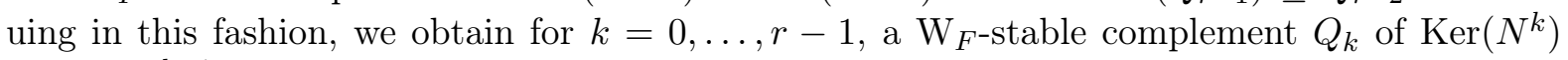
in $\operatorname{Ker}\left(N^{k+1}\right)$ such that $N\left(Q_{k}\right) \subseteq Q_{k-1}$ for $k \geqslant 1$, and

$$
V_{\Phi}=\bigoplus_{k=0}^{r-1} Q_{k} .
$$

We define $P \in \mathrm{GL}\left(V_{\Phi}\right)$ to be equal to $\lambda^{i} \operatorname{Id}_{Q_{i}}$ on $Q_{i}$. Then $P$ commutes with $\Phi(w)$ for all $w \in \mathrm{W}_{F}$, and as is checked on each $Q_{i}$, one has $P \lambda N=N P$. Hence $P$ defines the required isomorphism.

Corollary 4.10. We have $\left[\operatorname{Rep}_{\mathrm{ss}}\left(\mathrm{D}, \overline{\mathbb{Q}_{\ell}}\right)\right]=\operatorname{Nilp}_{\mathrm{ss}}\left(\mathrm{D}, \overline{\mathbb{Q}_{\ell}}\right)$.

In particular, for $[\Phi, U],\left[\Phi^{\prime}, U^{\prime}\right]$ in $\left[\operatorname{Rep}_{\mathrm{ss}}\left(\mathrm{D}, \overline{\mathbb{Q}_{\ell}}\right)\right]$, the equivalence class $\left[(\Phi, U) \otimes\left(\Phi^{\prime}, U^{\prime}\right)\right]$ is independent of the choice of representatives for $[\Phi, U],\left[\Phi^{\prime}, U^{\prime}\right]$ (we can in fact remove the brackets everywhere so the assertion is obvious). This is not true anymore when $R=\overline{\mathbb{F}_{\ell}}$, first we already saw in Example 3.11 that $\mathrm{W}_{F}$-semisimplicity is not preserved, but even if it is, $[\Phi, U] \otimes\left[\Phi^{\prime}, U^{\prime}\right]$ is still not well-defined in general. However there is a also a natural solution to this problem as we shall see in Section 4.4. We give an example:

Example 4.11. Take $\ell \neq 2, \Phi=\mathbf{1} \in \operatorname{Irr}\left(\mathrm{W}_{F}, \overline{\mathbb{F}_{\ell}}\right)$ and suppose that $q \equiv 1[\ell]$. Then $(\mathbf{1}, \mathrm{Id}) \sim$ $(\mathbf{1},-\mathrm{Id})$, but

$$
(\mathbf{1}, \mathrm{Id}) \otimes(\mathbf{1}, \mathrm{Id})=(\mathbf{1}, 2 \mathrm{Id}), \text { whereas }(\mathbf{1}, \mathrm{Id}) \otimes(\mathbf{1},-\mathrm{Id})=(\mathbf{1}, 0),
$$

which are inequivalent. However, for all pairs $(\lambda, \mu) \in\left(\overline{\mathbb{F}}_{\ell} \times\right)^{2}$ outside the hyperplane of $\left(\overline{\mathbb{F}_{\ell}}\right)^{2}$ defined by $\mu+\lambda=0$, we have $[(\mathbf{1}, \lambda \mathrm{Id}) \otimes(\mathbf{1}, \mu \mathrm{Id})]=[\mathbf{1}, \mathrm{Id}]$, and we shall set

$$
[1, I d] \otimes[1, I d]=[1, I d]
$$

There are two cases where the tensor product behaves well which will prove fundamental later:

Lemma 4.12. Let $(\Phi, U),\left(\Phi^{\prime}, U^{\prime}\right) \in \operatorname{Rep}_{\mathrm{ss}}\left(\mathrm{D}, \overline{\mathbb{F}_{\ell}}\right)$.

(1) If $\Phi$ is a direct sum of characters, then $(\Phi, U) \otimes\left(\Phi^{\prime}, U^{\prime}\right)$ is $\mathrm{W}_{F^{-} \text {-semisimple. }}$

(2) If moreover $U=N$ is nilpotent, and if $\left(\Phi_{1}, U_{1}\right) \sim(\Phi, U)$ and $\left(\Phi_{1}^{\prime}, U_{1}^{\prime}\right) \sim\left(\Phi^{\prime}, U^{\prime}\right)$, then

$$
\left(\Phi_{1}, U_{1}\right) \otimes\left(\Phi_{1}^{\prime}, U_{1}^{\prime}\right) \sim(\Phi, U) \otimes\left(\Phi^{\prime}, U^{\prime}\right) .
$$

Proof. Tensoring by a character preserves simplicity hence (1). For (2), it is enough to treat the case where $\left(\Phi^{\prime}, U^{\prime}\right)$ is indecomposable. Take $\left(\Phi_{1}, U_{1}\right) \sim(\Phi, U)$ and $\left(\Phi_{1}^{\prime}, U_{1}^{\prime}\right) \sim\left(\Phi^{\prime}, U^{\prime}\right)$, then $\left(\Phi_{1}, U_{1}\right)=(\Phi, U)$ as $U$ is nilpotent, and $\left(\Phi_{1}^{\prime}, U_{1}^{\prime}\right)=\left(\Phi^{\prime}, \lambda U^{\prime}\right)$. This implies

$$
\left(\Phi_{1}, U_{1}\right) \otimes\left(\Phi_{1}^{\prime}, U_{1}^{\prime}\right)=(\Phi, U) \otimes\left(\Phi^{\prime}, \lambda U^{\prime}\right) \sim\left(\Phi, \lambda^{-1} U\right) \otimes\left(\Phi^{\prime}, U^{\prime}\right)=(\Phi, U) \otimes\left(\Phi^{\prime}, U^{\prime}\right)
$$

because $U$ is nilpotent. 
In the situation of the lemma we define

$$
[\Phi, U] \otimes\left[\Phi^{\prime}, U^{\prime}\right]=\left[(\Phi, U) \otimes\left(\Phi^{\prime}, U^{\prime}\right)\right] .
$$

We introduce the following definition, which will be convenient when dealing with indecomposable objects in $\operatorname{Rep}_{\mathrm{ss}}(\mathrm{D}, R)$.

Definition 4.13. Let $\Psi \in \operatorname{Irr}\left(\mathrm{W}_{F}, R\right)$ and $(\Phi, U) \in \operatorname{Rep}_{\mathrm{ss}}(\mathrm{D}, R)$. We say that $(\Phi, U)$ is supported on $\mathbb{Z}_{\Psi}$ if $\Phi=\bigoplus_{i} \Psi_{i}$, for $\Psi_{i} \in \mathbb{Z}_{\Psi}$.

Let $(\Phi, U) \in \operatorname{Rep}_{\mathrm{ss}}(\mathrm{D}, R)$ and $\Psi \in \operatorname{Irr}\left(\mathrm{W}_{F}, R\right)$. We write $\Phi(i, \Psi)$ for its $\nu^{i} \Psi$ isotypic component which only depends on the class $i(\bmod o(\Psi))$. As $U$ belongs to $\operatorname{Hom}_{\mathrm{W}_{F}}(\nu \Phi, \Phi)$, the $\mathrm{W}_{F^{-}}$ subrepresentation $\Phi\left(\mathbb{Z}_{\Psi}\right)=\bigoplus_{i=1}^{o(\Psi)-1} \Phi(i, \Psi)$ is stable under $U$. As there is a finite subset $S$ of $\operatorname{Irr}\left(\mathrm{W}_{F}, R\right)$ such that $\Phi=\bigoplus_{\Psi \in S} \Phi\left(\mathbb{Z}_{\Psi}\right)$, we deduce the following lemma:

Lemma 4.14. If $(\Phi, U) \in \operatorname{Indec}_{\mathrm{SS}}(\mathrm{D}, R)$, then there is $\Psi \in \operatorname{Irr}\left(\mathrm{W}_{F}, R\right)$ such that $(\Phi, U)$ is supported on $\mathbb{Z}_{\Psi}$.

\subsection{Irreducible $\mathrm{W}_{F^{-} \text {-semisimple Deligne representations }}$}

We start with the following simple observation.

Lemma 4.15. If $(\Phi, U) \in \operatorname{Irr}_{\mathrm{ss}}(\mathrm{D}, R)$, then either $U$ is bijective, or $U$ is zero.

Proof. Clear, as $\operatorname{Ker}(U)$ is a $(\Phi, U)$-stable subspace.

An irreducible Deligne representation $(\Phi, 0)$ is nothing more than an irreducible representation of $\mathrm{W}_{F}$. In particular, when $R=\overline{\mathbb{Q}_{\ell}}$, the map $\Phi \mapsto(\Phi, 0)$ is a bijection between $\operatorname{Irr}\left(\mathrm{W}_{F}, \overline{\mathbb{Q}_{\ell}}\right)$ and $\operatorname{Irr}_{\mathrm{ss}}\left(\mathrm{D}, \overline{\mathbb{Q}_{\ell}}\right)$ by Remark 4.2 . When $R=\overline{\mathbb{F}_{\ell}}$, we record this as a lemma:

Lemma 4.16. The map $\Phi \mapsto(\Phi, 0)$ is a bijection between $\operatorname{Irr}\left(\mathrm{W}_{F}, \overline{\mathbb{F}_{\ell}}\right)$ and $\operatorname{Irr}_{\mathrm{ss}}\left(\mathrm{D}, \overline{\mathbb{F}_{\ell}}\right) \cap$ $\operatorname{Nilp}_{\mathrm{ss}}\left(\mathrm{D}, \overline{\mathbb{F}_{\ell}}\right)$.

Notation 4.17. By abuse of notation, we set $\Psi=(\Psi, 0)=[\Psi, 0]$.

We now consider the case $U$ bijective, hence $R=\overline{\mathbb{F}_{\ell}}$. We first start with an example:

Proposition 4.18. Let $\Psi \in \operatorname{Irr}\left(\mathrm{W}_{F}, \overline{\mathbb{F}_{\ell}}\right)$ and $I \in \operatorname{Iso}_{F}\left(\nu^{o(\Psi)} \Psi, \Psi\right)$, and write $\mathcal{C}(\Psi, I)=$ $\left(\Phi(\Psi), \mathcal{C}_{I}\right)$ for the pair

$$
\begin{aligned}
\Phi(\Psi) & =\bigoplus_{k=0}^{o(\Psi)-1} \nu^{k} \Psi \\
\mathcal{C}_{I}\left(x_{0}, \ldots, x_{o(\Psi)-1}\right) & =\left(I\left(x_{o(\Psi)-1}\right), x_{0}, \ldots, x_{o(\Psi)-2}\right) .
\end{aligned}
$$

(1) We have $\mathcal{C}(\Psi, I) \in \operatorname{Irr}_{\mathrm{ss}}\left(\mathrm{D}, \overline{\mathbb{F}_{\ell}}\right)$.

(2) For all $k \in \mathbb{Z}, \mathcal{C}(\Psi, I)=\mathcal{C}\left(\nu^{k} \Psi, I\right)$.

(3) The equivalence class $[\mathcal{C}(\Psi, I)]$ is independent of $I \in \operatorname{Iso}_{F}\left(\nu^{o(\Psi)} \Psi, \Psi\right)$. 
Proof. (1) The endomorphism $\mathcal{C}_{I}$ belongs to $\operatorname{Hom}_{W_{F}}(\nu \Phi, \Phi)$ by definition, hence $\mathcal{C}(\Psi, I) \in$ $\operatorname{Rep}_{\mathrm{ss}}\left(\mathrm{D}, \overline{\mathbb{F}_{\ell}}\right)$. Whenever $W$ is a $\mathrm{W}_{F}$-stable subspace of $V=V_{\Phi(\Psi)}$, we set $W(i)=W(i, \Psi)$. Let $W$ be a nonzero Deligne subrepresentation of $V$. As it is $\mathrm{W}_{F}$-stable, we have

$$
W=\bigoplus_{i=0}^{o(\Psi)-1} W(i),
$$

and as $W \neq 0$, there is an $i \in\{0, \ldots, o(\Psi)-1\}$ such that $W(i) \neq 0$. Hence $W(i)=V(i)=$ $\nu^{i} \Psi$ by irreducibility of $V(i)$. But then $\mathcal{C}_{I}^{k}(W(i))=\mathcal{C}_{I}^{k}(V(i))=V(i+k[o(\Psi)]) \subseteq W$ for all $k \in \mathbb{Z}$, and $W=V$.

(2) By definition $\mathcal{C}_{I} \in \mathrm{Iso}_{\mathrm{W}}\left(\Phi(\Psi), \nu^{-1} \Phi(\Psi)\right)$ and commutes with $\mathcal{C}_{I}$.

(3) Write $\mathcal{C}(\Psi, I)=\left(\Phi(\Psi), \mathcal{C}_{I}\right)$ and take $\lambda \in \overline{\mathbb{F}}_{\ell} \times$. Set $V=V_{\Phi}$. Take $u \in \overline{\mathbb{F}_{\ell}}$ such that $u^{o(\Psi)}=\lambda$. Define $A \in \operatorname{End}(V)$ by the formula

$$
A\left(x_{0}, x_{1}, x_{2}, \ldots, x_{o(\Psi-1)}\right)=\left(x_{0}, u x_{1}, \ldots, u^{o(\Psi)-2} x_{o(\Psi)-2}, u^{o(\Psi)-1} x_{o(\Psi)-1}\right) .
$$

Then

$$
\begin{aligned}
A \mathcal{C}_{\lambda I}\left(x_{0}, x_{1}, \ldots, x_{o(\Psi)-2}, x_{o(\Psi)-1}\right) & =A\left(\lambda I\left(x_{o(\Psi)-1}\right), x_{0}, x_{1}, \ldots, x_{o(\Psi)-2}\right) \\
& =\left(\lambda I\left(x_{o(\Psi)-1}\right), u x_{0}, u^{2} x_{1}, \ldots, u^{o(\Psi)-1} x_{o(\Psi)-2}\right) \\
& =u\left(u^{o(\Psi)-1} I\left(x_{o(\Psi)-1}\right), x_{0}, u x_{1}, \ldots, u^{o(\Psi)-2} x_{o(\Psi)-2}\right) \\
& =u \mathcal{C}_{I} A\left(x_{0}, x_{1}, \ldots, x_{o(\Psi)-2}, x_{o(\Psi)-1}\right),
\end{aligned}
$$

i.e.

$$
A \mathcal{C}_{\lambda I}=u \mathcal{C}_{I} A
$$

As $A$ commutes with $\Phi(w)$ for any $w \in \mathrm{W}_{F}$, it defines an isomorphism between the Deligne representations $\left(\Phi(\Psi), \mathcal{C}_{\lambda I}\right)$ and $\left(\Phi(\Psi), u \mathcal{C}_{I}\right)$, hence the result.

Notation 4.19. Under the notation of Proposition 4.18 and by Parts (2), (3), it thus makes sense to set

$$
\mathcal{C}\left(\mathbb{Z}_{\Psi}, I\right)=\mathcal{C}(\Psi, I), \quad \text { and } \quad \mathcal{C}\left(\mathbb{Z}_{\Psi}\right)=\left[\mathcal{C}\left(\mathbb{Z}_{\Psi}, I\right)\right]
$$

We now want to show that all elements of $\operatorname{Irr}_{\mathrm{ss}}\left(\mathrm{D}, \overline{\mathbb{F}_{\ell}}\right)$ with bijective Deligne operator are in such a class.

Let $(\Phi, U)$ be an irreducible $\mathrm{W}_{F}$-semisimple Deligne representation with $U$ bijective, and set $V=$ $V_{\Phi}$. By Lemma 4.14 , we know that $(\Phi, U)$ is supported on an irreducible line $\mathbb{Z}_{\Psi}$. Again we set $W(i)=W(i, \Psi)$ for any $\mathrm{W}_{F}$-stable subspace $W$ of $V$. We will now gather some information on the structure of $(\Phi, U)$. The first basic observation is that the relation

$$
\nu(w) U \Phi(w)=\Phi(w) U
$$

for $w \in \mathrm{W}_{F}$, can be rewritten as

$$
\left(U \nu(w) \Phi(w) U^{-1}\right) U=\Phi(w) U
$$

which shows that $U$ sends $V(i)$ to $V(i+1)$, in a necessarily bijective manner. We deduce the following lemma: 
Lemma 4.20. We have $V(i)=U^{i}(V(0))$, for $i=0, \ldots, o(\Psi)-1$. In particular all the $\mathrm{W}_{F^{-}}$ isotypic components $V(i)$ have the same dimension for $i=0, \ldots, o(\Psi)-1$.

We now give a useful description the endomorphism ring of $(\Phi, U)$. In order to do so, we fix an isomorphism of $\mathrm{W}_{F}$-modules

$$
J: V(0) \stackrel{\sim}{\rightarrow} V(0)
$$

from $\left.\nu^{o(\Psi)} \Phi\right|_{V(0)}$ to $\left.\Phi\right|_{V(0)}$. Notice that as $\left.\Phi\right|_{V(0)}$ is the direct sum of $m$-copies of $\Psi$, for some $m \geqslant$ 1 , then

$$
A_{0}=\operatorname{End}_{F}\left(\left.\Phi\right|_{V(0)}\right)
$$

is isomorphic to $\operatorname{Mat}(m, R)$. This implies that all automorphisms of $A_{0}$ are inner, in particular as $A_{0}$ is also equal (not just isomorphic) to $\operatorname{End}_{\mathrm{W}_{F}}\left(\left.\nu^{o(\Psi)} \Phi\right|_{V(0)}\right.$ ), there is $P \in A_{0}^{\times}$such that

$$
J^{-1} A J=P^{-1} A P,
$$

for all $A \in A_{0}$. We fix such a $P$ and put

$$
Y=\left(\left.U^{o(\Psi)}\right|_{V(0)}\right) J P^{-1} \in A_{0}
$$

Proposition 4.21. Under the notation above, the endomorphism algebra $\operatorname{End}_{\mathrm{D}}(V)$ is isomorphic to the algebra $C_{A_{0}}(Y)$ of elements of $A_{0}$ commuting with $Y$. An isomorphism is given by

$$
\begin{aligned}
C_{A_{0}}(Y) & \rightarrow \operatorname{End}_{\mathrm{D}}(V) \\
L_{0} & \mapsto L=L_{0} \oplus \cdots \oplus L_{o(\Psi)-1},
\end{aligned}
$$

where $L_{i}=U^{i} L_{0} U^{-i} \in \operatorname{End}_{\mathrm{W}_{F}}\left(\left.\Phi\right|_{V(i)}\right)$.

Proof. Take $L \in \operatorname{End}_{\mathrm{D}}(V)$, then $L$ stabilizes each $V(i)$ as it commutes with the action of $\mathrm{W}_{F}$, and we denote by $L_{i}$ the restriction $\left.L\right|_{V(i)}$. Because $L$ commutes with $U$ we have

$$
L_{i}=U^{i} L_{0} U^{-i} \in \operatorname{End}_{F}\left(\left.\Phi\right|_{V(i)}\right),
$$

and moreover $L_{0}$ must commute with $U_{0}=\left.\left(U^{o(\Psi)}\right)\right|_{V(0)}$. Notice that $U_{0} \in \operatorname{End}_{\overline{\mathbb{F}_{\ell}}}(V(0))$ but has no reason to belong to $A_{0}$.

Conversely given $L_{0} \in A_{0}$ commuting with $U_{0}$, the map

$$
L=L_{0} \oplus \cdots \oplus L_{o(\Psi)-1}
$$

with $L_{i}=U^{i} L_{0} U^{-i}$ belongs to $\operatorname{End}_{\mathrm{D}}(V)$. Hence we have an isomorphism from the subalgebra

$$
B_{0}=\left\{L_{0} \in A_{0}, L_{0} U_{0}=U_{0} L_{0}\right\}
$$

of $A_{0}$ to $\operatorname{End}_{\mathrm{D}}(V)$ defined by $L_{0} \mapsto L$. Now the map $X=U_{0} J$ belongs to $A_{0}$, hence the relation

$$
L_{0} U_{0}=U_{0} L_{0}
$$

is equivalent to

$$
\begin{gathered}
L_{0} U_{0}=U_{0} J J^{-1} L_{0} \Leftrightarrow L_{0} X J^{-1} \\
=X J^{-1} L_{0} \Leftrightarrow L_{0} X=X J^{-1} L_{0} J \Leftrightarrow L_{0} X=X P^{-1} L_{0} P \\
\Leftrightarrow L_{0} X P^{-1}=X P^{-1} L_{0} .
\end{gathered}
$$

This ends the proof. 
Let $F$ be a field, $X \in \operatorname{Mat}(n, F)$, and set

$$
C_{\operatorname{Mat}(n, F)}(X)=\{Y \in \operatorname{Mat}(n, F), Y X=X Y\},
$$

the subalgebra of matrices commuting with $X$. For use in the next proof, we recall the following lemma from basic linear algebra:

Lemma 4.22. The algebra $C_{\operatorname{Mat}(n, F)}(X)$ has dimension 1 if and only if $n=1$.

Proof. Suppose that $C_{\operatorname{Mat}(n, F)}(X)$ has dimension one, then in particular $F[B] \simeq F[X] /\left(f_{B}\right)$ where $f_{B}$ is the minimal polynomial of $B$ must be one dimensional. Hence $f_{B}$ has degree 1 and $B$ is a multiple of $I_{n}$. The statement follows.

A corollary of Proposition 4.21 and Lemma 4.22 is the following:

Corollary 4.23. We have $\Phi(i) \simeq \nu^{i} \Psi$ for $i=0, \ldots, o(\Psi)-1$.

Proof. By Schur's lemma, the ring $\operatorname{End}_{\mathrm{D}}\left(V_{\Phi}\right)$ is one dimensional, hence by Proposition 4.21, the algebra $C_{A_{0}}(Y)$ is one-dimensional. However, as $A_{0} \simeq \operatorname{Mat}\left(m, \overline{\mathbb{F}_{\ell}}\right)$, if

$$
\Phi=\underbrace{\Psi \oplus \cdots \oplus \Psi}_{m \times}
$$

Lemma 4.22 implies that $m=1$ i.e. $\Phi(0) \simeq \Psi$, and $\Phi(i) \simeq \nu^{i} \Psi$ by Lemma 4.20

We obtain the main result of this section.

Theorem 4.24. Take $(\Phi, U) \in \operatorname{Irr}_{\mathrm{ss}}\left(\mathrm{D}, \overline{\mathbb{F}_{\ell}}\right)$ with $U$ bijective, then there is a unique irreducible line $\mathbb{Z}_{\Psi}$ of $\mathrm{W}_{F}$ such that

$$
[\Phi, U]=\mathcal{C}\left(\mathbb{Z}_{\Psi}\right)
$$

Proof. We already explained that $(\Phi, U)$ is supported on an irreducible line $\mathbb{Z}_{\Psi}$, which is necessarily unique by uniqueness of the decomposition of $\Phi$ into a direct sum of elements in $\operatorname{Irr}\left(\mathrm{W}_{F}, \overline{\mathbb{F}_{\ell}}\right)$. Set $V=V_{\Phi}$ and $V(i)$ its $\nu^{i} \Psi$-isotypic component. Take $I \in \operatorname{Iso}_{F}(\nu \Psi, \Psi)$ and consider $\mathcal{C}(\Psi, I)=\left(\Phi(\Psi), \mathcal{C}_{I}\right)$. By Corollary 4.23, $V(i) \simeq \nu^{i} \Psi$. Let $J_{0}$ be an isomorphism between $V(0)$ and $\Psi$, then for each $i$ between 0 and $o(\Psi)-1$, the map $J_{i}=\mathcal{C}_{I}^{i} J_{0} U^{-i}$ is a linear bijection from $V(i)$ to $\nu^{i} \Psi$ commuting with the action of $\mathrm{W}_{F}$. Hence the map $J=\bigoplus_{i=0}^{o(\Psi)-1} J_{i}$ is a linear bijection from $V$ to $\Phi(\Psi)$ commuting with the action of $\mathrm{W}_{F}$. It intertwines the action of $U$ and $\mathcal{C}_{I}$ on each $V(i)$ : for $i=0, \ldots, o(\Psi)-2$, we have

$$
\left.J U\right|_{V(i)}=\left.\mathcal{C}_{I} J\right|_{V(i)}=\left.\mathcal{C}_{\mu I} J\right|_{V(i)}
$$

for any $\mu \in{\overline{\mathbb{F}_{\ell}}}^{\times}$. On $V(o(\Psi)-1)$, by Schur's lemma, there is $\lambda \in \overline{\mathbb{F}}_{\ell} \times$ such that

$$
\left.J U\right|_{V(o(\Psi)-1)}=\left.\lambda \mathcal{C}_{I} J\right|_{V(o(\Psi)-1)}=\left.\mathcal{C}_{\lambda I} J\right|_{V(o(\Psi)-1)} .
$$

This shows that $J$ is an isomorphism between $(\Phi, U)$ and $\mathcal{C}(\Psi, \lambda I)$. 


\subsection{Indecomposable $\mathrm{W}_{F}$-semisimple Deligne representations}

Take $(\Phi, U) \in \operatorname{Indec}_{\mathrm{sS}}(\mathrm{D}, R)$, in particular it is supported on $\mathbb{Z}_{\Psi}$ for $\Psi$ an irreducible representation of $\mathrm{W}_{F}$. As before we write $V=V_{\Phi}$ and $U=S+N$, and we already observed that $U=N$ when $R=\overline{\mathbb{Q}_{\ell}}$. We consider $R=\overline{\mathbb{F}_{\ell}}$ for a moment, the Deligne relation satisfied by $U$ implies that the nonzero eigenvalues of $S$ can be partitioned into orbits of $q^{\mathbb{Z}}=q^{\mathbb{Z} / o(\nu) \mathbb{Z}} \simeq \mathbb{Z} / o(\nu) \mathbb{Z}$ acting by multiplication.

Lemma 4.25. For $(\Phi, U) \in \operatorname{Indec}_{\mathrm{sS}}\left(\mathrm{D}, \overline{\mathbb{F}_{\ell}}\right)$, the eigenvalues of $U$ lie in a single orbit under this action of $\mathbb{Z} / o(\nu) \mathbb{Z}$, in particular $U$ is either bijective or nilpotent.

Proof. We write $U=S+N$, and as before we have

$$
\operatorname{Spec}(S)=\{0\} \sqcup O_{1} \sqcup \cdots \sqcup O_{s}
$$

as the union of the orbit of 0 and the $s$ orbits of nonzero eigenvalues. We set $V_{0}=\operatorname{Ker}(S)$, and $V_{i}=\bigoplus_{\lambda \in O_{i}} V_{\lambda}$ so that $V=\bigoplus_{i=0}^{s} V_{i}$ is a decomposition into $\mathrm{W}_{F}$-stable summands. The summands are stable under $U$ as $U$ commutes with $S$. Hence the decomposition $V=\bigoplus_{i=0}^{s} V_{i}$ is a direct sum of Deligne representations. As $(\Phi, U)$ is indecomposable, we must have $V=V_{i}$ for some $i$.

We continue with our coefficient ring $R$ denoting either $\overline{\mathbb{Q}_{\ell}}$ or $\overline{\mathbb{F}_{\ell}}$. Let us first consider the case $U$ nilpotent, and start with a classical example.

Definition 4.26. Take $m \geqslant 1$, and denote by $[0, r-1]$ the $\mathrm{W}_{F}$-semisimple Deligne representation $(\Phi(r), N(r))$ where

$$
\Phi(r)=\bigoplus_{k=0}^{r-1} \nu^{k}, \quad \text { and } \quad N(r)\left(x_{0}, \ldots, x_{r-1}\right)=\left(0, x_{0}, \ldots, x_{r-2}\right) .
$$

For $a \leqslant b$ in $\mathbb{Z}$, we set $[a, b]=\nu^{a}[0, b-a]$. By convention if $b<a$, we set $[a, b]$ to be the null vector space.

Lemma 4.27. We have $[0, r-1] \in \operatorname{Indec}_{\mathrm{ss}}(\mathrm{D}, R)$.

Proof. In fact it is already indecomposable as an $R[N(r)]$-module. Indeed, the endomorphism algebra $\operatorname{End}_{R[N(r)]}\left(V_{[0, r-1]}\right)$ is equal to $R[N(r)]$ because $N(r)$ is a cyclic endomorphism of $V_{[0, r-1]}$. Now the ring $R[N(r)]=R[X] /\left(X^{r}\right)$ is local, so the $R[N(r)]$-module $V_{[0, r-1]}$ is indecomposable.

More generally, we have:

Proposition 4.28. Take $\Psi \in \operatorname{Irr}\left(\mathrm{W}_{F}, R\right)=\operatorname{Irr}_{\mathrm{ss}}(\mathrm{D}, R) \cap \operatorname{Nilp}_{s s}(\mathrm{D}, R)$ and $r \geqslant 1$, then

$$
[0, r-1] \otimes \Psi \in \operatorname{Indec}_{\mathrm{ss}}(\mathrm{D}, R) .
$$

Moreover, take $N(r)$ as in Definition 4.26 . If $R=\overline{\mathbb{F}_{\ell}}$, and $I \in \operatorname{Iso}_{F}\left(\nu^{o(\Psi)} \Psi, \Psi\right)$, then

$$
\operatorname{End}_{\mathrm{D}}([0, r-1] \otimes \Psi)=\overline{\mathbb{F}_{\ell}}\left[N(r)^{o(\Psi)} \otimes I^{-1}\right] .
$$

If $R=\overline{\mathbb{Q}_{\ell}}$, then

$$
\operatorname{End}_{D}([0, r-1] \otimes \Psi) \simeq \overline{\mathbb{Q}_{\ell}} .
$$

In both cases they are local rings. 
Proof. First, by Lemma 4.12, $[0, r-1] \otimes \Psi \in \operatorname{Rep}_{\mathrm{ss}}(\mathrm{D}, R)$. Let's compute the endomorphism algebra of $[0, r-1] \otimes \Psi$. We do the case $R=\overline{\mathbb{F}_{\ell}}$, the case $R=\overline{\mathbb{Q}_{\ell}}$ being similar. One has

$$
\operatorname{End}_{\overline{\mathbb{F}_{\ell}}}([0, r-1] \otimes \Psi)=\operatorname{End}_{\overline{\mathbb{F}_{\ell}}}([0, r-1]) \otimes \operatorname{End}_{\overline{\mathbb{F}_{\ell}}}(\Psi) .
$$

The Deligne operator $N(\Psi, r)$ of $[0, r-1] \otimes \Psi \in \operatorname{Indec}_{\mathrm{ss}}(\mathrm{D}, R)$ is $N(r) \otimes \mathrm{Id}$, hence

$$
\operatorname{End}_{\overline{\mathbb{F}_{\ell}}[N(\Psi, r)]}([0, r-1] \otimes \Psi)=\operatorname{End}_{\overline{\mathbb{F}_{\ell}}[N(r)]}([0, r-1]) \otimes \operatorname{End}_{\overline{\mathbb{F}_{\ell}}}(\Psi)=\overline{\mathbb{F}_{\ell}}[N(r)] \otimes \operatorname{End}_{\overline{\mathbb{F}_{\ell}}}(\Psi) .
$$

Fixing $I \in \operatorname{Hom}\left(\nu^{(o(\Psi)} \Psi, \Psi\right)$, and using the basis Id, $N(r), \ldots, N(r)^{r-1}$ of $\overline{\mathbb{F}_{\ell}}[N]$, one checks that the subalgebra of $\overline{\mathbb{F}_{\ell}}[N(r)] \otimes \operatorname{End}_{\overline{\mathbb{F}_{\ell}}}(\Psi)$ commuting with the action of $\mathrm{W}_{F}$ is $\mathbb{F}_{l}\left[N(r)^{o(\Psi)} \otimes I^{-1}\right]$, i.e.

$$
\operatorname{End}_{\mathrm{D}}([0, r-1] \otimes \Psi)=\overline{\mathbb{F}_{\ell}}\left[N(r)^{o(\Psi)} \otimes I^{-1}\right]
$$

When $R=\overline{\mathbb{Q}_{\ell}}$ we find

$$
\operatorname{End}_{\mathrm{D}}([0, r-1] \otimes \Psi) \simeq \overline{\mathbb{Q}_{\ell}} .
$$

In both cases, these algebras are local as they are of the form $R[X] /\left(X^{l}\right)$ for $l \geqslant 0$.

Now we check that these are the only indecomposable $\mathrm{W}_{F}$-semisimple Deligne representations with nilpotent Deligne operator. If $N$ is a nilpotent endomorphism of an $R$-vector space, we denote by $\operatorname{ind}(N)$ its nilpotency index.

Theorem 4.29. Let $(\Phi, N) \in \operatorname{Indec}_{\mathrm{ss}}(\mathrm{D}, R)$ with $N$ nilpotent, then there is a unique $\Psi \in$ $\operatorname{Irr}\left(\mathrm{W}_{F}, R\right)$ and a unique $r \geqslant 1$ such that

$$
[\Phi, N]=(\Phi, N)=[0, r-1] \otimes \Psi=[0, r-1] \otimes[\Psi] .
$$

Proof. The uniqueness of $\Psi$ (up to isomorphism or equivalence as these notions agree for nilpotent Deligne representations) and $r$ are clear. Set $r=\operatorname{ind}(N)$ and $V=V_{\Phi}$. As in the proof of Proposition 4.9, we construct $\mathrm{W}_{F}$-stable subspaces $S_{i}$ such that $V=\bigoplus_{i=0}^{r-1} S_{i}$ and $N\left(S_{i}\right) \subseteq S_{i-1}$. Suppose one $S_{r-i}$ has length $\geqslant 2$ as a $\mathrm{W}_{F}$-module and take $i=i_{0}$ minimal for this property. Write

$$
S_{r-i_{0}}=\Psi_{r-i_{0}} \oplus U_{r-i_{0}}
$$

with $\Psi_{r-i_{0}} \in \operatorname{Irr}\left(\mathrm{W}_{F}, R\right)$ and $U_{r-i_{0}}$ stable under $\mathrm{W}_{F}$, then take a complement $Z_{r-i_{0}-1}$ of

$$
N\left(\Psi_{r-i_{0}}\right) \oplus N\left(U_{r-i_{0}}\right)
$$

in $S_{r-i_{0}-1}$, set

$$
U_{r-i_{0}-1}=N\left(U_{r-i_{0}}\right) \oplus Z_{r-i_{0}-1}
$$

and continue. We construct a nontrivial $(\Phi, N)$-stable decomposition

$$
\left[S_{r-1} \oplus \cdots \oplus S_{r-\left(i_{0}-1\right)} \oplus \Psi_{r-i_{0}} \oplus \cdots \oplus N^{r-i_{0}}\left(\Psi_{r-i_{0}}\right)\right] \oplus\left[U_{r-i_{0}} \oplus U_{r-i_{0}-1} \oplus \cdots \oplus U_{0}\right]
$$

of $V$, a contradiction. Hence each $S_{r-i}$ is an irreducible representation of $\mathrm{W}_{F}$. It then suffices to choose $\Psi=S_{r-1}$.

It remains to consider the indecomposable $\mathrm{W}_{F}$-semisimple Deligne representations $(\Phi, U)$ with $U$ invertible, in particular $R=\overline{\mathbb{F}_{\ell}}$. We begin with an example. 
Proposition 4.30. Let $\Psi \in \operatorname{Irr}\left(\mathrm{W}_{F}, \overline{\mathbb{F}_{\ell}}\right), I \in \operatorname{IsoW}_{F}\left(\nu^{o(\Psi)} \Psi, \Psi\right)$, and $r \geqslant 1$, then

$$
[0, r-1] \otimes \mathcal{C}\left(\mathbb{Z}_{\Psi}, I\right) \in \operatorname{Indec}_{\mathrm{ss}}\left(\mathrm{D}, \overline{\mathbb{F}_{\ell}}\right),
$$

and its Deligne operator $U(r, I)$ is bijective with Jordan decomposition $U(r, I)=S(r, I)+N(r, I)$

$$
S(r, I)=\operatorname{Id} \otimes \mathcal{C}_{I}, \quad \text { and } \quad N(r, I)=N(r) \otimes \mathrm{Id} .
$$

Its endomorphism algebra is the local ring

$$
\operatorname{End}_{\mathrm{D}}\left([0, r-1] \otimes \mathcal{C}\left(\mathbb{Z}_{\Psi}, I\right)\right)=\overline{\mathbb{F}_{\ell}}\left[N(r) \otimes \mathcal{C}_{I}^{-1}\right]=\overline{\mathbb{F}_{\ell}}\left[N(r, I) \circ S(r, I)^{-1}\right] .
$$

Proof. First notice that $[0, r-1] \otimes \mathcal{C}\left(\mathbb{Z}_{\Psi}, I\right)$ is indeed $\mathrm{W}_{F}$-semisimple thanks to Lemma 4.12 . Set $S=S(r, I)=\operatorname{Id} \otimes \mathcal{C}_{I}$ and $N=N(r, I)=N(r) \otimes \operatorname{Id}$. Then $S$ and $N$ clearly commute, $N$ is nilpotent and $S$ is semisimple because $\mathcal{C}_{I}$ is $\left(\mathcal{C}_{I}^{o(\nu)}\right.$ is a nonzero scalar $\mu$, and $X^{o(\nu)}-\mu$ has simple roots because $o(\nu)$ is prime to $\ell$ ). Thus $U=S+N$ is bijective because $S$ is, hence it remains to check that $[0, r-1] \otimes \mathcal{C}\left(\mathbb{Z}_{\Psi}, I\right)$ is indecomposable, and we do this by looking at the endomorphism algebra again. We recall that the commutant of $U$ is that of $S$ intersected with that of $N$. The commutant of $N$ in $\operatorname{End}_{\overline{\mathbb{F}_{\ell}}}\left([0, r-1] \otimes \mathcal{C}\left(\mathbb{Z}_{\Psi}, I\right)\right)$ is $\overline{\mathbb{F}_{\ell}}[N] \otimes \operatorname{End}_{\overline{\mathbb{F}_{\ell}}}\left(\mathcal{C}\left(\mathbb{Z}_{\Psi}, I\right)\right)$. Hence we need to look at the commutant of the joint action of $\mathrm{W}_{F}$ and $S$ inside $\overline{\mathbb{F}_{\ell}}[N] \otimes \operatorname{End}_{\overline{\mathbb{F}_{\ell}}}\left(\mathcal{C}\left(\mathbb{Z}_{\Psi}, I\right)\right)$. Writing an element in this commutant under the form $\sum_{k=0}^{r-1} N^{k} \otimes A_{k}$, the endomorphism $A_{k}$ must belong to $\operatorname{Hom}_{\mathrm{W}_{F}}\left(\mathcal{C}\left(\mathbb{Z}_{\Psi}, I\right), \nu^{k} \mathcal{C}\left(\mathbb{Z}_{\Psi}, I\right)\right)$ and must commute with $\mathcal{C}_{I}$, i.e.

$$
\mathcal{C}_{I}^{k} A_{k} \in \operatorname{End}_{\mathrm{D}}\left(\mathcal{C}\left(\mathbb{Z}_{\Psi}, I\right)\right) \simeq \overline{\mathbb{F}_{\ell}},
$$

the latter isomorphism by Schur's lemma. Hence our element is of the form $\sum_{k=0}^{r-1} \lambda_{k} N(r)^{k} \otimes \mathcal{C}_{I}^{-k}$, and this implies that

$$
\operatorname{End}_{\mathrm{D}}\left([0, r-1] \otimes \mathcal{C}\left(\mathbb{Z}_{\Psi}, I\right)\right)=\overline{\mathbb{F}_{\ell}}\left[N(r) \otimes \mathcal{C}_{I}^{-1}\right]=\overline{\mathbb{F}_{\ell}}\left[N(r, I) \circ S(r, I)^{-1}\right] .
$$

As $N(r) \otimes \mathcal{C}_{I}^{-1}$ is nilpotent, this ring is local.

We notice as in Proposition 4.18 (3), that the equivalence class of such a Deligne representation is independent of $I$. It is a consequence of Lemma 4.12 and Proposition 4.18 (3):

Lemma 4.31. We have

$$
\left[[0, r-1] \otimes \mathcal{C}\left(\mathbb{Z}_{\Psi}, I\right)\right]=[0, r-1] \otimes\left[\mathcal{C}\left(\mathbb{Z}_{\Psi}, I\right)\right]=[0, r-1] \otimes \mathcal{C}\left(\mathbb{Z}_{\Psi}\right) .
$$

Now we move on to the description of $\left[\operatorname{Indec}_{\mathrm{ss}}\left(\mathrm{D}, \overline{\mathbb{F}_{\ell}}\right)\right]$. We know by Lemma 4.14 that $(\Phi, U) \in$ $\operatorname{Indec}_{\mathrm{ss}}\left(\mathrm{D}, \overline{\mathbb{F}_{\ell}}\right)$ is always supported on an irreducible line. For the remainder of this section, we take $(\Phi, U) \in \operatorname{Indec}_{\mathrm{SS}}\left(\mathrm{D}, \overline{\mathbb{F}_{\ell}}\right)$ supported on the irreducible line $\mathbb{Z}_{\Psi}$ with $U=S+N$ invertible.

Lemma 4.32. The Deligne representation $(\Phi, S)$ is semisimple as a Deligne representation.

Proof. Let $W$ be a $(\Phi, S)$-stable subspace of $V=V_{\Phi}$, and $V_{\lambda}$ be the eigenspace of $S$ associated to an eigenvalue $\lambda$. As $W$ is $S$-stable, it is the direct sum of the eigenspaces

$$
W=\bigoplus_{\lambda \in \operatorname{Spec}(S)} W_{\lambda}
$$


where $W_{\lambda}=W \cap V_{\lambda}$. Moreover as $W$ is $\mathrm{W}_{F}$-stable, all the eigenspaces $W_{\lambda}$ for $\lambda$ in an orbit $O_{\mu}=$ $\left\{q^{\mathbb{Z}} \mu\right\}$ have the same dimension, as $\Phi\left(\right.$ Frob) sends $W_{\lambda}$ to $W_{q^{-1} \lambda}$. Hence the direct sum $W(\mu)=$ $\bigoplus_{\lambda \in O_{\mu}} W_{\lambda}$ is $W_{F}$-stable thanks to the Weil-Deligne relation, and we write

$$
W=\bigoplus_{i=1}^{r} W\left(\mu_{i}\right) .
$$

For each $\mu_{i}$, take a complement $W_{\mu_{i}}^{\prime}$ of $W_{\mu_{i}}$ inside $V_{\mu_{i}}$, so that $W_{q^{-} k \mu_{i}}^{\prime}=\Phi(\operatorname{Frob})^{k}\left(W_{\mu_{i}}^{\prime}\right)$ is a complement of $W_{q^{-k} \mu_{i}}$ inside $V_{q^{-k} \mu_{i}}$. Then we set

$$
W^{\prime}\left(\mu_{i}\right)=\bigoplus_{\lambda \in O_{\mu_{i}}} W_{\lambda}^{\prime}
$$

and we set

$$
W^{\prime}=\bigoplus_{i=1}^{r} W^{\prime}\left(\mu_{i}\right)
$$

As each $W^{\prime}\left(\mu_{i}\right)$ is $\mathrm{W}_{F}$-stable, so is $W^{\prime}$, and as $W^{\prime}$ is a direct sum of subspaces of the eigenspaces $V_{\lambda}$, the space $W^{\prime}$ is also $S$-stable. To conclude, notice that $V=W \oplus W^{\prime}$ by construction.

Now we notice that $(\Phi, S)$ is isotypic, i.e. it is the direct sum of isomorphic (not only equivalent) irreducible Weil-Deligne representations:

Lemma 4.33. There is $I \in \operatorname{Iso}_{F}\left(\nu^{o(\Psi)} \Psi, \Psi\right)$ such that $(\Phi, S)$ is isotypic of type $\mathcal{C}\left(\mathbb{Z}_{\Psi}, I\right)$.

Proof. Set $N_{S}=N S^{-1} \in \operatorname{End}_{F}(\Phi)$, and as $N$ commutes with $S$, in fact $N_{S} \in \operatorname{End}_{\mathrm{D}}((\Phi, S))$. Now $(\Phi, S)$ is the direct sum of its isotypic components of type $\mathcal{C}\left(\mathbb{Z}_{\Psi}, I_{k}\right)$ for

$$
I_{k} \in \operatorname{Iso}_{F}\left(\nu^{o(\Psi)} \Psi, \Psi\right)
$$

thanks to Lemma 4.32 and Theorem 4.24. As $N_{S}$ belongs to $\operatorname{End}_{\mathrm{D}}((\Phi, S))$, it stabilizes each of these and so does $S$ by definition of the isotypic components, so they are in fact stable under $U=S\left(I d+N_{S}\right)$. As $(\Phi, U)$ is indecomposable there is only one of them.

Using this lemma, we can obtain more information about the structure of indecomposable Deligne representations:

Put $N_{S}=N S^{-1}$ as in the proof of Lemma 4.33, and take the model $\mathcal{C}(\Psi, I)=\left(\Phi(\Psi), \mathcal{C}_{I}\right)$ for the type $\mathcal{C}\left(\mathbb{Z}_{\Psi}, I\right)$ of the isotypic Deligne representation $(\Phi, S)$. Define

$$
H=\operatorname{Hom}_{\mathrm{D}}(\mathcal{C}(\Psi, I),(\Phi, S)) .
$$

We then define a Deligne representation structure $\left(\Phi_{0}, U_{0}=S_{0}+N_{0}\right)$ on $H \otimes_{\overline{\mathbb{F}_{\ell}}} V_{\Phi(\Psi)}$ as follows:

$$
\Phi_{0}(w)=\operatorname{Id} \otimes \Phi(\Psi)(w)
$$

for $w \in \mathrm{W}_{F}$,

$$
S_{0}=\operatorname{Id} \otimes \mathcal{C}_{I}
$$

and

$$
N_{0}(h \otimes v)=N h \mathcal{C}_{I}^{-1} \otimes \mathcal{C}_{I} \cdot v=N_{S} h \otimes \mathcal{C}_{I} \cdot v .
$$

Proposition 4.34. The Deligne representations $(\Phi, U)$ and $\left(\Phi_{0}, U_{0}\right)$ are isomorphic. In particular setting $m=\operatorname{ind}(N)=\operatorname{ind}\left(N_{S}\right)$, we have $m=d_{\Phi} / d_{\Psi} o(\Psi)$. 
Proof. We introduce the map $\alpha: H \otimes_{\overline{F_{\ell}}} V_{\mathcal{C}(\Psi, I)} \rightarrow V$ defined by $\alpha(\psi \otimes v)=\psi(v)$. One checks that $\alpha$ intertwines the action of $\mathrm{W}_{F}$, of $N$ and $N_{0}$ and of $S$ and $S_{0}$. It is surjective by Lemma 4.33 . By Schur's lemma, $H$ has dimension $d_{\Phi} / d_{\Psi} o(\Psi)$, hence both spaces have the same dimension, so $\alpha$ is bijective.

We have now reached the following classification:

Theorem 4.35. Take $(\Phi, U) \in \operatorname{Indec}_{\mathrm{sS}}\left(\mathrm{D}, \overline{\mathbb{F}_{\ell}}\right)$ with $U$ bijective, there is a unique irreducible line $\mathbb{Z}_{\Psi}$ and a unique $r \geqslant 1$ such that

$$
[\Phi, U]=[0, r-1] \otimes \mathcal{C}\left(\mathbb{Z}_{\Psi}\right)
$$

Proof. Set $r=d_{\Phi} / d_{\Psi} o(\Psi)$. We define $\left(\Phi_{0}, U_{0}\right)$ as in Proposition 4.34 , it is enough to show that

$$
\left[\Phi_{0}, U_{0}\right]=[0, r-1] \otimes \mathcal{C}\left(\mathbb{Z}_{\Psi}\right)
$$

Write $V_{[0, r-1]}=\operatorname{Vect}_{\overline{\mathbb{F}_{\ell}}}\left(e_{0}, \ldots, e_{r-1}\right)$ with $e_{i}=N(r)^{i}\left(e_{0}\right)$, and $\mathrm{W}_{F}$ acting on $S_{i}=\operatorname{Vect}_{\overline{\mathbb{F}_{\ell}}}\left(e_{i}\right)$ as $\nu^{i}$. Similarly write $H=\operatorname{Vect}_{\overline{\mathbb{F}_{\ell}}}\left(h_{0}, \ldots, h_{r-1}\right)$ with $h_{i}=N_{S}^{i}\left(h_{0}\right)$. Denote by $B$ the $\overline{\mathbb{F}_{\ell}}$-linear isomorphism sending $V_{[0, r-1]} \otimes V_{\Phi}$ to $H \otimes V_{\Phi}$ defined by

$$
B\left(\sum_{i=0}^{r-1} e_{i} \otimes v_{i}\right)=\sum_{i=1}^{r-1} h_{i} \otimes \mathcal{C}_{I}^{i} v_{i} .
$$

Then

$$
B \in \operatorname{Iso}_{\mathrm{D}}\left([0, r-1] \otimes \mathcal{C}(\Psi, I),\left(\Phi_{0}, U_{0}\right)\right) .
$$

The uniqueness of $r$ and $\mathbb{Z}_{\Psi}$ are immediate.

\subsection{Tensor product for $\mathrm{W}_{F}$-semisimple Deligne representations}

We already saw the two problems of the tensor product in our setting: by Example 3.11 (3) it does not preserve $\mathrm{W}_{F}$-semisimplicity, and by Example 4.11 even when it does it does not preserve equivalence classes. In this section we take care of these two problems. These problems occur only when $R=\overline{\mathbb{F}_{\ell}}$ (see the beginning of Section 3.4 and Remark 4.12 , so for this section $R=\overline{\mathbb{F}_{\ell}}$.

First we define the $\mathrm{W}_{F}$-semisimplification $(\Phi, S)_{\mathrm{ss}}=\left(\Phi_{\mathrm{ss}}, S_{\mathrm{ss}}\right)$ of $(\Phi, S)$ when $S$ is semisimple.

Proposition 4.36. Let $(\Phi, S)$ be a Deligne $\overline{\mathbb{F}_{\ell}}$-representation with $S$ semisimple.

(1) There is a filtration

$$
\{0\}=V_{0} \subseteq V_{1} \subseteq \cdots \subseteq V_{n}=V_{\Phi}
$$

of $V_{\Phi}$ by $(\Phi, S)$-stable subspaces such that the induced Deligne $\overline{\mathbb{F}_{\ell}}$-representation $\left(\Phi_{i}, S_{i}\right)$ on $V_{i} / V_{i-1}$ is in $\operatorname{Irr}_{\mathrm{ss}}\left(\mathrm{D}, \overline{\mathbb{F}_{\ell}}\right)$.

(2) The $\mathrm{W}_{F}$-semisimple Deligne $\overline{\mathbb{F}_{\ell}}$-representation

$$
(\Phi, S)_{\mathrm{ss}}=\left(\Phi_{\mathrm{ss}}, S_{\mathrm{ss}}\right)=\bigoplus_{i=1}^{n}\left(\Phi_{i}, S_{i}\right)
$$

is independent of the filtration, and $S_{\mathrm{ss}}$ and $S$ are semisimple with the same characteristic polynomial. 
Proof. By induction, it is enough to show that $(\Phi, S)$ contains an irreducible $\mathrm{W}_{F \text {-semisimple }}$ Deligne subrepresentation. The uniqueness follows from standard facts on Jordan-Hölder composition series. Take $\Psi$ an irreducible $\mathrm{W}_{F^{-}}$-subrepresentation of $\Phi$. Then $\sum_{k \geqslant 0} \mathcal{C}^{k}(\Psi)$ is a $\mathrm{W}_{F^{-}}$ semisimple Deligne subrepresentation of $(\Phi, S)$. It thus contains an indecomposable $\mathrm{W}_{F}$-semisimple Deligne subrepresentation $\left(\Phi_{0},\left.S\right|_{\Phi_{0}}\right)$. But $\left.S\right|_{\Phi_{0}}$ being semisimple, there is $\Psi$ an irreducible representation of $\mathrm{W}_{F}$ such that $\left(\Phi_{0},\left.S\right|_{\Phi_{0}}\right)$ is either of the form $\Psi=(\Psi, 0)$ or of the form $\mathcal{C}(\Psi, I)$ for $I$ an isomorphism between $\nu^{o(\Psi)} \Psi$ and $\Psi$, thanks to Proposition 4.29 and Theorem 4.35 .

We can now define the operation $\otimes_{\mathrm{ss}}$ in $\operatorname{Rep}_{\mathrm{ss}}\left(\mathrm{D}, \mathbb{F}_{l}\right)$.

Definition 4.37. For $i=1,2$, let $\left(\Phi_{i}, U_{i}\right) \in \operatorname{Indec}_{\mathrm{ss}}\left(\mathrm{D}, \overline{\mathrm{F}_{\ell}}\right)$, and write

$$
\left(\Phi_{1}, U_{1}\right)=\left[0, r_{1}-1\right] \otimes\left(\Phi_{1}, C_{1}\right) \text { and }\left(\Phi_{2}, U_{2}\right)=\left[0, r_{2}-1\right] \otimes\left(\Phi_{2}, C_{2}\right),
$$

with $\left(\Phi_{i}, C_{i}\right) \in \operatorname{Irr}_{\mathrm{ss}}\left(\mathrm{D}, \mathbb{F}_{l}\right)$ using Proposition 4.29 and Theorem 4.35. In particular, as $C_{1}, C_{2}$ are semisimple so is $C_{1} \otimes \operatorname{Id} \oplus \operatorname{Id} \otimes C_{2}$. Hence $\left[\left(\Phi_{1}, C_{1}\right) \otimes\left(\Phi_{2}, C_{2}\right)\right]_{\mathrm{ss}}$ is well defined according to Lemma 4.36. We then set

$$
(\Phi, U) \otimes_{\mathrm{ss}}\left(\Phi^{\prime}, U^{\prime}\right)=\left[0, r_{1}-1\right] \otimes\left[0, r_{2}-1\right] \otimes\left[\left(\Phi_{1}, C_{1}\right) \otimes\left(\Phi_{2}, C_{2}\right)\right]_{\mathrm{ss}} .
$$

We extend $\otimes_{\mathrm{ss}}$ from $\operatorname{Indec}_{\mathrm{ss}}\left(\mathrm{D}, \overline{\mathbb{F}_{\ell}}\right)$ to $\operatorname{Rep}_{\mathrm{ss}}\left(\mathrm{D}, \overline{\mathbb{F}_{\ell}}\right)$ by distributivity with respect to $\oplus$.

Remark 4.38. The operation $\otimes_{\mathrm{ss}}$ is bilinear by definition, and is associative and commutative, these properties inherited from $\otimes$.

We are now ready to define $[\Phi, U] \otimes\left[\Phi^{\prime}, U^{\prime}\right]$ for any pair of Deligne representations in $\operatorname{Rep}_{\mathrm{ss}}\left(\mathrm{D}, \overline{\mathbb{F}_{\ell}}\right)$. We first consider the indecomposable case. We recall that if $U=S+N$, then $S$ and $N$ are polynomials in $U$, and that the projections onto an eigenspace of $S$ with respect to the sum of its other eigenspaces is a polynomial in $S$, hence in $U$.

Proposition 4.39. Let $(\Phi, U)$ and $\left(\Phi^{\prime}, U^{\prime}\right)$ be two $\mathrm{W}_{F}$-semisimple indecomposable Deligne representations with $U$ and $U^{\prime}$ bijective, then for all $(\lambda, \mu)$ in a subset $Z\left(U, U^{\prime}\right)$ of $\left(\overline{\mathbb{F}}_{\ell} \times\right)^{2}$ consisting of all elements outside a finite number of hyperplanes of ${\overline{F_{\ell}}}^{2}$, all representations

$$
(\Phi, \lambda U) \otimes_{\mathrm{ss}}\left(\Phi^{\prime}, \mu U^{\prime}\right)
$$

are equivalent to one another, say equivalent to $\left(\Phi, \lambda_{0} U\right) \otimes_{\mathrm{ss}}\left(\Phi^{\prime}, \mu_{0} U^{\prime}\right)$, and we set

$$
[\Phi, U] \otimes_{\mathrm{ss}}\left[\Phi^{\prime}, U^{\prime}\right]=\left[\left(\Phi, \lambda_{0} U\right) \otimes_{\mathrm{ss}}\left(\Phi^{\prime}, \mu_{0} U^{\prime}\right)\right] .
$$

Proof. We write $(\Phi, U)=\left[0, r_{1}-1\right] \otimes\left(\Phi_{1}, S_{1}\right)$ and $\left(\Phi^{\prime}, U^{\prime}\right)=\left[0, r_{2}-1\right] \otimes\left(\Phi_{2}, S_{2}\right)$, with $\left(\Phi_{i}, S_{i}\right)$ irreducible. Let's first do the irreducible case, we are going to show that

$$
\left(\Phi_{1}, \lambda S_{1}\right) \otimes_{\mathrm{ss}}\left(\Phi_{2}, \mu S_{2}\right)=\left(\left(\Phi_{1}, \lambda S_{2}\right) \otimes\left(\Phi_{2}, \mu S_{2}\right)\right)_{\mathrm{ss}}
$$

is equivalent to

$$
\left(\Phi_{1}, S_{1}\right) \otimes_{\mathrm{ss}}\left(\Phi_{2}, S_{2}\right)=\left(\left(\Phi_{1}, S_{1}\right) \otimes\left(\Phi_{2}, S_{2}\right)\right)_{\mathrm{ss}}
$$

for $(\lambda, \mu) \in\left(\overline{\mathbb{F}_{\ell}} \times\right)^{2}$ outside a finite number of hyperplanes of $\left(\overline{\mathbb{F}_{\ell}}\right)^{2}$.

We let

$$
\operatorname{Spec}\left(S_{1}\right)-\{0\}=\left\{a_{1}, \ldots, a_{m}\right\}, \quad \operatorname{Spec}\left(S_{2}\right)-\{0\}=\left\{b_{1}, \ldots, b_{n}\right\}
$$

and put

$Z\left(S_{1}, S_{2}\right)=\left\{(\lambda, \mu) \in\left(\overline{\mathbb{F}}_{\ell} \times\right)^{2}, \forall i, j, \lambda a_{i}+\mu b_{j} \neq 0\right.$, and $\left.\forall(i, j) \neq(k, l), \lambda a_{i}+\mu b_{j} \neq \lambda a_{k}+\mu b_{l}\right\}$. 
For $(\lambda, \mu)$ in $Z\left(S_{1}, S_{2}\right)$, all $S(\lambda, \mu)=\lambda S_{1} \otimes \operatorname{Id}+\operatorname{Id} \otimes \mu S_{2}$ have the same eigenspaces in the sense that they have the same kernel, and for $(\lambda, \mu)$ and $\left(\lambda^{\prime}, \mu^{\prime}\right)$ in $Z\left(S_{1}, S_{2}\right)$, the eigenspace of $S(\lambda, \mu)$ corresponding to $\lambda a_{i}+\mu b_{j}$ is the same space as the eigenspace of $S\left(\lambda^{\prime}, \mu^{\prime}\right)$ corresponding to $\lambda^{\prime} a_{i}+\mu^{\prime} b_{j}$. In particular $S(\lambda, \mu)$ and $S\left(\lambda^{\prime}, \mu^{\prime}\right)$ are polynomials in one another. This implies that for $(\lambda, \mu)$ and $\left(\lambda^{\prime}, \mu^{\prime}\right)$ in $Z\left(S_{1}, S_{2}\right)$, if $\left(\Phi_{*}, U_{*}\right)$ is an irreducible subquotient of $\left(\left(\Phi_{1} \otimes\right.\right.$ $\Phi_{2}$ ), $S(\lambda, \mu)$ ) (see Proposition 4.36) with $U_{*}$ the Deligne endomorphism of $V_{\Phi_{*}}$ induced by $S(\lambda, \mu)$, then $\left(\Phi_{*}, T_{*}\right)$ is also an irreducible subquotient of $\left(\left(\Phi_{1} \otimes \Phi_{2}\right), S\left(\lambda^{\prime}, \mu^{\prime}\right)\right)$ with $T_{*}$ the Deligne endomorphism of $V_{\Phi_{*}}$ induced by $S\left(\lambda^{\prime}, \mu^{\prime}\right)$. As $U_{*}$ and $T_{*}$ are bijective together, or zero together, Theorem 4.24 and Lemma 4.16 imply that $\left[\Phi_{*}, U_{*}\right]=\left[\Phi_{*}, T_{*}\right]$ (both representations have the same dimension and are supported on the same irreducible line). Now let's go back to the indecomposable case.

For $(\lambda, \mu)$ and $\left(\lambda^{\prime}, \mu^{\prime}\right)$ in $Z\left(S_{1}, S_{2}\right)$ :

$$
\begin{gathered}
(\Phi, \lambda U) \otimes_{\mathrm{ss}}\left(\Phi^{\prime}, \mu U^{\prime}\right)=\left(\Phi\left(r_{1}\right), \lambda N\left(r_{1}\right)\right) \otimes\left(\Phi\left(r_{2}\right), \mu N\left(m_{2}\right)\right) \otimes\left(\Phi_{1}, \lambda S_{1}\right) \otimes_{\mathrm{ss}}\left(\Phi_{2}, \mu S_{2}\right) \\
=\left(\Phi\left(r_{1}\right), \lambda N\left(r_{1}\right)\right) \otimes\left(\Phi\left(r_{2}\right), \mu N\left(m_{2}\right)\right) \otimes\left(\Phi_{1}, \lambda S_{1}\right) \otimes_{\mathrm{ss}}\left(\Phi_{2}, \mu S_{2}\right)
\end{gathered}
$$

according to Proposition 4.9. But

$$
\left(\Phi_{1}, \lambda S_{1}\right) \otimes_{\mathrm{ss}}\left(\Phi_{2}, \mu S_{2}\right) \sim\left(\Phi_{1}, \lambda^{\prime} S_{1}\right) \otimes_{\mathrm{ss}}\left(\Phi_{2}, \mu^{\prime} S_{2}\right)
$$

and the $\mathrm{W}_{F}$-representation $\Phi\left(r_{1}\right) \otimes \Phi\left(r_{2}\right)$ is a direct sum of characters, hence this implies

$$
(\Phi, \lambda U) \otimes_{\mathrm{ss}}\left(\Phi^{\prime}, \mu U^{\prime}\right) \sim\left(\Phi\left(r_{1}\right), N\left(r_{1}\right)\right) \otimes\left(\Phi\left(r_{2}\right), N\left(r_{2}\right)\right) \otimes\left(\Phi_{1}, \lambda^{\prime} S_{2}\right) \otimes_{\mathrm{ss}}\left(\Phi_{2}, \mu^{\prime} S_{2}\right),
$$

which is itself isomorphic to

$$
\left(\Phi\left(m_{1}\right), \lambda^{\prime} N\left(m_{1}\right)\right) \otimes\left(\Phi\left(m_{2}\right), \mu^{\prime} N\left(m_{2}\right)\right) \otimes\left(\Phi_{1}, \lambda^{\prime} C_{2}\right) \otimes_{\mathrm{ss}}\left(\Phi_{2}, \mu^{\prime} S_{2}\right)=(\Phi, \lambda U) \otimes_{\mathrm{ss}}\left(\Phi^{\prime}, \mu U^{\prime}\right)
$$

thanks to Proposition 4.9 . Finally one can set $Z\left(U, U^{\prime}\right)=Z\left(S_{1}, S_{2}\right)$

For the general case, as $[\Phi, U] \oplus\left[\Phi^{\prime}, U^{\prime}\right]$ is a well defined operation from

$$
\left[\operatorname{Rep}_{\mathrm{ss}}\left(\mathrm{D}, \overline{\mathbb{F}_{\ell}}\right)\right] \times\left[\operatorname{Rep}_{\mathrm{ss}}\left(\mathrm{D}, \overline{\mathbb{F}_{\ell}}\right)\right]
$$

to $\left[\operatorname{Rep}_{\mathrm{ss}}\left(\mathrm{D}, \overline{\mathbb{F}_{\ell}}\right)\right]$, we then extend the definition of $\otimes_{\mathrm{ss}}$ by distributivity (bilinearity) from $\left[\operatorname{Indec}_{\mathrm{ss}}\left(\mathrm{D}, \overline{\mathbb{F}_{\ell}}\right)\right]$ to $\left[\operatorname{Rep}_{\mathrm{ss}}\left(\mathrm{D}, \overline{\mathbb{F}_{\ell}}\right)\right]$.

Remark 4.40. If $(\Phi, U)$ and $\left(\Phi^{\prime}, U^{\prime}\right)$ are in $\operatorname{Rep}_{\mathrm{ss}}\left(\mathrm{D}, \mathbb{F}_{l}\right)$ with $U$ or $U^{\prime}$ both bijective, and if $\left(\Phi_{0}, U_{0}\right) \in[\Phi, U] \otimes_{\mathrm{ss}}\left[\Phi^{\prime}, U^{\prime}\right]$, then $U_{0}$ is bijective. Indeed it suffices to check this when $(\Phi, U)$ and $\left(\Phi^{\prime}, U^{\prime}\right)$ are both indecomposable. It then follows from the proof of Theorem 4.39 that $S(\lambda, \mu)_{\text {ss }}$ is bijective for $(\lambda, \mu) \in Z\left(U, U^{\prime}\right)$, hence $U_{0}$ too.

\subsection{Reduction modulo $\ell$ of Deligne representations}

We now define the reduction modulo $\ell$ of an integral $\ell$-adic Deligne representation, we do this via the following decomposition which follows from Proposition 4.29 .

Definition 4.41. An element $(\Phi, U)$ of $\operatorname{Nilp}_{\mathrm{ss}}(\mathrm{D}, R)$ supported on an irreducible line $\mathbb{Z}_{\Psi}$ can be written in a unique manner as

$$
(\Phi, U)=\bigoplus_{i \geqslant 1} \bigoplus_{k \in \mathbb{Z}} a_{i, k}[0, i-1] \otimes \nu^{k} \Psi
$$

with almost all $a_{i, k} \in \mathbb{N}$ equal to zero. We call this decomposition the standard form of $(\Phi, U)$. 
We denote by $\operatorname{Rep}_{\mathrm{ss}}\left(\mathrm{D}, \overline{\mathbb{Q}_{\ell}}\right)_{e}$ the classes of $(\Phi, U) \in \operatorname{Rep}_{\mathrm{ss}}\left(\mathrm{D}, \overline{\mathbb{Q}_{\ell}}\right)_{e}$ with $\Phi$ integral, and set $\operatorname{Nilp}_{\mathrm{ss}}\left(\mathrm{D}, \overline{\mathbb{Q}_{\ell}}\right)_{\ell}=\operatorname{Nilp}_{\mathrm{ss}}\left(\mathrm{D}, \overline{\mathbb{Q}_{\ell}}\right) \cap \operatorname{Rep}_{\mathrm{ss}}\left(\mathrm{D}, \overline{\mathbb{Q}_{\ell}}\right)_{e}$.

Definition 4.42. Let $(\Phi, U) \in \operatorname{Nilp}_{\mathrm{ss}}\left(\mathrm{D}, \overline{\mathbb{Q}_{\ell}}\right)_{e}$ be supported on an irreducible line $\mathbb{Z}_{\Theta}$, with $\Theta \in$ $\operatorname{Irr}\left(\mathrm{W}_{F}, \overline{\mathbb{Q}_{\ell}}\right)$ necessarily integral, and with standard form

$$
(\Phi, U)=\bigoplus_{i \geqslant 1} \bigoplus_{k \in \mathbb{Z}} a_{i, k}[0, i-1] \otimes \nu^{k} \Theta .
$$

We define the reduction modulo $\ell$ of $(\Phi, U)$ by

$$
r_{\ell}(\Phi, U)=\bigoplus_{i \geqslant 1} \bigoplus_{k \in \mathbb{Z}} a_{i, k}[0, i-1] \otimes \nu^{k} r_{\ell}(\Theta) .
$$

We extend this definition to all of $\operatorname{Rep}_{\mathrm{ss}}\left(\mathrm{D}, \overline{\mathbb{Q}_{\ell}}\right)_{e}$ via the direct sum (as indecomposable representations are supported on irreducible lines).

To understand the structure of the reduction modulo $\ell$ of $(\Phi, U)$ above, it is useful to recall that $r_{\ell}(\Theta)$ is either irreducible, or of the form

$$
r_{\ell}(\Theta)=\mathrm{St}_{a}\left(\mathbb{Z}_{\Psi}\right)
$$

for $a \geqslant 0$ and $\Psi \in \operatorname{Irr}\left(\mathrm{W}_{F}, \overline{\mathbb{F}_{\ell}}\right)$ thanks to Proposition 3.8

\section{$5 \quad$ Local constants of Deligne representations}

In this section, we extend definitions of Artin-Deligne local constants to our setting of $\mathrm{W}_{F^{-}}$ semisimple Deligne $R$-representations with $R=\overline{\mathbb{Q}_{\ell}}$ or $\overline{\mathbb{F}_{\ell}}$. We fix $\psi: F \rightarrow \overline{\mathbb{Q}}_{\ell} \times$ a necessarily integral nontrivial character, and by abuse of notation also write $\psi$ for $r_{\ell}(\psi)$.

\subsection{Definition and basic properties}

We begin with the Artin $L$-factor:

Definition 5.1. Let $(\Phi, U) \in \operatorname{Rep}_{\mathrm{ss}}(\mathrm{D}, R)$, we set

$$
L(X,(\Phi, U))=\operatorname{det}\left(\left.(\operatorname{Id}-X \Phi(\text { Frob }))\right|_{\operatorname{Ker}(U)^{I_{F}}}\right)^{-1} .
$$

In particular, $L(X,(\Phi, U))=1$ whenever $U$ is bijective.

To define $\epsilon, \gamma$-factors of $\mathrm{W}_{F}$-semisimple Deligne $R$-representations, we first need to recall the well-known definitions of $\epsilon, \gamma$-factors of irreducible $R$-representations of $\mathrm{W}_{F}$. Let $\Psi$ be an irreducible $R$-representation of $\mathrm{W}_{F}$. The epsilon factor $\epsilon(X, \Psi, \psi)$ is defined in [8]. If $\Psi$ is not an unramified character, we set

$$
\gamma(X, \Psi, \psi)=\epsilon(X, \Psi, \psi) .
$$

If $\Psi=\chi$ is an unramified character, viewing $\chi$ as a character of $F^{\times}$via the Artin reciprocity map of local class field theory, we take $\gamma(X, \chi, \psi)$ to be the Tate $\gamma$-factor defined in Tate's thesis [18] when $R=\overline{\mathbb{Q}_{\ell}}$, and in [13, 12] when $R=\overline{\mathbb{F}_{\ell}}$.

We can now define $\epsilon, \gamma$-factors of $\mathrm{W}_{F}$-semisimple Deligne $R$-representations: 
Definition 5.2. Let $(\Phi, U) \in \operatorname{Rep}_{\mathrm{ss}}(\mathrm{D}, R)$, with $\Phi=\bigoplus_{i=1}^{r} \Psi_{i}$ the underlying direct sum of irreducible $R$-representations of $\mathrm{W}_{F}$.

(1) We define

$$
\gamma(X,(\Phi, U), \psi)=\prod_{i=1}^{r} \gamma\left(X, \Psi_{i}, \psi\right)
$$

(2) We define

$$
\epsilon(X,(\Phi, U), \psi)=\gamma(X,(\Phi, U), \psi) \frac{L(X,(\Phi, U))}{L\left(q^{-1} X^{-1},(\Phi, U)^{\vee}\right)}
$$

The following proposition follows from the definitions:

Proposition 5.3. The maps $(\Phi, U) \mapsto L(X,(\Phi, U)),(\Phi, U) \mapsto \gamma(X,(\Phi, U), \psi)$, hence the map $(\Phi, U) \mapsto \epsilon(X,(\Phi, U), \psi)$ are multiplicative with respect to direct sums.

Corollary 5.4. Two equivalent $\mathrm{W}_{F}$-semisimple Deligne $R$-representations have the same constants $L, \gamma$ and $\epsilon$, in particular we can talk of the local constants $L, \gamma$ and $\epsilon$ of $[\Phi, U] \epsilon$ $\left[\operatorname{Rep}_{\mathrm{ss}}\left(\mathrm{D}, \mathbb{F}_{l}\right)\right]$.

Proof. By Proposition 5.3 one reduces to the indecomposable case. But if $(\Phi, U)$ is indecomposable, then $L(X,(\Phi, U))$ and $L(X,(\Phi, \lambda U))$ are equal for $\lambda \neq 0$ as $\operatorname{Ker}(U)=\operatorname{Ker}(\lambda U)$, and the definition of $\gamma(X,(\Phi, U), \psi)$ and $\gamma(X,(\Phi, \lambda U), \psi)$ does not see $U$ and $\lambda U$. Finally, the result for $\epsilon$ follows.

The next proposition also follows from the definitions and the known multiplicativity properties of $\gamma$-factors in the $\ell$-adic case.

Proposition 5.5. (1) When $R=\overline{\mathbb{Q}_{\ell}}$, the factors defined in Definitions 5.1 and 5.2 are the usual $L, \gamma$ and $\epsilon$-factors from [8, 8.12], (see [10] for $\gamma$ ).

(2) When $R=\overline{\mathbb{F}_{\ell}}$, if $\Phi$ is irreducible as an $\overline{\mathbb{F}_{\ell}}$-representation of $\mathrm{W}_{F}$ and $U=0$, then

$$
L(X,(\Phi, 0))=L(X, \Phi)
$$

is the usual Artin $L$-factor of $\Phi$, and

$$
\epsilon(X,(\Phi, 0), \psi)=\epsilon(X, \Phi, \psi)
$$

is also the usual $\epsilon$-factor defined in [8].

Proof. The second assertion follows from the definitions. Suppose that $R=\overline{\mathbb{Q}_{\ell}}$, the quickest way to check our the first assertion is to use [10, Lemma 4.4]. Let $\gamma^{\prime}$ be the $\gamma$ factor defined in

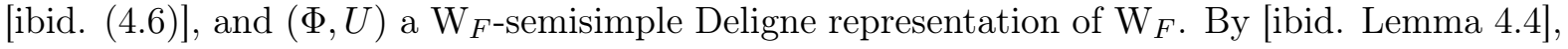
one has

$$
\gamma^{\prime}(X,(\Phi, U), \psi)=\gamma^{\prime}(X, \Phi, \psi)=\epsilon(X, \Phi, \psi) \frac{L\left(q^{-1} X^{-1}, \Phi^{\vee}\right)}{L(X, \Phi)},
$$

where $\epsilon$ and $L$ are the local constants attached to representations of $\mathrm{W}_{F}$ in [8]. In particular as they are multiplicative with respect to direct sum, this implies that $\gamma^{\prime}$ is, hence if $\Phi=\bigoplus_{i=1}^{r} \Psi_{i}$ with $\Psi_{i}$ irreducible representations of $\mathrm{W}_{F}$, one has

$$
\gamma^{\prime}(X,(\Phi, U), \psi)=\prod_{i=1}^{r} \gamma^{\prime}\left(X, \Psi_{i}, \psi\right) .
$$


So to prove that $\gamma$ is equal to $\gamma^{\prime}$, it suffices to check that $\gamma^{\prime}(X, \Psi, \psi)=\gamma(X, \Psi, \psi)$ when $\Psi$ is an irreducible representation of $\mathrm{W}_{F}$. If $\Psi$ is not an unramified character then

$$
\gamma^{\prime}(X, \Psi, \psi)=\epsilon(X, \Psi, \psi)=\gamma(X, \Psi, \psi) .
$$

If $\chi$ is an unramified character, the assertion for $L$ follows from the definitions, hence the assertion for $\epsilon$ is equivalent to $\gamma^{\prime}=\gamma$, which is by definition of $\epsilon$ in this case.

When $R=\overline{\mathbb{Q}_{\ell}}$, the constant $\epsilon(X,(\Phi, U), \Psi)$ is an invertible element of the ring $R\left[X^{ \pm 1}\right]$. Let's check this when $R=\overline{\mathbb{F}_{\ell}}$. We will write $P(X) \underset{\overline{\mathbb{F}}_{\ell}\left[X^{ \pm 1}\right]^{\times}}{\sim} Q(X)$ if $P, Q \in \overline{\mathbb{F}_{\ell}}(X)$ are equal up to an element in $\overline{\mathbb{F}_{\ell}}\left[X^{ \pm 1}\right]^{\times}$.

Proposition 5.6. Let $(\Phi, U)$ be an $\ell$-modular $\mathrm{W}_{F}$-semisimple Deligne representation, then

$$
\epsilon(X,(\Phi, U), \Psi) \in \overline{\mathbb{F}_{\ell}}\left[X^{ \pm 1}\right]^{\times} .
$$

Proof. Thanks to Proposition 5.3 and Corollary 5.4, we can suppose that $(\Phi, U)=[0, r-1] \otimes \Psi$ or $(\Phi, U)=[0, r-1] \otimes \mathcal{C}(\Psi, I)$.

Let's start with the second case. As $L(X,(\Phi, U))=1$ we have

$$
\epsilon(X,(\Phi, U), \psi)=\gamma(X,(\Phi, U), \psi)=\prod_{k=0}^{o(\Psi)-1} \gamma\left(X, \nu^{k} \Psi, \psi\right)^{r}
$$

If $\Psi$ is either ramified, or a non-banal character $\chi$ (non-banal meaning $o(\chi)=o(\nu)=1$, or equivalently $q \equiv 1[\ell])$ then $\gamma\left(X, \nu^{k} \chi, \psi\right)=\epsilon\left(X, \nu^{k} \chi, \psi\right)$ and we are done.

If $\Psi$ is a banal unramified character $\chi$ (i.e. $o=o(\nu)=o(\chi) \geqslant 2$ ) with $\chi(\varpi)=t \in \overline{\mathbb{F}}_{\ell} \times$

$$
\prod_{k=0}^{o-1} L\left(X, \nu^{k} \chi\right)=\prod_{k=0}^{o-1}\left(1-t q^{-k} X\right)^{-1}=\left(1-(t X)^{o}\right)^{-1}
$$

and similarly

$$
\prod_{k=0}^{o-1} L\left(q^{-1} X^{-1}, \nu^{-k} \chi^{-1}\right)=\left(1-(t X)^{-o}\right)^{-1}
$$

hence

$$
\epsilon(X,(\Phi, U), \psi)=\prod_{k=0}^{o-1} \epsilon\left(X, \nu^{k} \chi, \psi\right)^{r}\left(-(t X)^{o}\right)^{r} \in \overline{\mathbb{F}_{\ell}}\left[X^{ \pm 1}\right]^{\times} .
$$

It remains to deal with the case $(\Phi, U)=[0, r-1] \otimes \Psi$. If $\Psi$ is not an unramified character or if $\Psi$ is a non banal unramified character then

$$
\epsilon(X,(\Phi, U), \psi)=\gamma(X,(\Phi, U), \psi)=\prod_{i=0}^{r-1} \gamma\left(X, \nu^{i} \Phi, \psi\right)=\prod_{i=0}^{r-1} \epsilon\left(X, \nu^{i} \Phi, \psi\right) \in \overline{\mathbb{F}_{\ell}}\left[X^{ \pm 1}\right]^{\times}
$$

If $\Psi$ is a banal unramified character $\chi(q \not \equiv 1[\ell])$ with $\chi(\varpi)=t \in{\overline{\mathbb{F}_{\ell}}}^{\times}$, then

$$
\epsilon(X,(\Phi, U), \psi)=\gamma(X,(\Phi, U), \psi) \frac{L(X,(\Phi, U))}{L\left(q^{-1} X^{-1},(\Phi, U)^{\vee}\right)}=\gamma(X,(\Phi, U), \psi) \frac{\left(1-t^{-1} q^{-1} X^{-1}\right)}{\left(1-t q^{1-r} X\right)}
$$


However

$$
\gamma(X,(\Phi, U), \psi)=\prod_{i=0}^{r-1} \gamma\left(X, \nu^{i} \chi, \psi\right)=\prod_{i=0}^{r-1} \epsilon\left(X, \nu^{i} \chi, \psi\right) \prod_{i=0}^{r-1} \frac{\left(1-t q^{-i} X\right)}{\left(1-t^{-1} q^{i-1} X^{-1}\right)}
$$

Now

$$
\prod_{i=0}^{r-1} \frac{\left(1-t q^{-i} X\right)}{\left(1-t^{-1} q^{i-1} X^{-1}\right)}=\frac{\prod_{i=0}^{r-1}\left(1-t q^{-i} X\right)}{\prod_{i=-1}^{r-2}\left(1-t^{-1} q^{i} X^{-1}\right)} \underset{\overline{\mathbb{F}_{\ell}}\left[X^{ \pm 1}\right] \times}{\sim} \frac{\left(1-t q^{1-r} X\right)}{\left(1-t^{-1} q^{-1} X^{-1}\right)}
$$

hence $\epsilon(X,(\Phi, U), \psi) \underset{\overline{\mathbb{F}_{\ell}}\left[X^{ \pm 1}\right]^{\times}}{\sim} 1$.

We finally prove the multiplicativity relation for the $L$-factors of nilpotent Deligne $R$-representations:

Lemma 5.7. Let $\Psi$ and $\Psi^{\prime}$ be two irreducible $R$-representations of $\mathrm{W}_{F}$, and $n \geqslant m \geqslant 1$, then:

$$
L\left(X,([0, n-1] \otimes \Psi) \otimes_{\mathrm{ss}}\left([0, m-1] \otimes \Psi^{\prime}\right)\right)=\prod_{k=0}^{m-1} L\left(X, \nu^{n-1} \Psi \otimes_{\mathrm{ss}} \nu^{k} \Psi^{\prime}\right) .
$$

Proof. By definition

$$
([0, n-1] \otimes \Psi) \otimes_{\mathrm{ss}}\left([0, m-1] \otimes \Psi^{\prime}\right)=[0, n-1] \otimes[0, m-1] \otimes\left(\Psi \otimes_{\mathrm{ss}} \Psi^{\prime}\right) .
$$

Moreover, writing as usual $[0, r-1]=(\Phi(r), N(r))$, as $\Phi(n)^{I_{F}}=\Phi(n)$ and $\Phi(m)^{I_{F}}=\Phi(m)$, we deduce that

$$
\left(\Phi(n) \otimes \Phi(m) \otimes\left(\Psi \otimes_{\mathrm{ss}} \Psi^{\prime}\right)\right)^{I_{F}}=\Phi(n) \otimes \Phi(m) \otimes\left(\Psi \otimes_{\mathrm{ss}} \Psi^{\prime}\right)^{I_{F}} .
$$

Hence we can suppose that $\Psi$ and $\Psi^{\prime}$ are both the trivial character to prove the multiplicativity relation. Writing $\Phi(l)=\bigoplus_{i=0}^{l-1} \nu^{i} . e_{i}$, one can decompose $\Phi(n) \otimes \Phi(m)$ as $\bigoplus_{j=0}^{n+m-2} V_{k}$, where $V_{k}=$ $\operatorname{Vect}\left(e_{i} \otimes e_{j}\right)_{i+j=k}$. Write $N=N(n) \oplus \operatorname{Id}+\operatorname{Id} \otimes N(m)$, then $N\left(V_{k}\right) \subseteq V_{k+1}$ for $k<n+m-2$ and $N\left(V_{n+m-2}\right)=\{0\}$. Hence

$$
\operatorname{Ker}(N)=\bigoplus_{j=0}^{n+m-2} \operatorname{Ker}(N) \cap V_{k}
$$

However one checks that $\operatorname{Ker}(N) \cap V_{k}=\{0\}$ for $j<n-1$, and

$$
\operatorname{Ker}(N) \cap V_{n-1+k}=\operatorname{Vect}\left(\sum_{i=0}^{m-1}(-1)^{i} e_{n+m-2-i} \otimes e_{i}\right)
$$

for $k=0, \ldots, m-1$. Denoting $K_{k}$ the space $\operatorname{Ker}(N) \cap V_{k}$, we have showed that

$$
\operatorname{Ker}(N)=\bigoplus_{k=0}^{m-1} K_{n-1+k}
$$

where $\mathrm{W}_{F}$ acts via $\nu^{n-1+k}$ on the line $K_{n-1+k}$, and the result follows.

\subsection{Reduction modulo $\ell$ of local constants}

We state two results concerning good reduction of local constants. The first is a result of [10], which states that $\gamma$-factors are compatible with reduction modulo $\ell$. For $P \in \overline{\mathbb{Z}_{\ell}}[X]$, we denote by $r_{\ell}(P)=\overline{\mathbb{F}_{\ell}}[X]$ the polynomial obtained by applying $r_{\ell}$ to the coefficients of $P$. For $Q$ in $\overline{\mathbb{Z}_{\ell}}[X]$, such that $r_{\ell}(Q) \neq 0$, we set $r_{\ell}(P / Q)=r_{\ell}(P) / r_{\ell}(Q)$. 
Proposition 5.8. [10, Theorem 1.1, 1]: Let $\Phi$ be an integral semisimple representation of $\mathrm{W}_{F}$, then

$$
r_{\ell}(\gamma(X, \Phi, \psi))=\gamma\left(X, r_{\ell}(\Phi), \psi\right)
$$

Then we state the following immediate consequence of Proposition 3.13 .

Theorem 5.9. Let $\Psi$ and $\Psi^{\prime}$ be banal irreducible $\overline{\mathbb{F}_{\ell}}$-representations of $\mathrm{W}_{F}$, and let $\tilde{\Psi}$ and $\tilde{\Psi}^{\prime}$ be irreducible $\ell$-adic lifts of $\Psi$ and $\Psi^{\prime}$ respectively, then

$$
L\left(X, \Psi \otimes_{\mathrm{ss}} \Psi^{\prime}\right)=r_{\ell}\left(L\left(X, \tilde{\Psi} \otimes \tilde{\Psi}^{\prime}\right)\right) .
$$

Proof. Because $\tilde{\Psi} \otimes \tilde{\Psi}^{\prime}$ is integral,

$$
\operatorname{det}\left(\left.(\mathrm{Id}-X \Phi(\text { Frob }))\right|_{\left(V_{\Psi} \otimes V_{\Psi^{\prime}}\right)^{I_{F}}}\right) \in \overline{\mathbb{Z}_{\ell}}[X],
$$

hence it makes sense to consider $r_{\ell}\left(L\left(X, \tilde{\Psi} \otimes \tilde{\Psi}^{\prime}\right)\right)$.

Moreover thanks to Proposition 3.13 , we know that $\overline{\left(V_{\tilde{\Psi}} \otimes V_{\tilde{\Psi}^{\prime}}\right)^{I_{F}}}=\left(V_{\Psi} \otimes V_{\Psi^{\prime}}\right)^{I_{F}}$, hence

$$
r_{\ell}\left(L\left(X, \tilde{\Psi} \otimes \tilde{\Psi}^{\prime}\right)\right)=\operatorname{det}\left(\left.\left(\operatorname{Id}-X \Psi \otimes \Psi^{\prime}(\text { Frob })\right)\right|_{\left(V_{\Psi} \otimes V_{\Psi^{\prime}}\right)^{I_{F}}}\right)^{-1} .
$$

Finally according to [ibid.] again, we have $\left(\left(V_{\Psi} \otimes V_{\Psi^{\prime}}\right)^{I_{F}}\right)_{\mathrm{Ss}} \simeq\left(V_{\Psi} \otimes_{\mathrm{SS}} V_{\Psi^{\prime}}\right)^{I_{F}}$, hence

$$
\operatorname{det}\left(\left.(\mathrm{Id}-X \Phi(\text { Frob }))\right|_{\left(V_{\Psi} \otimes V_{\Psi^{\prime}}\right)^{I} F}\right)^{-1}=L\left(X, \Psi \otimes_{\mathrm{SS}} \Psi^{\prime}\right) .
$$

\section{The $\ell$-modular Langlands correspondence and local constants}

In [24], Vignéras defines a bijection $\mathrm{V}$ (Theorem 6.3) between $\coprod_{n \geqslant 0} \operatorname{Irr}\left(G_{n}, \overline{\mathbb{F}_{\ell}}\right)$ (see Notation 6.1) and $\operatorname{Nilp}_{\mathrm{ss}}\left(\mathrm{D}, \overline{\mathbb{F}_{\ell}}\right)$, the "semisimplification" of which is obtained by "reducing modulo $\ell$ " the $\ell$-adic LLC, and which moreover commutes with character twists, taking duals, and takes central characters to determinant (Lemma 6.4). The aim of this last section is to define an injection $\mathrm{C}$ (Definition 6.3) of $\operatorname{Irr}\left(G, \overline{\mathbb{F}_{\ell}}\right)$ into $\left[\operatorname{Rep}_{\mathrm{ss}}\left(\mathrm{D}, \overline{\mathbb{F}_{\ell}}\right)\right]$, which besides sharing all these properties with $\mathrm{V}$, takes the local factors of pairs of generic representations defined in [12] to those of tensor products of elements in $\left[\operatorname{Rep}_{\mathrm{D}, \mathrm{ss}}\right]$ (Theorem 6.13).

\subsection{Representations of $\mathrm{GL}_{n}(F)$}

We put $G_{n}=\mathrm{GL}_{n}(F)$ ( $G_{0}$ is trivial by convention), and denote by $N_{n}$ the group of unipotent upper triangular matrices in $G_{n}$. For $n \in N_{n}$ and $\psi: F \rightarrow R^{\times}$a non-trivial character, we set

$$
\theta_{\psi}(n)=\psi\left(\sum_{i=1}^{n-1} n_{i, i+1}\right) .
$$

We say that an irreducible representation $\pi$ of $G_{n}$ is generic if

$$
\operatorname{Hom}_{N_{n}}\left(\pi, \theta_{\psi}\right) \neq\{0\},
$$

in which case it is well known that $\operatorname{dim}_{R}\left(\operatorname{Hom}_{N_{n}}\left(\pi, \theta_{\psi}\right)\right)=1$, but we do not use this multiplicity one property. 
For $\left\{n_{1}, \ldots, n_{r}\right\}$ positive integers, let $\pi_{i} \in \operatorname{Rep}\left(G_{n_{i}}, R\right)$ and put $n=\sum_{i=1}^{r} n_{i}$. We denote by

$$
\pi_{1} \times \cdots \times \pi_{r} \in \operatorname{Rep}\left(G_{n}, R\right)
$$

the normalized parabolic induction of the $\pi_{i}$ 's. An irreducible $R$-representation of $G_{n}$ is called cuspidal if it does not appear as a subrepresentation of a properly parabolically induced representation. It is called supercuspidal if it does not appear as a subquotient of a properly parabolically induced representation. By classical results (see for example [2], 27] and [22]), if the $\pi_{i}$ 's are generic, then $\pi_{1} \times \cdots \times \pi_{r}$ has a unique generic subquotient and cuspidal representations are always generic. If $\rho$ is cuspidal, we denote by $\operatorname{St}(r, \rho)$ the unique generic subquotient of $\rho \times \nu \rho \times \cdots \times \nu^{r-1} \rho$. By convention, $\operatorname{St}(0, \rho)$ is the trivial representation of the trivial group $G_{0}$.

On the other hand, in [2] when $R=\overline{\mathbb{Q}_{\ell}}$, and [22] or [14, Definitions 7.5] when $R=\overline{\mathbb{F}_{\ell}}$, to a cuspidal segment

$$
[a, b]_{\rho}=\left(\nu^{a} \rho, \ldots, \nu^{b} \rho\right)
$$

with $a \leqslant b$, the authors attach a certain irreducible quotient $\mathrm{L}\left([a, b]_{\rho}\right)$ and a certain irreducible submodule $\mathrm{Z}\left([a, b]_{\rho}\right)$ of

$$
\nu^{a} \rho \times \cdots \times \nu^{b} \rho,
$$

which are respectively the cosocle and socle of it when $R=\overline{\mathbb{Q} \ell}$. The length of the segment $[a, b]_{\rho}$ is $b-a+1$. By convention if $b<a$, then $[a, b]_{\rho}$ is the empty segment and $\mathrm{L}\left([a, b]_{\rho}\right)$ and $\mathrm{Z}\left([a, b]_{\rho}\right)$ are the trivial representation of $G_{0}$.

Consider two cuspidal segments $\Delta$ and $\Delta^{\prime}$, we say that $\Delta$ precedes $\Delta^{\prime}$ if one can extract a segment longer than both from the sequence $\left(\Delta, \Delta^{\prime}\right)$, in which case we set $\Delta \prec \Delta^{\prime}$. We say that two cuspidal segments are linked if either of them precedes the other one, otherwise we say that they are unlinked.

We denote by * the Aubert-Zelevinsky involution on $\operatorname{Irr}\left(G_{n}, R\right)$ for any $n \geqslant 0$ (see [27, 1] when $R=\overline{\mathbb{Q}_{\ell}}$ and [21, 15] when $R=\overline{\mathbb{F}_{\ell}}$ ), it satisfies

$$
\left(\pi_{1} \times \pi_{2}\right)^{*}=\pi_{1}^{*} \times \pi_{2}^{*}
$$

when $\pi_{1} \times \pi_{2}$ is irreducible. This * involution also commutes with taking duals: in the $\ell$-modular case, using the notation of [15, Theorem 8], this property follows from the theorem itself and the fact that $\mathbf{D}$ commutes with taking duals. It is shown in these references (for example [15, Proposition 4.10]) that for a cuspidal segment $\Delta$ :

$$
\mathrm{L}(\Delta)=\mathrm{Z}(\Delta)^{*} .
$$

Notation 6.1. Let now us fix some more notation:

- $\operatorname{Irr}(G, R)=\coprod_{n \geqslant 0} \operatorname{Irr}\left(G_{n}, R\right)$.

- $\operatorname{Irr}_{\text {gen }}\left(G_{n}, R\right)$ : the set of isomorphism classes of generic representations $\operatorname{in} \operatorname{Irr}\left(G_{n}, R\right)$.

- $\operatorname{Irr}_{\text {gen }}(G, R)=\coprod_{n \geqslant 0} \operatorname{Irr}_{\text {gen }}\left(G_{n}, R\right)$.

- $\operatorname{Irr}_{\mathrm{c}}\left(G_{n}, R\right)$ : the set of isomorphism classes of cuspidal representations in $\operatorname{Irr}\left(G_{n}, R\right)$.

- $\operatorname{Irr}_{\mathrm{sc}}\left(G_{n}, R\right)$ : the set of isomorphism classes of supercuspidal representations in $\operatorname{Irr}\left(G_{n}, R\right)$.

- $\operatorname{Irr}_{\mathrm{c}}(G, R)=\coprod_{n \geqslant 0} \operatorname{Irr}_{\mathrm{c}}\left(G_{n}, R\right)$.

- $\operatorname{Irr}_{\mathrm{sc}}(G, R)=\coprod_{n \geqslant 0} \operatorname{Irr}_{\mathrm{sc}}\left(G_{n}, R\right)$. 
If $\pi \in \operatorname{Rep}(G, R)$ has a central character (for example if it belongs to $\operatorname{Irr}(G, R$ ), we denote it by $c_{\pi}$. If $\rho$ is a cuspidal representation, we denote by $\mathbb{Z}_{\rho}$ the associated cuspidal line

$$
\mathbb{Z}_{\rho}=\left\{\nu^{k} \rho, k \in \mathbb{Z}\right\} .
$$

If $\rho$ is supercuspidal, we say that $\pi \in \operatorname{Irr}(G, R)$ is supported on $\mathbb{Z}_{\rho}$ if all supercuspidal representations of its supercuspidal support (which is unique by [22] or [14]) belong to $\mathbb{Z}_{\rho}$. The set $\mathbb{Z}_{\rho}$ is finite if and only if $R=\overline{\mathbb{F}_{\ell}}$, in which case we set

$$
o(\rho)=\left|\mathbb{Z}_{\rho}\right| .
$$

Following [14, Remarque 8.15], we say that $\pi \in \operatorname{Irr}(G, R)$ is banal if the cuspidal support of $\pi$ contains no cuspidal line, in particular non-banal irreducible representations exist only when $R=\overline{\mathbb{F}_{\ell}}$ and a cuspidal representation $\rho$ is non-banal if and only if $o(\rho)=1$. By [19], 22] or [14, Theorem 6.4], if a cuspidal representation $\rho$ is banal, then it is supercuspidal.

If $\tau$ is cuspidal, then there is a non-negative integer $k$ such that

$$
\tau=\operatorname{St}\left(o(\rho) \ell^{k}, \rho\right)
$$

for a supercuspidal representation $\rho$, the cuspidal line of which is unique ( $\rho$ can be replaced by any supercuspidal representation on the same line). Therefore, in this case, we set

$$
\mathrm{St}_{k}\left(\mathbb{Z}_{\rho}\right)=\operatorname{St}\left(o(\rho) \ell^{k}, \rho\right) .
$$

If $\rho$ is cuspidal, then $\mathrm{L}\left([0, r-1]_{\rho}\right)=\operatorname{St}(r, \rho)$ if and only if either $r<o(\rho)$ when $\rho$ is banal, or $r<\ell$ when $\rho$ is non-banal ([14, Remarque 8.14].

By [27, Theorem 9.7] when $R=\overline{\mathbb{Q}_{\ell}}$, and [22, Theorem V.7] or [14, Theorem 9.10] when $R=\overline{\mathbb{F}_{\ell}}$, a representation $\pi \in \operatorname{Irr}_{\text {gen }}(G)$ can be written under the form of a commutative product

$$
\pi=\mathrm{L}\left(\left[0, m_{1}-1\right]_{\rho_{1}}\right) \times \cdots \times \mathrm{L}\left(\left[0, m_{r}-1\right]_{\rho_{r}}\right)=\operatorname{St}\left(m_{1}, \rho_{1}\right) \times \cdots \times \operatorname{St}\left(m_{r}, \rho_{r}\right)
$$

where, for $i \in\{1, \ldots, r\}$, the cuspidal segments $\left[0, m_{i}-1\right]_{\rho_{i}}$ are unlinked and unique up to ordering.

In particular, a representation $\pi \in \operatorname{Irr}_{\text {gen }}(G, R)$ supported on a supercuspidal line $\mathbb{Z}_{\rho}, \pi$ can be written in a unique manner $\pi_{\mathrm{b}} \times \pi_{\mathrm{tnb}}$ as in [12, Proposition 2.3]. The representation $\pi_{\mathrm{b}}$ is a banal representation, which can be written in a unique manner as a (possibly empty) product

$$
\pi_{\mathrm{b}}=\prod_{i=1}^{s} \mathrm{~L}\left(\left[c_{i}, d_{i}\right]_{\rho}\right)=\prod_{i=1}^{s} \operatorname{St}\left(d_{i}-c_{i}+1, \nu^{c_{i}} \rho\right)
$$

with the segments $\left[c_{i}, d_{i}\right]_{\rho}$ unlinked (in particular all of the lengths $d_{i}-c_{i}+1$ are $<o(\rho)$, hence the product is empty if $\rho$ is non banal). The representation $\pi_{\text {tnb }}$ is totally non-banal, i.e. no segment occurring in $\pi_{\text {tnb }}$ is banal, it is a (possibly empty) product of the form

$$
\pi_{\mathrm{tnb}}=\prod_{k=0}^{r} \mathrm{~L}\left(\left[0, a_{k}-1\right]_{\mathrm{St}_{k}\left(\mathbb{Z}_{\rho}\right)}\right), \text { for } 0 \leqslant a_{k}<\ell .
$$

\subsection{The V-correspondence}

As in the context of representations of $\mathrm{W}_{F}$, there is also a notion of reduction modulo $\ell$ for integral $\ell$-adic representations of $G_{n}$. If $\pi$ is a finite length representation in $\operatorname{Rep}\left(G_{n}, \overline{\mathbb{Q}_{\ell}}\right)$, one 
says that it is integral if it contains a stable $G_{n}$-lattice $\Lambda$. We denote by a right index $e$ the set of classes of integral representations inside a set of classes of $\ell$-adic representations, for example $\operatorname{Irr}\left(G_{n}, \overline{\mathbb{Q}_{\ell}}\right)_{e}$. If $\pi$ has finite length, the $\ell$-modular representation $\pi \otimes_{\mathbb{F}_{\ell}} \Lambda$ is of finite length: we denote by $r_{\ell}(\pi)$ its semisimplification which is independent of $\Lambda$, as proved in [26, Theorem 1]. By [19, II.4.12], $\operatorname{Irr}_{c}\left(G, \overline{\mathbb{Q}_{\ell}}\right)_{e}$ are the elements $\operatorname{in}_{\operatorname{Irr}_{c}}\left(G, \overline{\mathbb{Q}_{\ell}}\right)$ with integral central character. By [19, III.5.10], if $\rho \in \operatorname{Irr}_{\mathrm{c}}\left(G_{n}, \overline{\mathbb{F}_{\ell}}\right)$, there is $\tilde{\rho} \in \operatorname{Irr}_{\mathrm{c}}\left(G_{n}, \overline{\mathbb{Q}_{\ell}}\right)_{e}$ such that $r_{\ell}(\tilde{\rho})=\rho$.

In [24, I.8.4], Vignéras introduces a surjection

$$
J_{\ell}: \operatorname{Irr}\left(G_{n}, \overline{\mathbb{Q}_{\ell}}\right)_{e} \rightarrow \operatorname{Irr}\left(G_{n}, \overline{\mathbb{F}_{\ell}}\right) .
$$

Let $\psi$ be a (necessarily integral) character of $F$ such that $r_{\ell}(\psi)$ is nontrivial. For $A$ a finite subset of $\{1, \ldots, n-1\}$, we denote by $\theta_{\psi, A}$ the (degenerate when $A \neq \emptyset$ ) character of $N_{n}$ defined by

$$
\theta_{\psi, A}(n)=\psi\left(\sum_{i \notin A} n_{i, i+1}\right)
$$

Then by [27, Theorem 8.2], for $\pi \in \operatorname{Irr}\left(G_{n}, \overline{\mathbb{Q}_{\ell}}\right)_{e}$, there is a unique $A$ minimal for the inclusion relation, such that $\pi$ has a Whittaker model (which is unique) with respect to $\theta_{\psi_{A}}$ (we will say of type A). By [14, Proposition 9.19], the reduction modulo $\ell$ of $\pi$ has a unique irreducible summand $\pi^{\prime}$ which has a Whittaker model with respect to $r_{\ell}\left(\theta_{\psi, A}\right)$. The map $J_{\ell}$ is then defined by $J_{\ell}(\pi)=\pi^{\prime}$. Let's compute some examples:

Example 6.2. (1) If $\Delta=[a, b]_{\tau}$ is a cuspidal $\ell$-adic segment so that $\rho=r_{\ell}(\tau)$ is cuspidal (see [23, Section 1] for this fact), and we set $r_{\ell}(\Delta)=[a, b]_{\rho}$, then

$$
J_{\ell}(\mathrm{Z}(\Delta))=\mathrm{Z}\left(r_{\ell}(\Delta)\right)
$$

(2) Take $\tau \in \operatorname{Irr}_{\mathrm{c}}\left(G, \mathbb{Q}_{l}\right)_{e}$ such that $\rho_{0}=r_{\ell}(\tau)$ is cuspidal non-supercuspidal. Then $\rho_{0}=\operatorname{St}_{r}\left(\mathbb{Z}_{\rho}\right)$. We set $\rho_{i}=\mathrm{St}_{r+i}\left(\mathbb{Z}_{\rho}\right)$. For $k \in \mathbb{N}$, with $\ell$-adic expansion $k=a_{0}+a_{1} \ell+\cdots+a_{d} \ell^{d}$, we have

$$
J_{\ell}(\operatorname{St}(k, \tau))=\operatorname{St}\left(a_{0}, \rho_{0}\right) \times \operatorname{St}\left(a_{1}, \rho_{1}\right) \times \cdots \times \operatorname{St}\left(a_{d}, \rho_{d}\right) .
$$

(3) Take $\tau \in \operatorname{Irr}_{\mathrm{c}}\left(G, \overline{\mathbb{Q}_{\ell}}\right)_{e}$ such that $\rho=r_{\ell}(\tau)$ is supercuspidal. For $k \in \mathbb{N}$, we write the euclidean division of $k$ by $o(\rho)$ as $k=u o(\rho)+r$. Again, we set $\rho_{i}=\operatorname{St}_{i}\left(\mathbb{Z}_{\rho}\right)$ and we write the $\ell$-adic expansion of $u$ as $u=a_{0}+a_{1} \ell+\cdots+a_{d} \ell^{d}$. Then

$$
J_{\ell}(\operatorname{St}(k, \tau))=\operatorname{St}(r, \rho) \times \operatorname{St}\left(a_{0}, \rho_{0}\right) \times \operatorname{St}\left(a_{1}, \rho_{1}\right) \times \cdots \times \operatorname{St}\left(a_{d}, \rho_{d}\right) .
$$

We denote by

$$
\text { LLC }: \operatorname{Nilp}_{\mathrm{ss}}\left(\mathrm{D}, \overline{\mathbb{Q}_{\ell}}\right) \rightarrow \operatorname{Irr}\left(G, \overline{\mathbb{Q}_{\ell}}\right),
$$

the $\ell$-adic local Langlands correspondence, characterized by compatibility with the formation of local constants of pairs, this correspondence depends on our fixed choice of square root of $q$. We now recall the main result of [24], the $\ell$-modular local Langlands correspondence, defined by compatibility with LLC and reduction modulo $\ell$ of Deligne representations (as we defined in Section 4.5), but in a non-naive way:

Theorem 6.3 ([24, Theorems 1.6 and 1.8.5]). There is a bijection

$$
\mathrm{V}: \operatorname{Irr}\left(G, \overline{\mathbb{F}_{\ell}}\right) \stackrel{\sim}{\rightarrow} \operatorname{Nilp}_{\mathrm{ss}}\left(\mathrm{D}, \overline{\mathbb{F}_{\ell}}\right),
$$

characterized by the property $\mathrm{V}\left(J_{\ell}\left(\operatorname{LLC}(\Phi)^{*}\right)^{*}\right)=r_{\ell}(\Phi)$ for any $\Phi \in \operatorname{Nilp}_{\mathrm{ss}}\left(\mathrm{D}, \overline{\mathbb{Q}_{\ell}}\right)_{e}$. Its restriction to $\operatorname{Irr}_{\mathrm{sc}}\left(G, \overline{\mathbb{F}_{\ell}}\right)$ induces a bijection between $\operatorname{Irr}_{\mathrm{sc}}\left(G, \overline{\mathbb{F}_{\ell}}\right)$ and $\operatorname{Irr}\left(\mathrm{W}_{F}, \overline{\mathbb{F}_{\ell}}\right)$. 
The following immediate properties of $\mathrm{V}$ are clear, though not explicitly stated in [24]:

Lemma 6.4. Let $\pi \in \operatorname{Irr}\left(G, \overline{\mathbb{F}_{\ell}}\right)$.

(1) For all characters $\chi: \mathrm{GL}_{n}(F) \rightarrow{\overline{\mathbb{F}_{\ell}}}^{\times}$,

$$
\mathrm{V}(\chi \pi)=\chi \mathrm{V}(\pi)
$$

(2) Letting $c_{\pi}$ denote the central character of $\pi$, then using local class field theory

$$
c_{\pi}=\operatorname{det}(\mathrm{V}(\pi))
$$

(3) We have

$$
\mathrm{V}\left(\pi^{\vee}\right)=\mathrm{V}(\pi)^{\vee}
$$

(4) For $\pi^{\prime} \in \operatorname{Irr}\left(G, \overline{\mathbb{F}_{\ell}}\right)$ such that $\pi \times \pi^{\prime}$ is irreducible, we have $\mathrm{V}\left(\pi \times \pi^{\prime}\right)=\mathrm{V}(\pi) \oplus \mathrm{V}\left(\pi^{\prime}\right)$.

(5) Moreover if $\pi=\pi\left(\mathbb{Z}_{\rho_{i}}\right) \times \cdots \times \pi\left(\mathbb{Z}_{\rho_{r}}\right)$ with $\pi\left(\mathbb{Z}_{\rho_{i}}\right)$ supported on the supercuspidal line $\mathbb{Z}_{\rho_{i}}$ and $\mathbb{Z}_{\rho_{i}} \neq \mathbb{Z}_{\rho_{j}}$ for $i \neq j$, then

$$
\mathrm{V}(\pi)=\bigoplus_{i=1}^{r} \mathrm{~V}\left(\pi\left(\mathbb{Z}_{\rho_{i}}\right)\right) .
$$

Proof. Both $\ell$-modular and $\ell$-adic Aubert-Zelevinsky involutions, $J_{\ell}$ and LLC commute with character twists, and

$$
r_{\ell}: \operatorname{Nilp}_{\mathrm{ss}}\left(\mathrm{D}, \overline{\mathbb{Q}_{\ell}}\right)_{e} \rightarrow \operatorname{Nilp}_{\mathrm{ss}}\left(\mathrm{D}, \overline{\mathbb{F}_{\ell}}\right),
$$

as well, hence the first statement. For $\pi \in \operatorname{Irr}\left(G, \overline{\mathbb{Q}_{\ell}}\right)_{e}$, the central character $c_{J_{\ell}(\pi)}$ of $J_{\ell}(\pi)$ is equal to $r_{\ell}\left(c_{\pi}\right)$, both Zelevinsky involutions do not touch the central character, and LLC takes determinant to central character. The commutation with taking duals also follows from the fact that $r_{\ell}$, both Aubert-Zelevinsky involutions, LLC and $J_{\ell}$ share this property. The next property is a consequence of the analogous property for LLC, the fact that both *-involutions commute with irreducible parabolic induction, and the fact that $J_{\ell}\left(\pi_{1} \times \pi_{2}\right)=J_{\ell}\left(\pi_{1}\right) \times J_{\ell}\left(\pi_{2}\right)$ when $\pi_{1} \times \pi_{2}$ is irreducible (this last assertion follows from standard properties of degenerate Whittaker models with respect to parabolic induction). The last property follows from the previous property.

Following Theorem 6.3, we call an element $(\Phi, U)$ of $\operatorname{Nilp}_{\mathrm{ss}}\left(\mathrm{D}, \overline{\mathbb{F}_{\ell}}\right)$ a $\mathrm{V}$-parameter and if $\mathrm{V}(\pi)=$ $(\Phi, U)$ for $\pi \in \operatorname{Irr}\left(G, \overrightarrow{\mathbb{F}}_{\ell}\right)$ we say that $(\Phi, U)$ is the $\mathrm{V}$-parameter of $\pi$. We end this section with some examples of $\mathrm{V}$-parameters.

Example 6.5. (1) If $\rho=\operatorname{St}_{k}\left(\mathbb{Z}_{\rho_{0}}\right)$ is cuspidal non supercuspidal, with $\rho_{0} \in \operatorname{Irr}_{\mathrm{sc}}(G)$ and set

$$
\Psi_{0}=\mathrm{V}\left(\rho_{0}\right) \in \operatorname{Irr}\left(\mathrm{W}_{F}, \overline{\mathbb{F}_{\ell}}\right)
$$

so that $o\left(\rho_{0}\right)=o\left(\Psi_{0}\right)$. Then if $\tilde{\rho}_{0}$ is a (necessary cuspidal) lift of $\rho_{0}$, and $\tilde{\Psi}_{0}$ is a (necessary irreducible) lift of $\Psi_{0}$, by Theorem 6.3, we have $r_{\ell}\left(\tilde{\Psi}_{0}\right)=\Psi_{0}$. Now we also have

$$
\rho=\mathrm{J}_{\ell}\left(\operatorname{St}\left(o\left(\rho_{0}\right) \ell^{k}, \tilde{\rho_{0}}\right)\right) \text {, }
$$

hence

$$
\rho=\rho^{*}=\mathrm{J}_{\ell}\left(\operatorname{St}\left(o\left(\rho_{0}\right) \ell^{k}, \tilde{\rho_{0}}\right)\right)^{*}=\mathrm{J}_{\ell}\left(\mathrm{Z}\left(\left[0, o\left(\rho_{0}\right) \ell^{k}-1\right] \tilde{\rho}_{0}\right)^{*}\right)^{*} .
$$

On the other hand

$$
r_{\ell}\left(\operatorname{LLC}\left(\mathrm{Z}\left(\left[0, o\left(\rho_{0}\right) \ell^{k}-1\right]_{\tilde{\rho}_{0}}\right)\right)=r_{\ell}\left(\bigoplus_{i=0}^{o\left(\Psi_{0}\right) \ell^{k}-1} \nu^{i} \tilde{\Psi}_{0}\right)=\ell^{k}\left(\bigoplus_{i=0}^{o\left(\Psi_{0}\right)-1} \nu^{i} \Psi_{0}\right),\right.
$$

hence

$$
\mathrm{V}\left(\mathrm{St}_{k}\left(\mathbb{Z}_{\rho_{0}}\right)\right)=\operatorname{St}_{k}\left(\mathbb{Z}_{\Psi_{0}}\right)
$$


(2) If $\rho$ is supercuspidal, and $\Psi=\mathrm{V}(\rho) \in \operatorname{Irr}\left(\mathrm{W}_{F}, \overline{\mathbb{F}_{\ell}}\right)$, then

$$
\mathrm{V}\left(\mathrm{L}\left([a, b]_{\rho}\right)\right)=[a, b] \otimes \Psi .
$$

Indeed start with an $\ell$-adic lift $\tilde{\rho}$ of $\rho$ with Langlands parameter $\tilde{\Psi}$ so that $\Psi=r_{\ell}(\tilde{\Psi})$. Then

$$
J_{\ell}\left(\mathrm{L}\left([a, b]_{\tilde{\rho}}\right)^{*}\right)^{*}=J_{\ell}\left(\mathrm{Z}\left([a, b]_{\tilde{\rho}}\right)\right)^{*}=\mathrm{Z}\left([a, b]_{\rho}\right)^{*}=\mathrm{L}\left([a, b]_{\rho}\right)
$$

but on the other hand

$$
r_{\ell}\left(\mathrm{L}\left([a, b]_{\tilde{\rho}}\right)=r_{\ell}([a, b] \otimes \tilde{\Psi})=[a, b] \otimes \Psi .\right.
$$

Similarly if $\rho=\operatorname{St}_{k}\left(\rho_{0}\right)$ is cuspidal non supercuspidal with $\mathrm{V}\left(\rho_{0}\right)=\Psi_{0}$, we find

$$
\mathrm{V}\left(\mathrm{L}\left([a, b]_{\rho}\right)\right)=[a, b] \otimes \mathrm{St}_{k}\left(\mathbb{Z}_{\Psi_{0}}\right) .
$$

(3) Take $\pi \in \operatorname{Irr}_{\text {gen }}\left(G, \overline{\mathbb{F}_{\ell}}\right)$, supported on the supercuspidal line $\mathbb{Z}_{\rho}$, and that $\mathrm{V}(\rho)=\Psi$. We write $\pi=\pi_{\mathrm{b}} \times \pi_{\mathrm{tnb}}$ where $\pi_{b}$ is banal and $\pi_{\mathrm{tnb}}$ is totally non-banal. Write

$$
\pi_{\mathrm{b}}=\prod_{i \geqslant 1} \prod_{k=0}^{o(\Psi)-1} \mathrm{~L}\left([0, i-1]_{\nu^{k} \rho}\right)^{c_{i, k}}
$$

where the occurring segments are unlinked, hence in particular for each fixed $i$, there is a $k$ such that $c_{i, k}=0$. Write

$$
\pi_{\mathrm{tnb}}=\prod_{k \geqslant 0} \mathrm{~L}\left(\left[0, a_{k}-1\right]_{\mathrm{St}_{k}(\rho)}\right)
$$

with $0 \leqslant a_{k}<\ell$. Then using a generic standard lift of $\pi$ as in [12, Definition 2.24], one checks that $\mathrm{V}(\pi)=\mathrm{V}\left(\pi_{\mathrm{b}}\right) \oplus \mathrm{V}\left(\pi_{\mathrm{tnb}}\right)$, with

$$
\begin{gathered}
\mathrm{V}\left(\pi_{\mathrm{b}}\right)=\bigoplus_{i \geqslant 1} \bigoplus_{k=0}^{o(\Psi)-1} c_{i, k}[0, i-1] \otimes \nu^{k} \Psi \\
\mathrm{V}\left(\pi_{\mathrm{tnb}}\right)=\bigoplus_{j \geqslant 0}\left[0, a_{j}-1\right] \otimes \mathrm{St}_{j}\left(\mathbb{Z}_{\Psi}\right) .
\end{gathered}
$$

\subsection{The C-correspondence}

Let $(\Phi, U)$ be a V-parameter, supported on an irreducible line $\mathbb{Z}_{\Psi}$, with standard form

$$
(\Phi, U)=\bigoplus_{i \geqslant 0} \bigoplus_{k=0}^{o(\Psi)-1} a_{i, k}[0, i-1] \otimes \nu^{k} \Psi .
$$

Definition 6.6. We say that $(\Phi, U)$ is acyclic if for each fixed $i$, there is $0 \leqslant k \leqslant o(\Psi)-1$ such that $a_{i, k}=0$. We say that $(\Phi, U)$ is cyclic if for each fixed $i$, the coefficient $a_{i, k}$ is independent of $k$.

Take a general V-parameter $(\Phi, U)$ supported on an irreducible line $\mathbb{Z}_{\Psi}$ with standard form written as above, and set

$$
b_{i}=\min _{k} a_{i, k} \text {, and } c_{i, k}=a_{i, k}-b_{i} .
$$

It can then be rewritten as

$$
(\Phi, U)=(\Phi, U)_{\mathrm{acyc}} \oplus(\Phi, U)_{\mathrm{cyc}}
$$


with

$$
\begin{aligned}
(\Phi, U)_{\mathrm{acyc}} & =\bigoplus_{i \geqslant 1} \bigoplus_{k=0}^{o(\Psi)-1} c_{i, k}[0, i-1] \otimes \nu^{k} \Psi \\
(\Phi, U)_{\mathrm{cyc}} & =\bigoplus_{j \geqslant 1} b_{j}[0, j-1] \otimes \bigoplus_{k=0}^{o(\Psi)-1} \nu^{k} \Psi .
\end{aligned}
$$

Notice that for each $i$, we have $c_{i, k}=0$ for at least one $k$ so it makes sense to call $(\Phi, U)_{\text {acyc }}$ the acyclic part of $\Phi$, and $(\Phi, U)_{\text {cyc }}$ is cyclic and we call it the cyclic part of $\Phi$. Conversely, if a $\mathrm{V}$-parameter supported on $\mathbb{Z}_{\Psi}$ is written as the sum of an acyclic and a cyclic parameter,

$$
\left(\bigoplus_{i \geqslant 1} \bigoplus_{k=0}^{o(\Psi)-1} c_{i, k}[0, i-1] \otimes \nu^{k} \Psi\right) \oplus\left(\bigoplus_{j \geqslant 1} b_{j}[0, j-1] \otimes \bigoplus_{k=0}^{o(\Psi)-1} \nu^{k} \Psi\right)
$$

then its standard form is equal to

$$
\bigoplus_{i \geqslant 1}^{o\left(\Psi_{0}-1\right)} \bigoplus_{k=0} a_{i, k}[0, i-1] \otimes \nu^{k} \Psi
$$

with $a_{i, k}=c_{i, k}+b_{i}$. Thus the decomposition of $(\Phi, U)$ as the direct sum of a cyclic and acyclic parameter is unique, and it is

$$
(\Phi, U)=(\Phi, U)_{\text {acyc }} \oplus(\Phi, U)_{\text {cyc }}
$$

We now define an injection $\mathrm{CV}$ of $\operatorname{Nilp}_{\mathrm{ss}}\left(\mathrm{D}, \overline{\mathbb{F}_{\ell}}\right)$ into $\left[\operatorname{Rep}_{\mathrm{ss}}\left(\mathrm{D}, \overline{\mathbb{F}_{\ell}}\right)\right]$, which is not the natural inclusion.

Definition 6.7. Take $(\Phi, U)=(\Phi, U)_{\mathrm{acyc}} \oplus(\Phi, U)_{\mathrm{cyc}} \in \operatorname{Nilp}_{\mathrm{ss}}\left(\mathrm{D}, \overline{\mathbb{F}_{\ell}}\right)$ supported on an irreducible line $\mathbb{Z}_{\Psi}$, and write

$$
(\Phi, U)_{\mathrm{cyc}}=\bigoplus_{j \geqslant 1} b_{j}[0, j-1] \otimes \bigoplus_{k=0}^{o(\Psi)-1} \nu^{k} \Psi .
$$

We set

$$
\mathrm{CV}\left((\Phi, U)_{\mathrm{cyc}}\right)=\bigoplus_{j \geqslant 1} b_{j}[0, j-1] \otimes \mathcal{C}\left(\mathbb{Z}_{\Psi}\right) \in\left[\operatorname{Rep}_{\mathrm{ss}}\left(\mathrm{D}, \overline{\mathbb{F}_{\ell}}\right)\right]
$$

and then

$$
\mathrm{CV}(\Phi, U)=(\Phi, U)_{\mathrm{acyc}} \oplus \mathrm{CV}\left((\Phi, U)_{\mathrm{cyc}}\right) \in\left[\operatorname{Rep}_{\mathrm{ss}}\left(\mathrm{D}, \overline{\mathbb{F}_{\ell}}\right)\right]
$$

Finally, if $(\Phi, U)=\bigoplus_{j}\left(\Phi\left(\mathbb{Z}_{\Psi_{j}}\right), U_{j}\right) \in \operatorname{Nilp}_{\mathrm{ss}}\left(\mathrm{D}, \overline{\mathbb{F}_{\ell}}\right)$ where $\Phi\left(\mathbb{Z}_{\Psi_{j}}\right)$ is supported on the irreducible line $\mathbb{Z}_{\Psi_{j}}$, and $\mathbb{Z}_{\Psi_{k}} \neq \mathbb{Z}_{\Psi_{l}}$ for $k \neq l$, we set

$$
\mathrm{CV}(\Phi, U)=\bigoplus_{j} \mathrm{CV}\left(\Phi\left(\mathbb{Z}_{\Psi_{j}}\right), U_{j}\right) .
$$

We have the following immediate lemma.

Lemma 6.8. The map CV $: \operatorname{Nilp}_{\mathrm{ss}}\left(\mathrm{D}, \overline{\mathbb{F}_{\ell}}\right) \rightarrow\left[\operatorname{Rep}_{\mathrm{ss}}\left(\mathrm{D}, \overline{\mathbb{F}_{\ell}}\right)\right]$ is injective.

Combining V and CV, we make the following definition: 
Definition 6.9. Let $\mathrm{C}: \operatorname{Irr}\left(G, \overline{\mathbb{F}_{\ell}}\right) \rightarrow\left[\operatorname{Rep}_{\mathrm{ss}}\left(\mathrm{D}, \overline{\mathbb{F}_{\ell}}\right)\right]$ denote the injection defined by

$$
\mathrm{C}(\pi)=\mathrm{CV}(\mathrm{V}(\pi))
$$

for $\pi \in \operatorname{Irr}(G)$.

We do the C-correspondence version of Example 6.5. We use the notation

$$
\mathcal{C}_{k}\left(\mathbb{Z}_{\Psi}\right):=\ell^{k} \mathcal{C}\left(\mathbb{Z}_{\Psi}\right)
$$

for $\Psi \in \operatorname{Irr}\left(\mathrm{W}_{F}, \overline{\mathbb{F}_{\ell}}\right)$ (in particular $\mathcal{C}_{0}\left(\mathbb{Z}_{\Psi}\right)=\mathcal{C}\left(\mathbb{Z}_{\Psi}\right)$ ).

Example 6.10. (1) If $\pi \in \operatorname{Irr}_{\mathrm{sc}}(G)$ and $\mathrm{V}(\pi)=\Psi$, then

$$
\mathrm{C}(\pi)= \begin{cases}\Psi & \text { if } \pi \text { is banal, } \\ \mathcal{C}\left(\mathbb{Z}_{\Psi}\right)=\mathcal{C}(\{\Psi\}) & \text { if } \pi \text { is non-banal. }\end{cases}
$$

(2) If $\rho=\operatorname{St}_{k}\left(\rho_{0}\right)$ is cuspidal non-supercuspidal with $\rho_{0} \in \operatorname{Irr}_{\mathrm{sc}}\left(G, \overline{\mathbb{F}_{\ell}}\right)$ and $\Psi_{0}=\mathrm{V}\left(\rho_{0}\right) \in$ $\operatorname{Irr}\left(\mathrm{W}_{F}, \overline{\mathbb{F}_{\ell}}\right)$, then

$$
\mathrm{C}(\rho)=\mathcal{C}_{k}\left(\mathbb{Z}_{\Psi_{0}}\right)
$$

(3) If $\rho$ is supercuspidal, and $\Psi=\mathrm{V}(\rho) \in \operatorname{Irr}\left(\mathrm{W}_{F}, \overline{\mathbb{F}_{\ell}}\right)$, then

$$
\mathrm{C}\left(\mathrm{L}\left([a, b]_{\rho}\right)\right)= \begin{cases}{[a, b] \otimes \Psi} & \text { if } \rho \text { is banal, } \\ {[a, b] \otimes \mathcal{C}\left(\mathbb{Z}_{\Psi}\right)} & \text { if } \rho \text { is non-banal. }\end{cases}
$$

More generally, for $k \geqslant 0$,

$$
\mathrm{C}\left(\mathrm{L}\left([a, b]_{\mathrm{St}_{k}(\rho)}\right)=[a, b] \otimes \mathcal{C}_{k}\left(\mathbb{Z}_{\Psi}\right)\right.
$$

(4) Take $\pi=\pi_{\mathrm{b}} \times \pi_{\text {tnb }} \in \operatorname{Irr}_{\text {gen }}\left(G, \overline{\mathbb{F}_{\ell}}\right)$, supported on the supercuspidal line $\mathbb{Z}_{\rho}$ with $\mathrm{V}(\rho)=\Psi$. Write

$$
\pi_{\mathrm{b}}=\prod_{i \geqslant 1} \prod_{k=0}^{o(\Psi)-1} \mathrm{~L}\left([0, i-1]_{\nu^{k}}\right)^{c_{i, k}}
$$

where the occuring segments are unlinked, hence in particular for each fixed $i$, there is a $k$ such that $c_{i, k}=0$. Write

$$
\pi_{\mathrm{tnb}}=\prod_{k \geqslant 0} \mathrm{~L}\left(\left[0, a_{k}-1\right]_{\mathrm{St}_{k}(\rho)}\right)
$$

with $0 \leqslant a_{k}<\ell$. Then

$$
\mathrm{V}(\pi)_{\text {acyc }}=\mathrm{V}\left(\pi_{b}\right) \text {, and } \mathrm{V}(\pi)_{\mathrm{cyc}}=\mathrm{V}\left(\pi_{\mathrm{tnb}}\right) .
$$

Hence

$$
\mathrm{C}(\pi)=\mathrm{C}\left(\pi_{\mathrm{b}}\right) \oplus \mathrm{C}\left(\pi_{\mathrm{tnb}}\right)
$$

with

$$
\begin{aligned}
\mathrm{C}\left(\pi_{\mathrm{b}}\right) & =\bigoplus_{i \geqslant 1} \bigoplus_{k=0}^{o(\Psi)-1} c_{i, k}[0, i-1] \otimes \nu^{k} \Psi \\
\mathrm{C}\left(\pi_{\mathrm{tnb}}\right) & =\bigoplus_{j \geqslant 0}\left[0, a_{j}-1\right] \otimes \mathcal{C}_{j}\left(\mathbb{Z}_{\Psi}\right) .
\end{aligned}
$$




\subsection{Preservation of local constants}

It follows immediately from its definition that CV commutes with taking duals, direct sums, twisting by characters, and does not change the determinant. Hence the C-correspondence shares with the V-correspondence the properties of Lemma 6.4.

Lemma 6.11. Let $\pi \in \operatorname{Irr}\left(G, \overline{\mathbb{F}_{\ell}}\right)$.

(1) For all characters $\chi: \mathrm{GL}_{n}(F) \rightarrow{\overline{\mathbb{F}_{\ell}}}^{\times}$,

$$
\mathrm{C}(\chi \pi)=\chi \mathrm{C}(\pi)
$$

(2) Letting $c_{\pi}$ denote the central character of $\pi$, then using local class field theory

$$
c_{\pi}=\operatorname{det}(\mathrm{C}(\pi)) .
$$

(3) We have

$$
\mathrm{C}\left(\pi^{\vee}\right)=\mathrm{C}(\pi)^{\vee}
$$

(4) For $\pi^{\prime} \in \operatorname{Irr}\left(G, \overline{\mathbb{F}_{\ell}}\right)$ such that $\pi \times \pi^{\prime}$ is irreducible, we have $\mathrm{C}\left(\pi \times \pi^{\prime}\right)=\mathrm{C}(\pi) \oplus \mathrm{C}(\pi)$.

(5) Moreover if $\pi=\pi\left(\mathbb{Z}_{\rho_{i}}\right) \times \cdots \times \pi\left(\mathbb{Z}_{\rho_{r}}\right)$ with $\pi\left(\mathbb{Z}_{\rho_{i}}\right)$ supported on the supercuspidal line $\mathbb{Z}_{\rho_{i}}$ and $\mathbb{Z}_{\rho_{i}} \neq \mathbb{Z}_{\rho_{j}}$ for $i \neq j$, then

$$
\mathrm{C}(\pi)=\bigoplus_{i=1}^{r} \mathrm{C}\left(\pi\left(\mathbb{Z}_{\rho_{i}}\right)\right)
$$

Hence for the moment we have not lost anything in introducing $\mathrm{C}$, except the (not so direct) compatibility with reduction modulo $\ell$ of the $\mathrm{V}$-correspondence. Of course, we can keep track of this property as the map CV is injective. On the other hand we have not gained anything yet. However there is one important property that the $\mathrm{V}$ correspondence does not share with the LLC, which is the preservation of local constants. For the last sentence to make sense, one must have a definition of local constants for elements in $\operatorname{Irr}\left(G, \overline{\mathbb{F}_{\ell}}\right)$. Indeed there is one: for standard local constants, they have been defined in [13] à la Godement-Jacquet, and for local constants of pairs, they have been defined in [12] for pairs of generic representations by the Rankin-Selberg method of Jacquet, Piatetski-Shapiro and Shalika. It should be true that $L^{\mathrm{RS}}(X, \pi, \mathbf{1})=L^{\mathrm{GJ}}(X, \pi)$, and similarly for $\gamma$ - and $\epsilon$-factors for $\pi \in \operatorname{Irr}_{\text {gen }}(G)$, but we did not check this. In what follows we will only consider the Rankin-Selberg local constants defined in [12], and we will drop the RS exponent. We claim that $\mathrm{C}$ preserves local constants of pairs, whereas $\mathrm{V}$ does not. Let us give a basic example where we consider the $L$-factor only.

Example 6.12. Consider the cuspidal representation $\rho=\operatorname{St}(o(\nu), \mathbf{1})$ of $\mathrm{G}_{n}$ for $n=o(\nu)$, then

$$
\mathrm{V}(\rho)=\bigoplus_{k=0}^{o(\nu)-1} \nu^{k}, \quad \text { whereas } \quad \mathrm{C}(\rho)=\mathcal{C}\left(\mathbb{Z}_{\mathbf{1}}\right) .
$$

According to [12, Theorem 4.9], we have

$$
L(X, \pi)=L(X, \pi, \mathbf{1})=1 .
$$

By definition of the Deligne $L$-factor, we also have

$$
L\left(X, \mathcal{C}\left(\mathbb{Z}_{\mathbf{1}}\right)\right)=1
$$

because the Deligne operator associated to $\mathcal{C}\left(\mathbb{Z}_{\mathbf{1}}\right)$ is bijective. However,

$$
L\left(X, \bigoplus_{k=0}^{o(\nu)-1} \nu^{k}\right)=\prod_{k=0}^{o(\nu)-1} \frac{1}{1-q^{-k} X} .
$$


We can finally prove the central result of this paper. We fix a nontrivial character $\psi: F \rightarrow{\overline{\mathbb{F}_{\ell}}}^{\times}$ and $\tilde{\psi}: F \rightarrow \overline{\mathbb{Z}}_{\ell} \times$ a lift of $\psi$.

Theorem 6.13. For $\pi, \pi^{\prime} \in \operatorname{Irr}_{\text {gen }}\left(G, \overline{\mathbb{F}_{\ell}}\right)$, we have

$$
\begin{aligned}
L\left(X, \mathrm{C}(\pi) \otimes_{\mathrm{ss}} \mathrm{C}\left(\pi^{\prime}\right)\right) & =L\left(X, \pi, \pi^{\prime}\right), \\
\gamma\left(X, \mathrm{C}(\pi) \otimes_{\mathrm{ss}} \mathrm{C}\left(\pi^{\prime}\right), \psi\right) & =\gamma\left(X, \pi, \pi^{\prime}, \psi\right), \\
\epsilon\left(X, \mathrm{C}(\pi) \otimes_{\mathrm{ss}} \mathrm{C}\left(\pi^{\prime}\right), \psi\right) & =\epsilon\left(X, \pi, \pi^{\prime}, \psi\right) .
\end{aligned}
$$

Proof. Observe that the equality for $\epsilon$-factors follows from the first two, by definition. Let's prove the statement for $\gamma$-factors first. Let $\pi$ and $\pi^{\prime}$ belong to $\operatorname{Irr}_{\text {gen }}(G)$ and choose $\tilde{\pi}$ and $\tilde{\pi}^{\prime}$ two $\ell$-adic generic representations such that $\pi=\mathrm{J}_{\ell}(\tilde{\pi})$ and $\pi^{\prime}=\mathrm{J}_{\ell}\left(\tilde{\pi^{\prime}}\right)$ (for example standard lifts as in [12, Definition 2.24]). Then according to [12, Theorem 3.13], one has

$$
\gamma\left(X, \pi, \pi^{\prime}, \psi\right)=r_{\ell}\left(\gamma\left(X, \tilde{\pi}, \tilde{\pi}^{\prime}, \tilde{\psi}\right)\right) .
$$

Set $\tilde{\Phi}$ and $\tilde{\Phi}^{\prime}$ be the semisimple representations of $\mathrm{W}_{F}$ corresponding to the supercuspidal support of $\tilde{\pi}$ and $\tilde{\pi}^{\prime}$ via $\operatorname{LLC}\left(\right.$ i.e. $\operatorname{LLC}(\tilde{\pi})=(\tilde{\Phi}, \star)$ and $\left.\operatorname{LLC}\left(\tilde{\pi}^{\prime}\right)=\left(\tilde{\Phi}^{\prime}, \star\right)\right)$. The LLC and the standard properties of $\gamma$-factors tell us that

$$
\gamma\left(X, \tilde{\pi}, \tilde{\pi}^{\prime}, \tilde{\psi}\right)=\gamma\left(X, \tilde{\Phi} \otimes \tilde{\Phi}^{\prime}, \tilde{\psi}\right) .
$$

Set $r_{\ell}(\tilde{\Phi})=\Phi$ and $r_{\ell}\left(\tilde{\Phi}^{\prime}\right)=\Phi^{\prime}$, hence $r_{\ell}\left(\tilde{\Phi} \otimes \tilde{\Phi}^{\prime}\right)=\Phi \otimes_{\mathrm{ss}} \Phi^{\prime}$. According to Theorem 6.3, or more simply [24, Theorem 1.6] which states that the semisimple LLC commutes with reduction modulo $\ell$, we deduce that $\Phi$ corresponds to the supercuspidal support of $\pi$, whereas $\Phi^{\prime}$ corresponds to the supercuspidal support of $\pi^{\prime}$, i.e. $\mathrm{C}(\pi)=[\Phi, \star]$ and $\mathrm{C}\left(\pi^{\prime}\right)=\left[\Phi^{\prime}, \star\right]$, so that

$$
\gamma\left(X, \mathrm{C}(\pi) \otimes_{\mathrm{ss}} \mathrm{C}\left(\pi^{\prime}\right), \psi\right)=\gamma\left(X, \Phi \otimes_{\mathrm{ss}} \Phi^{\prime}, \psi\right) .
$$

However

$$
\gamma\left(X, \Phi \otimes_{\mathrm{ss}} \Phi^{\prime}, \psi\right)=r_{\ell}\left(\gamma\left(X, \tilde{\Phi} \otimes \tilde{\Phi}^{\prime}, \tilde{\psi}\right)\right)
$$

according to Proposition 5.8. This ends the proof of the assertion on $\gamma$-factors.

It remains to prove that on $L$-factors. As before, we write $\pi=\pi_{\mathrm{b}} \times \pi_{\mathrm{tnb}}$ and $\pi^{\prime}=\pi_{\mathrm{b}}^{\prime} \times \pi_{\mathrm{tnb}}^{\prime}$ for their decompositions into banal and totally non-banal representations. By [12, Theorem 4.19] tells us that

$$
L\left(X, \pi, \pi^{\prime}\right)=L\left(X, \pi_{\mathrm{b}}, \pi_{\mathrm{b}}^{\prime}\right) .
$$

Set $\left[\Phi_{\mathrm{b}}, U_{\mathrm{b}}\right]=\mathrm{C}\left(\pi_{\mathrm{b}}\right),\left[\Phi_{\mathrm{tnb}}, U_{\mathrm{tnb}}\right]=\mathrm{C}\left(\pi_{\mathrm{tnb}}\right),\left[\Phi_{\mathrm{b}}^{\prime}, U_{\mathrm{b}}^{\prime}\right]=\mathrm{C}\left(\pi_{\mathrm{b}}^{\prime}\right),\left[\Phi_{\mathrm{tnb}}^{\prime}, U_{\mathrm{tnb}}^{\prime}\right]=\mathrm{C}\left(\pi_{\mathrm{tnb}}^{\prime}\right)$, in particular $U_{\mathrm{tnb}}$ and $U_{\mathrm{tnb}}^{\prime}$ are bijective whereas $U_{\mathrm{b}}$ and $U_{\mathrm{b}}^{\prime}$ are nilpotent as in Example 6.10 (4). One has

$\mathrm{C}(\pi) \otimes_{\mathrm{ss}} \mathrm{C}\left(\pi^{\prime}\right)=\left(\mathrm{C}\left(\pi_{\mathrm{b}}\right) \otimes_{\mathrm{ss}} \mathrm{C}\left(\pi_{\mathrm{b}}^{\prime}\right)\right) \oplus\left(\mathrm{C}\left(\pi_{\mathrm{b}}\right) \otimes_{\mathrm{ss}} \mathrm{C}\left(\pi_{\mathrm{tnb}}^{\prime}\right)\right) \oplus\left(\mathrm{C}\left(\pi_{\mathrm{tnb}}\right) \otimes_{\mathrm{ss}} \mathrm{C}\left(\pi_{\mathrm{b}}^{\prime}\right)\right) \oplus\left(\mathrm{C}\left(\pi_{\mathrm{tnb}}\right) \otimes_{\mathrm{ss}} \mathrm{C}\left(\pi_{\mathrm{tnb}}^{\prime}\right)\right)$.

If one writes any of the latter three direct summands under the form $[\Phi, U]$, then $U$ is bijective (see in particular Remark 4.40). This implies that

$$
L\left(X, \mathrm{C}(\pi) \otimes_{\mathrm{ss}} \mathrm{C}\left(\pi^{\prime}\right)\right)=L\left(X, \mathrm{C}\left(\pi_{\mathrm{b}}\right) \otimes_{\mathrm{ss}} \mathrm{C}\left(\pi_{\mathrm{b}}^{\prime}\right)\right) .
$$

Hence it remains to prove the equality:

$$
L\left(X, \mathrm{C}\left(\pi_{\mathrm{b}}\right) \otimes_{\mathrm{ss}} \mathrm{C}\left(\pi_{\mathrm{b}}^{\prime}\right)\right)=L\left(X, \pi_{\mathrm{b}}, \pi_{\mathrm{b}}^{\prime}\right)
$$

Now [12, Theorem 4.12 and Corollary 4.20], the multplicativity of Deligne $L$-factors with respect to direct sums, and the multiplicativity relation of Lemma 5.7 show that it is enough to prove it 
for $\pi_{b}=\rho$ and $\pi_{\mathrm{b}}^{\prime}=\rho^{\prime}$ banal supercuspidal representations. Take $\tilde{\rho}$ (resp. $\tilde{\rho}^{\prime}$ ) a cuspidal lift of $\rho\left(\right.$ resp. $\left.\rho^{\prime}\right)$, so that $\tilde{\Psi}=\operatorname{LLC}(\tilde{\rho})\left(\right.$ resp. $\left.\tilde{\Psi}^{\prime}=\operatorname{LLC}\left(\tilde{\rho}^{\prime}\right)\right)$ is an irreducible lift of $\Psi=\mathrm{C}(\rho)$ (resp. $\left.\Psi^{\prime}=\operatorname{LLC}\left(\rho^{\prime}\right)\right)$. Then

$$
L\left(X, \rho, \rho^{\prime}\right)=r_{\ell}\left(L\left(X, \tilde{\rho}, \tilde{\rho}^{\prime}\right)\right)
$$

by [12, Theorem 4.18], and

$$
L\left(X, \Psi \otimes_{\mathrm{ss}} \Psi^{\prime}\right)=r_{\ell}\left(L\left(X, \tilde{\Psi} \otimes \tilde{\Psi}^{\prime}\right)\right)
$$

thanks to Theorem 5.9. Moreover,

$$
L(X, \tilde{\rho}, \tilde{\rho})=L\left(X, \tilde{\Psi} \otimes \tilde{\Psi}^{\prime}\right)
$$

by the $\ell$-adic LLC, and we deduce the desired equality:

$$
L\left(X, \rho, \rho^{\prime}\right)=L\left(X, \Psi \otimes_{\mathrm{sS}} \Psi^{\prime}\right) .
$$

Remark 6.14. As we said we leave the equality $L^{\mathrm{GJ}}=L^{\mathrm{RS}}$ on generic representations for future work. We also believe $\mathrm{C}$ sends the Godement-Jacquet local constants of Mínguez on $\operatorname{Irr}\left(G, \overline{\mathbb{F}_{\ell}}\right)$ to the standard local constants on $\left[\operatorname{Rep}_{\mathrm{ss}}\left(\mathrm{D}, \overline{\mathbb{F}_{\ell}}\right)\right]$. For $G_{2}$ it can be checked as a consequence of the calculations carried out in [13, we leave the general case for a further investigation.

\section{References}

[1] Anne-Marie Aubert. Dualité dans le groupe de Grothendieck de la catégorie des représentations lisses de longueur finie d'un groupe réductif $p$-adique. Trans. Amer. Math. Soc., 347(6):2179-2189, 1995.

[2] I.N. Bernstein and A.V. Zelevinsky. Induced representations of reductive $p$-adic groups. I. Ann. Sci. École Norm. Sup. (4), 10(4):441-472, 1977.

[3] Colin J. Bushnell and Guy Henniart. The local Langlands conjecture for GL(2), volume 335 of Grundlehren der Mathematischen Wissenschaften /Fundamental Principles of Mathematical Sciences]. Springer-Verlag, Berlin, 2006.

[4] Colin J. Bushnell and Guy Henniart. Modular local Langlands correspondence for $\mathrm{GL}_{n}$. Int. Math. Res. Not. IMRN, (15):4124-4145, 2014.

[5] Jean-Francois Dat. Lefschetz operator and local Langlands mod $\ell$ : the regular case. Nagoya Math. J., 208:1-38, 2012.

[6] Jean-Francois Dat. Lefschetz operator and local Langlands modulo $\ell$ : the limit case. Algebra Number Theory, 8(3):729-766, 2014.

[7] P. Deligne. Formes modulaires et représentations de GL(2). In Modular functions of one variable, II (Proc. Internat. Summer School, Univ. Antwerp, Antwerp, 1972), pages 55-105. Lecture Notes in Math., Vol. 349, 1973.

[8] P. Deligne. Les constantes des équations fonctionnelles des fonctions L. In Modular functions of one variable, II (Proc. Internat. Summer School, Univ. Antwerp, Antwerp, 1972), pages 501-597. Lecture Notes in Math., Vol. 349, 1973.

[9] Matthew Emerton and David Helm. The local Langlands correspondence for $\mathrm{GL}_{n}$ in families. Ann. Sci. Éc. Norm. Supér. (4), 47(4):655-722, 2014. 
[10] D. Helm and G. Moss. Gamma factors in deformation rings. arXiv:1510.08743v2, 2017.

[11] David Helm. On the modified mod $p$ local Langlands correspondence for $\mathrm{GL}_{2}\left(\mathbb{Q}_{\ell}\right)$. Math. Res. Lett., 20(3):489-500, 2013.

[12] Robert Kurinczuk and Nadir Matringe. Rankin-Selberg local factors modulo $\ell$. Selecta Math. (N.S.), 23(1):767-811, 2017.

[13] Alberto Mínguez. Fonctions zêta $\ell$-modulaires. Nagoya Math. J., 208:39-65, 2012.

[14] Alberto Mínguez and Vincent Sécherre. Représentations lisses modulo $\ell$ de $\mathrm{GL}_{m}(D)$. Duke Math. J., 163(4):795-887, 2014.

[15] Alberto Mínguez and Vincent Sécherre. L'involution de Zelevinski modulo $\ell$. Represent. Theory, 19:236-262, 2015.

[16] David E. Rohrlich. Elliptic curves and the Weil-Deligne group. In Elliptic curves and related topics, volume 4 of CRM Proc. Lecture Notes, pages 125-157. Amer. Math. Soc., Providence, RI, 1994.

[17] Jean-Pierre Serre. Sur la semi-simplicité des produits tensoriels de représentations de groupes. Invent. Math., 116(1-3):513-530, 1994.

[18] J. T. Tate. Fourier analysis in number fields, and Hecke's zeta-functions. In Algebraic Number Theory (Proc. Instructional Conf., Brighton, 1965), pages 305-347. Thompson, Washington, D.C., 1967.

[19] Marie-France Vignéras. Représentations $\ell$-modulaires d'un groupe réductif p-adique avec $\ell \neq p$, volume 137 of Progress in Mathematics. Birkhäuser Boston Inc., Boston, MA, 1996.

[20] Marie-France Vignéras. à propos d'une conjecture de Langlands modulaire. In Finite reductive groups (Luminy, 1994), volume 141 of Progr. Math., pages 415-452. Birkhäuser Boston, Boston, MA, 1997.

[21] Marie-France Vignéras. Cohomology of sheaves on the building and $R$-representations. Invent. Math., 127(2):349-373, 1997.

[22] Marie-France Vignéras. Induced $R$-representations of $p$-adic reductive groups. Selecta Math. (N.S.), 4(4):549-623, 1998.

[23] Marie-France Vignéras. Congruences modulo $\ell$ between $\epsilon$ factors for cuspidal representations of GL(2). J. Théor. Nombres Bordeaux, 12(2):571-580, 2000. Colloque International de Théorie des Nombres (Talence, 1999).

[24] Marie-France Vignéras. Correspondance de Langlands semi-simple pour GL $(n, F)$ modulo $l \neq p$. Invent. Math., 144(1):177-223, 2001.

[25] Marie-France Vignéras. La conjecture de Langlands locale pour $\operatorname{GL}(n, F)$ modulo $l$ quand $l \neq p, l>n$. Ann. Sci. École Norm. Sup. (4), 34(6):789-816, 2001.

[26] Marie-France Vignéras. On highest Whittaker models and integral structures. In Contributions to automorphic forms, geometry, and number theory, pages 773-801. Johns Hopkins Univ. Press, Baltimore, MD, 2004.

[27] A.V. Zelevinsky. Induced representations of reductive $p$-adic groups. II. On irreducible representations of GL(n). Ann. Sci. École Norm. Sup. (4), 13(2):165-210, 1980. 